\title{
Solar Weather Event Modelling and Prediction
}

\author{
Mauro Messerotti • Francesca Zuccarello • Salvatore L. Guglielmino • \\ Volker Bothmer · Jean Lilensten · Giancarlo Noci • Marisa Storini · Henrik Lundstedt
}

Received: 17 March 2009 / Accepted: 1 September 2009 / Published online: 17 November 2009

(C) The Author(s) 2009

Abstract Key drivers of solar weather and mid-term solar weather are reviewed by considering a selection of relevant physics- and statistics-based scientific models as well as a

M. Messerotti $(\bowtie)$

INAF-Astronomical Observatory of Trieste, Loc. Basovizza 302, 34012 Trieste, Italy

e-mail: messerotti@oats.inaf.it

M. Messerotti

Department of Physics, University of Trieste, Via A. Valerio 2, 34127 Trieste, Italy

F. Zuccarello · S.L. Guglielmino

Department of Physics and Astronomy - Astrophysics Division, University of Catania, Via S. Sofia 78, 95123 Catania, Italy

F. Zuccarello

e-mail: fzu@oact.inaf.it

S.L. Guglielmino

e-mail: sgu@oact.inaf.it

V. Bothmer

Institute for Astrophysics, University of Göttingen, Friedrich-Hund-Platz 1, 37077 Göttingen, Germany e-mail: bothmer@astro.physik.uni-goettingen.de

J. Lilensten

Planetology Laboratory of Grenoble, Bâtiment D de Physique, B.P. 53, 38041 Grenoble Cedex 9, France e-mail: jean.lilensten@obs.ujf-grenoble.fr

G. Noci

INAF-Astrophysical Observatory of Arcetri, Largo Fermi 5, 50125 Firenze, Italy

e-mail: noci@arcetri.astro.it

M. Storini

INAF-IFSI Rome, Via del Fosso del Cavaliere 100, 00133 Rome, Italy

e-mail: marisa.storini@ifsi-roma.inaf.it

H. Lundstedt

Swedish Institute of Space Physics, Scheelev. 17, 22370 Lund, Sweden

e-mail: henrik@lund.irf.se 
selection of related prediction models, in order to provide an updated operational scenario for space weather applications. The characteristics and outcomes of the considered scientific and prediction models indicate that they only partially cope with the complex nature of solar activity for the lack of a detailed knowledge of the underlying physics. This is indicated by the fact that, on one hand, scientific models based on chaos theory and non-linear dynamics reproduce better the observed features, and, on the other hand, that prediction models based on statistics and artificial neural networks perform better. To date, the solar weather prediction success at most time and spatial scales is far from being satisfactory, but the forthcoming ground- and space-based high-resolution observations can add fundamental tiles to the modelling and predicting frameworks as well as the application of advanced mathematical approaches in the analysis of diachronic solar observations, that are a must to provide comprehensive and homogeneous data sets.

Keywords Solar weather - Solar activity - Space weather - Sun · Solar dynamo ·

Solar flare $\cdot$ Coronal mass ejection $\cdot$ Solar irradiance $\cdot$ Solar radio burst $\cdot$ Heliosphere $\cdot$ GPS

\section{Introduction}

The Sun is the primary source of space weather, as the physical state of interplanetary space is determined by the temporal and spatial variations of both the quasi-stationary and the transient particle and electromagnetic emissions originated by the star, as outlined in Fig. 1 and commented in next Sect. 2.

A cyclic but aperiodic behaviour, related to the evolution of inner plasma processes such as the dynamo and fluid motions, characterizes the appearance of physical drivers, as the localized magnetic fields in photospheric sunspots and in atmospheric layers. It, also, causes their instability, leading to energy release that results in a variety of phenomena affecting the solar-terrestrial environment. Solar activity, the complex variety of energetic phenomena, is therefore the manifestation of solar variability and is modulated accordingly at different time scales, ranging from a decade to centuries and millennia. Anyway, the quasi-periodic, multiscale behaviour of solar activity is suggestive of the chaotic nature of the Sun as a complex system, i.e., of the concurrent processes that originate activity phenomena (see Sect. 2.1). This intrinsic nature can prevent e.g. the prediction of most energetic events, as pointed out by Hudson (2007) who analysed the highly irregular pattern of occurrence over the last two solar cycles for major solar flares, $\gamma$-ray events, and solar energetic particle (SEP) fluences and suggested that such phenomena do not appear to follow the direct indices of solar magnetic activity, such as the sunspot number, being characterized by a non-Poisson occurrence distribution related to the physical nature of the inner processes originating active regions.

The time evolution of solar activity phenomena determines the physical state of the solar surface and atmospheric layers on short (solar weather) and long time scales (solar climate). Hence, in the framework of meteorology of space aimed at predicting space weather and space climate, the capability of predicting the primary drivers that determine solar weather and solar weather is relevant.

Many models have been proposed for the solar dynamo supposed to regulate the mid- to long-term evolution of solar activity, models also based on nonlinear dynamics and chaos theory that can explain many of the observed feature evolution, but a self-consistent comprehensive model still does not exist (see Sect. 3.2). 
Similarly, thanks to high time and space resolution observations both from ground and space, the physical knowledge of short-term solar processes and features improved significantly, which made possible the development of a series of refined physical models of many solar phenomena typologies (see a review of scientific models for the emergence of magnetic flux and active regions in Sect. 4.1.2, for solar flares in Sect. 4.2.1, for the solar wind in Sect. 4.4.3, for XUV-EUV irradiance in Sect. 4.6, for coronal mass ejections in Sect. 4.7.2). However, most theoretical models are limited to specific sub-categories of phenomena, verified through post-event analyses.

To set up a reliable prediction system for solar weather or, specifically, for the key categories of solar weather drivers, the modeler can rely on: (a) a complete knowledge of the physics of the related generation, evolution and decay processes, when (but seldom) available; (b) occurrence and evolution statistics derived from observations; (c) the existence of precursory phenomena.

As most physics-based models are limited to sub-categories of events and statistics-based models are descriptive of some peculiar aspects, hybrid prediction models are often used to exploit the characteristics of both approaches, in several cases refined by artificial intelligence techniques such as expert systems and artificial neural networks (see Sect. 2.3).

To provide an updated scenario about solar weather modelling and predicting as key tiles in space weather modelling and predicting, we summarize present knowledge about a selected set of fundamental solar drivers, reviewing the relevant scientific and prediction models. In particular, reviews of prediction models are reported respectively in Sect. 3.3 for solar cycles, in Sect. 4.1.3 for emergence of magnetic flux and active region formation, in Sect. 4.2.2 for solar flares, in Sect. 4.4.2 for solar wind, in Sect. 4.6 for XUV-EUV irradiance, and in Sect. 4.7.4 for coronal mass ejections.

The general scheme of the paper is as follows. In Sect. 2 we consider the physical nature of solar activity, its descriptors and sample advanced prediction methods by emphasizing the impact on life via its short- and long-term variations. Section 3 is focused on features related to long-term variations such as the solar dynamo, providing a review on models for the prediction of solar cycles. Section 4 deals with features related to short-term variations, such as sunspots, flares, coronal holes, solar wind, suprathermal solar particles, XUV-EUV emission, coronal mass ejections, geoeffective solar radio bursts, and the relevant scientific and prediction models. The conclusions are drawn in Sect. 5.

\section{Solar Activity and Solar Weather}

Solar activity is characterized by a large variety of interrelated plasma processes, involving the interplay between plasma flows and the magnetic field topology, that occur at different time scales by releasing energy spent for plasma heating, particle acceleration and emission of electromagnetic radiation outbursts.

Such phenomena have their deep roots inside the Sun, where the dynamo mechanism operates and fluid motions occur in a turbulent way, and extend to the outer layers of the Sun and to the interplanetary space.

Solar weather can be defined as the physical state of the solar plasma in the outer layers of the Sun as determined by the time and space evolution of solar activity phenomena on short time scales (milliseconds to months), whereas solar climate is relevant to longer time scales (years to billion years).

In turn, solar weather characterizes the physical state of the heliosphere, the region of space around the Sun permeated by the solar wind and confined by the interstellar wind, and 


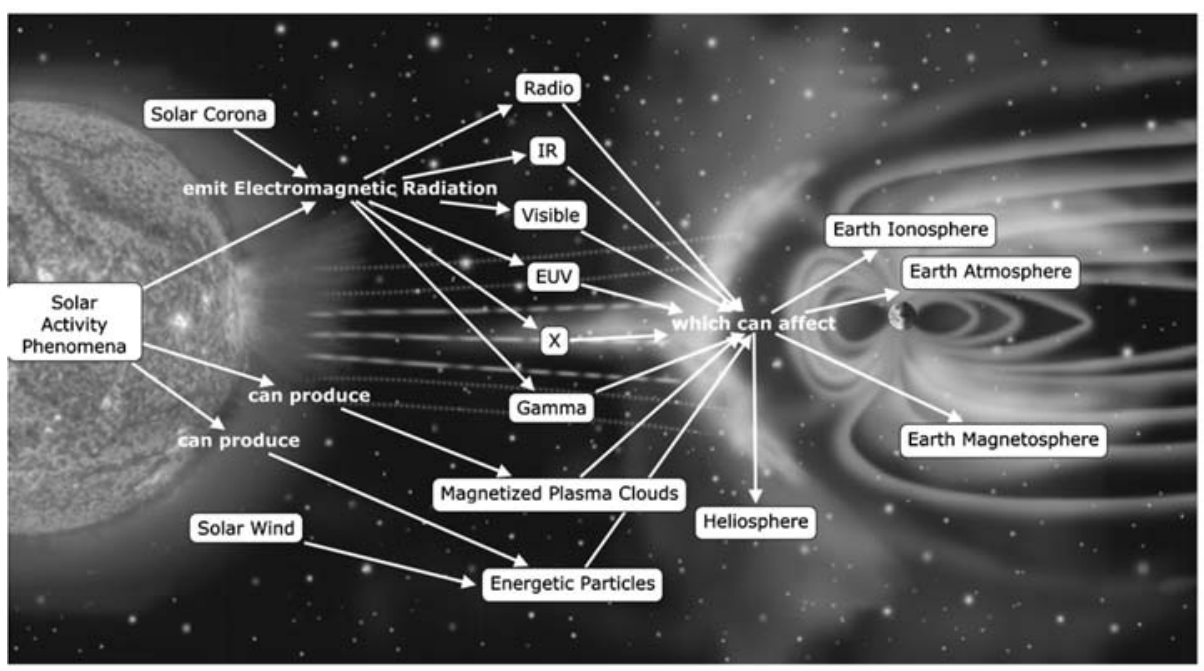

Fig. 1 Concept map that outlines solar weather drivers and their relationships to the solar-terrestrial environment. (Background photo courtesy of NASA/JPL/Caltech)

that of the regions of space surrounding planets and their magnetic fields, when existent, which is called geospace when referred to the Earth.

An outline of solar weather drivers and their interrelationships in the Sun-Earth environment is reported in Fig. 1 by a concept map (c-map; see e.g. Messerotti 2002, 2007, and references therein), generated by the c-map interactive development tool developed by IHMC (Institute for Human and Machine Cognition, USA; http://cmap.ihmc.us/).

$\mathrm{C}$-maps are a powerful tool for representing knowledge in graphical form: labelled polygons express concepts and labelled connecting lines state the relationships among concepts; concepts and relationships define the propositions which build up a semantic knowledge on a domain. Hence c-maps can be successfully used to code in graphical form the knowledge about a scientific domain by organizing the relevant information and terminology in a structured domain ontology. The advantage of such an approach is manifold, as it allows one: - to clearly identify concepts and relationships that becomes immediately evident from the cmap graph; - to define a science- (and, to some extent, not use-) based standard terminology; - to discover new knowledge by pointing out unexpected relationships. Moreover, c-maps are suitable to be coded in machine readable format other than in the human readable one, that is c-maps can constitute the knowledge skeleton for any ontology-based data management application (e.g., Messerotti 2002). Messerotti (2007) elaborated a preliminary version of a domain ontology for space weather and space climate entirely based on c-maps, and this approach has been successfully adopted in the framework of the past COST (EU Cooperation in Science and Technology) Action 724 "Developing the scientific basis for monitoring, modelling and predicting space weather" and the present COST Action ES0803 "Developing space weather products and services in Europe" (http://www.costes0803.noa.gr/).

To successfully predict solar weather implies the capability of predicting solar activity, which, in turn, implies a deep knowledge of the underpinning physics, i.e., the existence of a comprehensive and consistent set of physical models specific to each category of observed manifestations. A simplified scheme of solar activity drivers is outlined by the c-map in Fig. 2 (Messerotti 2007), which shows the complexity of the physical and observational scenario. 


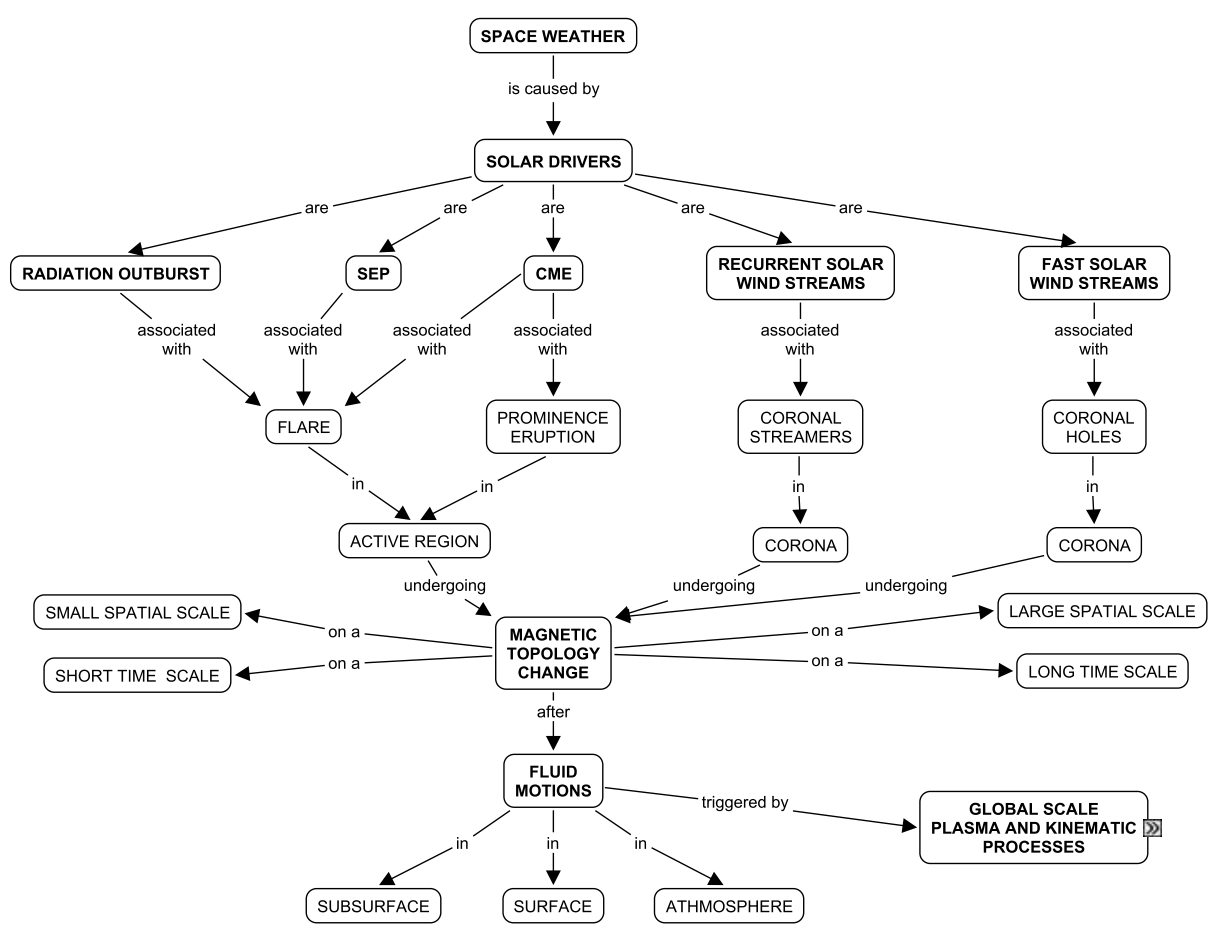

Fig. 2 Concept map that outlines solar activity drivers and their interrelationships (from Messerotti 2007). The radiation background component associated with active regions and plage evolution is not reported in this c-map

\subsection{Solar Activity as a Manifestation of a Chaotic Complex Plasma System}

The Sun can be considered as a complex plasma system subject to a set of physical processes spanning from the inner parts to the extended atmosphere, such as: (a) inner global fluid motions; (b) the generation of a global magnetic field; (c) differential and non-axisymmetric motions; (d) generation of localized magnetic fields; (e) external global fluid motions; (f) generation of a large-scale magnetic field.

Such processes occur at short to long time and spatial scales in plasma regions that are nonlinearly coupled via fluid motions and magnetic fields often in chaotic regimes, and are thought to be the drivers of solar activity. Hence, the variety of solar activity phenomena can be considered as the manifestation of coupled, multi-scale, chaotic processes (see e.g. Tél and Gruiz 2006; Regev 2006). Due to the intrinsic nature of a chaotic process, in principle the prediction of its time evolution is impossible. Notwithstanding, in specific cases some techniques based on nonlinear dynamics (see e.g. Sprott 2003) can be used provided that the underlying physics is reasonably well understood, which often is not the case. For instance, Sidorowich (1992) claimed that although frequently referred to as unpredictable deterministic behavior, chaotic systems can in fact be forecast over limited time scales, and elaborated on techniques for constructing predictive models for chaotic dynamics, including a variety of functional interpolation schemes and connectionist approaches to the problem. Lai et al. (1999) considered the modelling of deterministic chaotic systems, for which situations can arise where periodic orbits embedded in the chaotic set have distinct number of 
unstable directions and, as a consequence, no model of the system produces reasonably long trajectories that are realized by nature; despite of that they argue and present physical examples indicating that, in such a case, though the model is deterministic and low dimensional, statistical quantities can still be reliably computed. In a recent work, Woolley et al. (2009) proposed the use of an Artificial Neural Network (ANN) for modelling and prediction of time series generated by chaotic systems; the application to a chaotic data set obtained by solving the Lorenz equation led to a correlation of $94 \%$ and a negative Lyapunov exponent, indicating that the results obtained from ANN are in good agreement with the actual values. To date, such an approach seems to be the most promising and is widely used in prediction systems e.g. for sunspots and flares, as reported in Sects. 4.1.3 and 4.2.2.

The chaotic nature of the Sun as a complex physical system has been pointed out by various authors that focused on different processes from the core to the atmosphere, e.g.: - a proof of chaoticity based on the neutrino emission (Mandal and Raychaudhuri 2005); - the simulation of chaotically modulated stellar dynamos (Tobias et al. 1995); - the evidence of a chaotic behaviour in the solar dynamo from the variations of the solar magnetic field in the last 100 years (Lockwood et al. 1999); - the evidence of chaoticity from the analysis of the period and phase of the 88-year solar cycle (Feynman and Gabriel 2004); - the occurrence of grand minima/maxima driven by a stochastic/chaotic process (Usoskin et al. 2007); - a model of chaotic reconnection due to fast mixing of vortex-current filaments (Yatsuyanagi et al. 2000); - stochastic reconnection (Lazarian et al. 2004). Mundt et al. (1991) studied the variability of solar activity over long time scales, given semiquantitatively by measurements of sunspot numbers $R_{z}$, as a nonlinear dynamical system and found a positive Lyapunov exponent indicating that the solar activity variability described by $R_{z}$ can be described as a low dimension non-linear chaotic system of dimension 2.3. They then showed that predictions are only possible less than a couple of years ahead and compared the results to chaotic solar-dynamo models as a possible physically motivated source of the chaotic behavior. Chaos and intermittency in the solar cycle has been considered by Spiegel (2009), who pointed out that the number of spots on the Sun at any time varies in a cyclic, but aperiodic, manner; he analysed models with chaos and intermittency to reproduce the main qualitative aspects of the temporal variability, whereas the spatiotemporal variability requires a more complicated model and considered quite promising a description in terms of waves of excitation.

Therefore, an effective modelling of solar activity drivers has to consider the complexity of the physical system and incorporate it in the relevant model. This aspect makes even more complex the modellization and, probably, is indicative of the non-feasibility of a selfconsistent global model of solar activity, capable to successfully reproduce the evolution and coupling of solar drivers in the spatio-temporal domain as well as the generation and evolution of relevant solar weather events in a unified modelling framework. Advanced models have been developed for specific drivers and are quite promising as significant tiles in the development of effective predictive models suitable for space weather purposes, but in most cases they have been still science models and not operational models for space weather forecasting due to intrinsic limitations in the physics and to the highly demanding computing power required. In this context, we mention, e.g., the radiative magnetohydrodynamic 3D simulation of sunspot structure by Rempel et al. (2009) and the analysis of sunspots observations and simulations from small-scale inhomogeneities towards a global theory carried out by Schlichenmaier (2009) where he concludes that the understanding of the smallscales will be the key to understanding the global structure and the large-scale stability of sunspots. Difficulties and prospects in understanding the coronal magnetic field are reviewed by Cargill (2009), who considered the key observational inputs expected from new generation instruments like the Advanced Technology Solar Telescope (ATST; Keil et al. 2009) 
and the Frequency Agile Solar Radiotelescope (FASR; Bastian 2003), and states that global magnetic field models can provide information on the field in the corona, and towards the Earth through the solar wind, but major challenges for such modelling are the incorporation of small-scale plasma effects.

\subsection{Solar Activity Descriptors}

Solar activity is described by a set of descriptors, defined as either observable or observablederived entities and named indices or proxies when there is no direct relationship with the considered observable and they are inferred from other observables with a tight or a loose physical coupling with the considered one (see e.g. Tapping 2000; Messerotti 2001): A= $\mathbf{A}(\mathbf{s} ; t ; \mathbf{E})$, where $\mathbf{A} \in \mathbb{R}^{n}, \mathbf{s} \in \mathbb{R}^{3}$ is a spatial variable, $t \in \mathbb{R}^{1}$ is a time variable, and $\mathbf{E} \in \mathbb{R}^{n}$ is an energy variable.

An activity feature can be characterized by $n$ morphology descriptors $M_{i}=M_{i}(x, y, z)$ and magnetic ones $\mathcal{M}_{i}=\mathcal{M}_{i}(x, y, z)$ with $i \in[0, n-1]$, according to a classification based on a defined set of variation ranges.

The evolution of the activity feature is described by the time evolution of the relevant set of descriptors, $M_{i}=M_{i}(x, y, z ; t)$ and $\mathcal{M}_{i}=\mathcal{M}_{i}(x, y, z ; t)$ respectively.

The potentiality of an activity feature to generate or trigger geoeffective events (i.e., events that result in effects in geospace) can be estimated by the time evolution of the related descriptors, provided that an adequate physical model for the feature formation and evolution process is known. A deeper knowledge of the physical process together with a significant statistics based on observations can lead to the identification of a precursor state defined by specific values of the related descriptors for a specific activity feature when supposed to originate geoeffective events. The identification of a precursor or class of precursors helps in developing prediction techniques when dealing with solar activity features not adequately described by the associated descriptors.

As clearly pointed out in the following sections about key drivers of solar activity, global descriptors appear inadequate to provide a comprehensive scenario due to the chaotic nature of the underpinning processes which greatly expand the complexity of the behaviour of the Sun as a complex physical system. This, in turn, heavily biases the reliability of prediction systems. Hence more refined analyses and mathematical descriptions of the physics are needed to improve the understanding and, consequently, the prediction reliability.

\subsection{Advanced Analysis and Prediction Techniques}

To cope with the non-linearity and chaotic nature of solar magnetic activity, an approach based on intelligent hybrid systems proved to be very successful. In this section we outline the method conceived by Lundstedt (2006) as an introductory use case to the ones specific to the selected categories of solar activity features detailed in the following sections. In Sect. 3.3 predictions of the cycles are discussed and in Sect. 4.2.2 the use of solar activity indicators in flare prediction is considered.

In the model by Lundstedt, solar magnetic activity (SMA) is interpreted in terms of the interplay between the solar plasma flow, described by vector $\mathbf{V}$, and the solar magnetic field, described by vector $\mathbf{B}$. Hence SMA is described by using both mathematical and physical concepts. Such descriptions are integrated into a hybrid neural network as outlined in Fig. 3.

To illustrate the operational scenario, we present two examples of predictions based on observations of the plasma flow and the magnetic field in Sects. 2.3.1 and 2.3.2, respectively related to solar weather, i.e., short-term variations, and solar climate, i.e., long-term variations. Furthermore, in Sect. 2.3.3 we stress the need to remove the X-ray background in $\mathrm{X}$-ray irradiance data for a proper characterization of flares in operational models. 


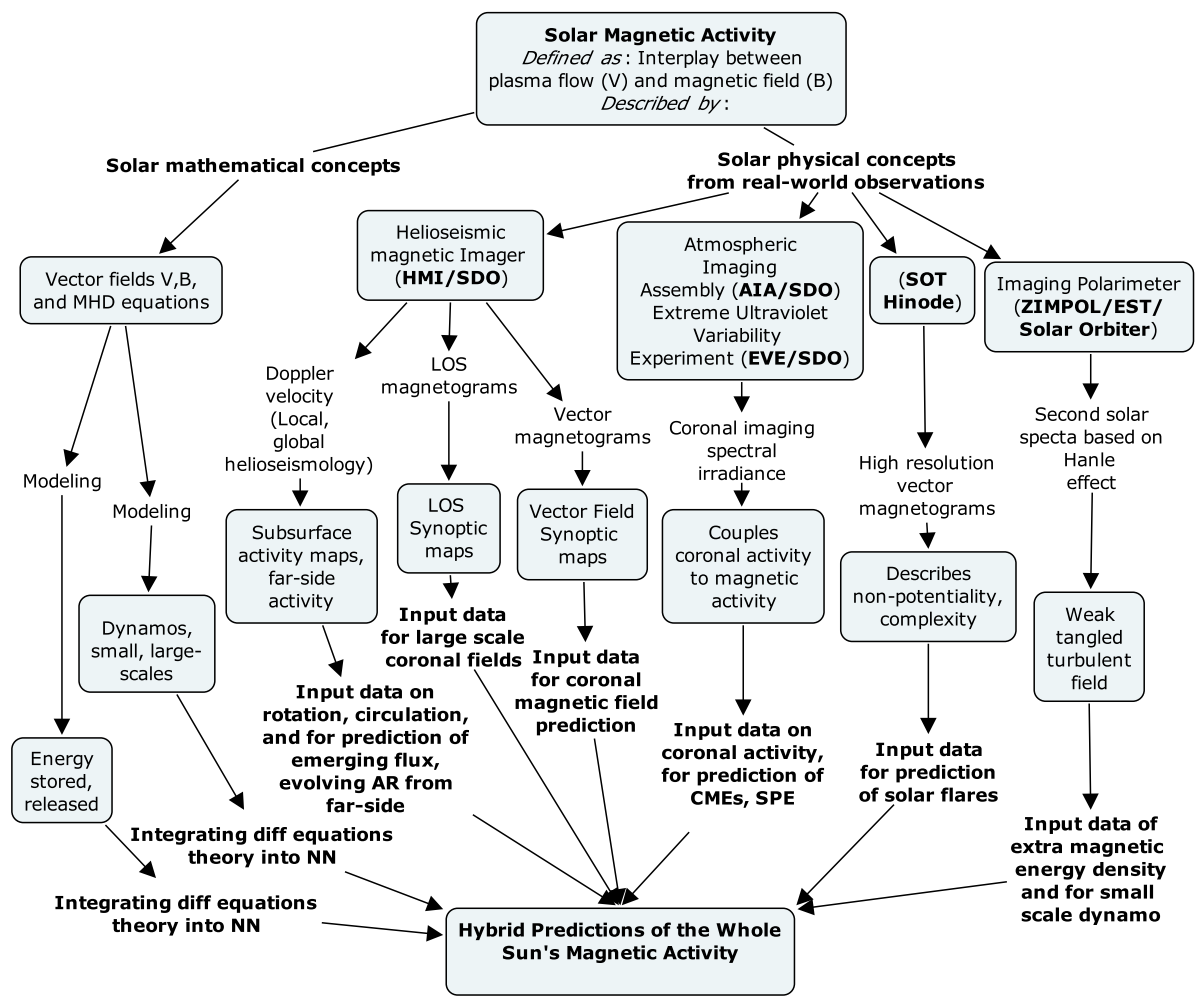

Fig. 3 Concept map outlining an advanced scheme for the prediction of Solar Magnetic Activity according to Lundstedt (2006)

\subsubsection{Predicting Solar Flares via Subsurface Flows Observations}

Jensen et al. (2004) pointed out a significant correlation between strong plasma downflows and high magnetic activity, indicated by strong solar flares (see Fig. 4).

A neural network was trained to predict an event of at least one major solar flare based on maps of subsurface flows and such predictions were quite promising (Fig. 4), despite the lack of a large input data set (Jensen et al. 2004).

A large data set and near-real-time maps, required for improving the prediction success, will become available from both the Global Oscillation Network Group (GONG) and, shortly, from the Solar Dynamics Observatory (SDO).

\subsubsection{Predicting Magnetic Activity Years Ahead}

Global patterns are visualized in solar synoptic maps relevant to e.g. sub-surface flows, photospheric and coronal magnetic fields.

Lundstedt et al. (2006) averaged longitudinally synoptic maps from Wilcox Solar Observatory (WSO) at Stanford covering three solar cycles from 1976 up to present. The averaged map is shown in Fig. 5, where global features can be identified like the variation of the Butterfly diagrams, the flux transport to the poles, and the asymmetry for both hemispheres.

Based on data from the longitudinally-averaged synoptic map, neural networks have been trained to predict the total magnetic flux Carrington rotations ahead. A correlation coefficient 


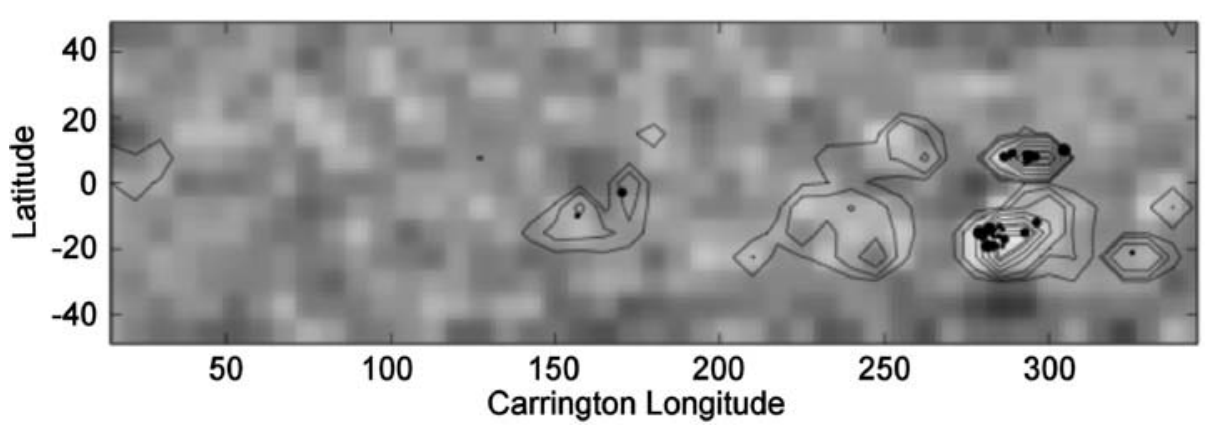

Fig. 4 Synoptic map for Carrington rotation 2009 (Halloween storm 2003) showing the divergence of the observed flows at a depth of $4.6 \mathrm{Mm}$. Bright regions represent inflow and dark ones outflow. The contour lines show the magnitude of magnetic field strength. The size of the dots indicate the flare $\mathrm{X}$-class: small $\rightarrow \mathrm{C}$, medium $\rightarrow \mathrm{M}$ and large $\rightarrow \mathrm{X}$ flares

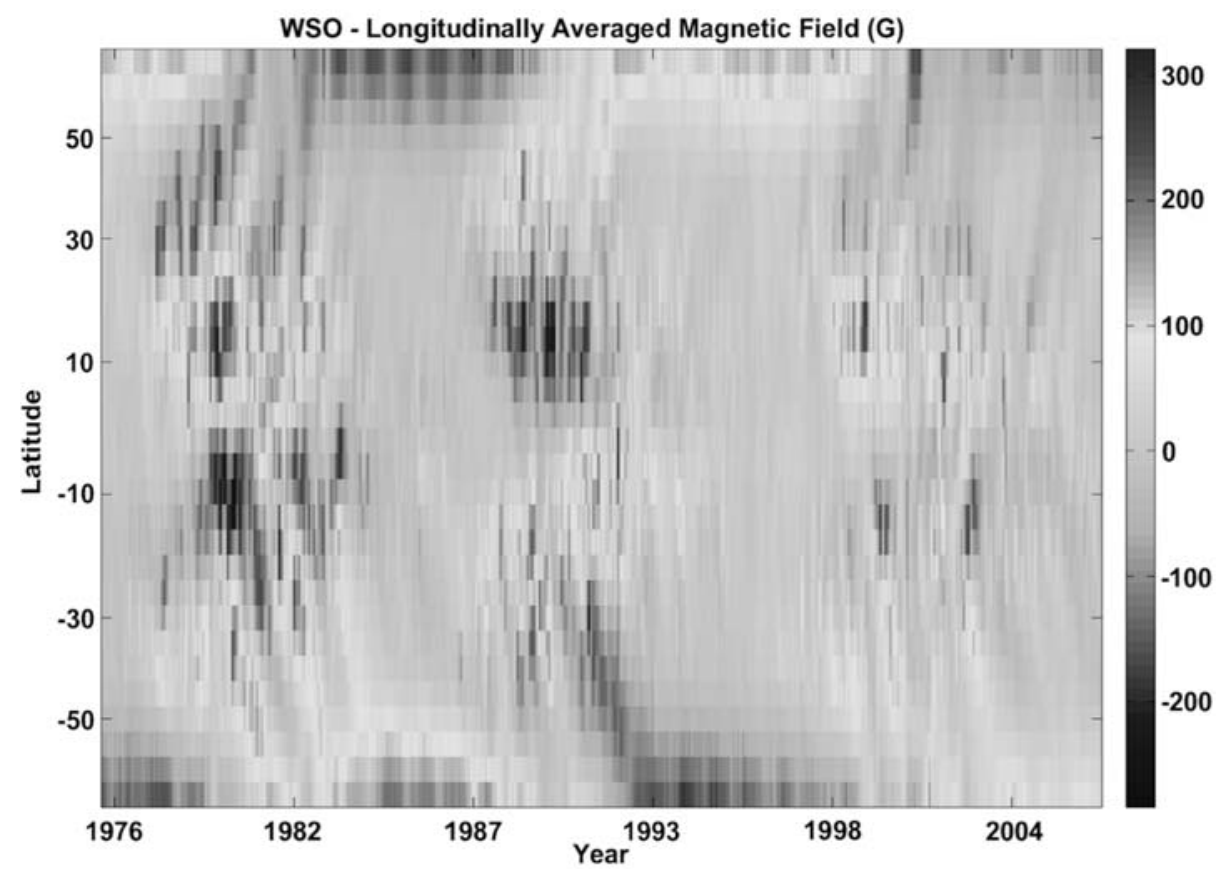

Fig. 5 Map of the longitudinally-averaged synoptic magnetic field (Lundstedt et al. 2006)

of 0.82 was reached between the predicted and observed values two years ahead (Lundstedt et al. 2006). Similar studies are planned using SDO data.

\subsubsection{X-ray Flare Characterization}

$\mathrm{X}$-ray background flux removal is a key aspect for the effective characterization of X-ray flares in GOES X-ray irradiance data (Tobiska and Bouwer 2004, 2005, 2006). In fact, the long-lived X-ray background flux is originated by a number of coronal X-ray sources not 
related to the flare under consideration. As flare timings (initiation, timing of peak flux, magnitude of peak flux, and decay from peak flux to background levels) are defined from the X-ray irradiance curve, they can be strongly biased when the background flux is not adequately removed and this affects the effectiveness of operational models. To achieve this goal, the cited authors proposed a method based on two indices respectively for the longlived component (background) and for the short-lived one (flare). The first index $\left(\mathrm{X}_{b 10}\right)$ is the $\log _{10}$ unitless number representing the lowest daily decile of the reported GOES XUV $0.1-0.8$ minutely data, which represents the $T \approx 10^{6} \mathrm{~K}$ coronal emission gradually evolving on active region time scales. The second one $\left(\mathrm{X}_{h f}\right)$ is the $\log _{10}$ unitless number representing the difference between the daily (previous running 24 hours) $\mathrm{X}_{b 10}$ background value that is created hourly and the median of the $\mathrm{XUV}_{0.1-0.8}$ measurements each hour, which provides a good estimate of $T \approx 10^{6} \mathrm{~K}$ or $T \approx 10^{7} \mathrm{~K}$ hot coronal flare activity.

\subsection{Solar Activity and Life}

In a multi-disciplinary approach, it is worthwhile mentioning that the study of solar weather and solar climate is not only relevant to set up, whenever possible, mitigation practices for everyday life at present time, but is also fundamental in understanding the suitability of a planet to favour life emergence and life persistence as considered in astrobiology.

The physical state of a planetary environment is determined by the physical state of the outer environment determined in turn by the central star, e.g. the Sun in the Solar System. In fact, on a short to long time scale solar activity perturbs the heliosphere by radiation outbursts and energetic particles. Such perturbations, which in turn characterize space weather and space climate, strongly bias the habitability of a planet, as they can act as life inhibitors or catalysts (see e.g. Messerotti 2005, 2006).

Constraints about the origin and evolution of life on Earth are set by the evolution of space weather and space climate, i.e. by the evolution of the Sun as a star via the evolution of solar weather and solar climate, which can be derived by analysing the imprints of solar energetic particles during the first billion years after the formation of the Sun (see e.g. Messerotti and Chela-Flores 2007, 2009, and references therein). In particular, Chela-Flores et al. (2008) carried an extensive review on astronomical and astrobiological imprints on the fossil records, by considering space and solar palaeoclimate conditions inferred from solar weather terrestrial proxies.

\section{Features and Models Relevant to Long-term Variations}

The short-term solar activity, and hence solar weather, is modulated on mid- and long-terms.

The mid-term modulation exhibits a periodicity of about 11 years and is the most wellknown. However, long-term modulation of periodicities of about 90 years (Gleissberg cycle), 200 years (DeVries cycle) and about 2300 years (Hallstatt cycle) have been identified as well.

Moreover, Usoskin et al. (2007) performed a reconstruction of sunspot numbers stretching over multiple millennia from ${ }^{14} \mathrm{C}$ data by means of a physics-based model, using an updated model of the evolution of the solar open magnetic flux. This analysis indicates that the occurrence of grand minima/maxima is not driven by a long-term cyclic variability, but by a stochastic/chaotic process related to the dynamo process evolution. 


\subsection{Descriptors of the Mid- and Long-term Solar Activity}

The most often used descriptor of the mid-term solar activity is the sunspot number $R_{z}$, defined as $R_{z}=k(10 g+s)$, where $g$ is the number of sunspot groups, $\mathrm{s}$ the number of individual sunspots, and $k$ a correction factor depending on the observer. The sunspot group number $R_{g}$ is defined as $R_{g}=\left(\frac{12.08}{n} \sum k G\right)$ (Hoyt and Schatten 1998), where $n$ is the number of observers, $G$ the number of sunspot groups and $k$ a correction factor. The group sunspot number is representative of the manifestation of an East-West magnet produced by the stretching of an initial poloidal North-South field under the effect of a non-uniform rotation.

During each cycle, the mean latitude of emergence of sunspots decreases as the cycle evolves as represented by the butterfly diagram, which shows that at the beginning of a cycle sunspots appear at high latitudes, between $25^{\circ}$ and $45^{\circ}$, while at the end of a cycle they appear at low latitudes, between $0^{\circ}$ and $20^{\circ}$.

The sunspot number is also used as an indicator of long-term Solar Magnetic Activity, but, at most, it covers only 23 sunspot cycles. These cycles largely differ both in amplitude and length. During the so-called Maunder Minimum (MM) 1645-1715, almost no sunspots were observed. Yet, Lundstedt et al. (2006) demonstrated via a refined wavelet analysis that the ${ }^{14} \mathrm{C}$ production showed about 11 -year variations during this period (Fig. 6)

A non-linear, chaotic, dynamical system shows many similarities with the variations of solar activity as indicated by the sunspot number. Tobias et al. (1995) managed to reproduce many of such features by means of a low order differential system based on Lorenz equations to simulate chaotically modulated solar dynamos (see also Usoskin et al. 2007).

\subsection{The Solar Dynamo}

Mathematically, the dynamo problem consists of trying to find solutions of MHD equations with a non-decaying total magnetic energy. The relevant set of equations (induction, momentum, continuity, energy and gas law equation) is given below.

$$
\begin{aligned}
\frac{\partial \mathbf{B}}{\partial t} & =\nabla \times(\mathbf{u} \times \mathbf{B})+\eta \nabla^{2} \mathbf{B} \\
\rho\left(\frac{\partial \mathbf{u}}{\partial t}+\mathbf{u} \cdot \nabla \mathbf{u}\right) & =-\nabla p+\mathbf{j} \times \mathbf{B}+\rho \mathbf{g}+\mathbf{F}_{\text {viscous }}+\mathbf{F}_{\text {other }} \\
\frac{\partial \rho}{\partial t}+\nabla \cdot(\rho \mathbf{u}) & =0 \\
\frac{D\left(p \rho^{\gamma}\right)}{D t} & =\text { loss terms } \\
p & =R \rho T
\end{aligned}
$$

and

$$
(\nabla \cdot \mathbf{B}=0) .
$$

The solar dynamo (Tobias 2002; Ossendrijver 2003; Charbonneau and Living 2005; Weiss and Thompson 2009) is an enormous challenge both to theoreticians and observers. Recent high-resolution observations of the solar surface reveal a magnetic field with a complex, hierarchical structure and widely different length scales. The dynamo theory provides 


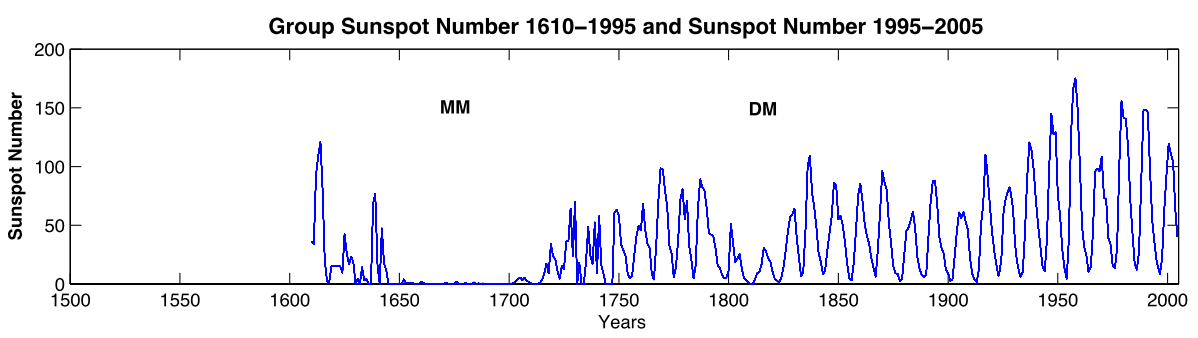

Annual C14 Production rate 1500-1950

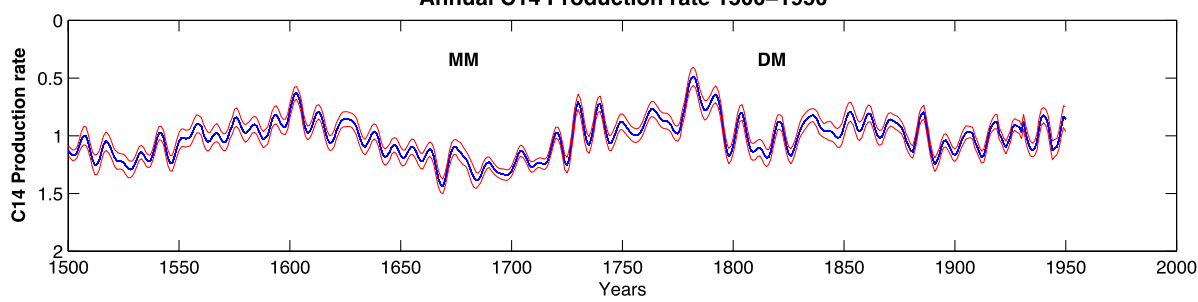

C14 Production rate $-9950(11500 \mathrm{BP})-1950(0)$

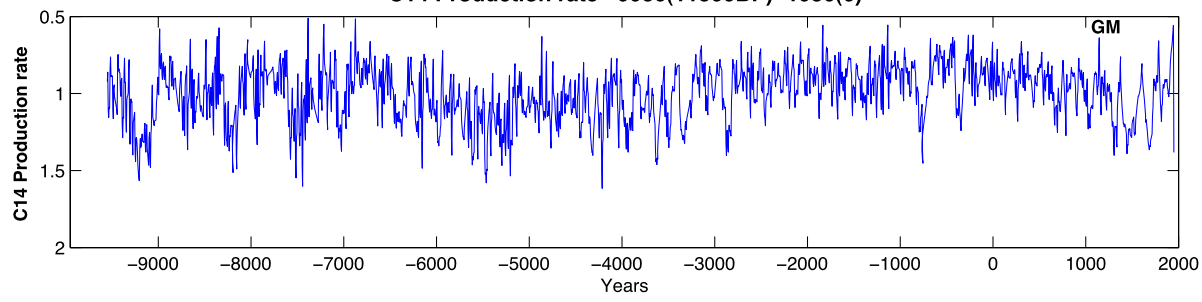

Fig. 6 The group sunspot number $R_{G}$ 1610-1995 and the sunspot number $R_{z}$ 1995-2005 (upper panel), the ${ }^{14} \mathrm{C}$ production rate $1500-1950$ (middle panel), and the ${ }^{14} \mathrm{C}$ production rate $9950 \mathrm{BC}-1950 \mathrm{AD}$ (lower panel). (MM-Maunder Minimum 1645-1715; DM-Dalton Minimum 1790-1820; GM-Grand Maximum 1100-1250) (from Lundstedt et al. 2006)

all the necessary ingredients to explain the magnetic phenomena, including the $\alpha$-effect, magnetic field amplification by differential rotation, turbulent diffusion, magnetic pumping, flux storage, magnetic buoyancy, stochastic variations and nonlinear dynamics. By means of advances in helioseismology, observations of stellar magnetic fields and computational facilities, significant progresses have been made in the understanding of various aspects such as the role of the tachocline (the region between the inner radiative shell and the outer convective layer which plays a fundamental role in the dynamo mechanism), convective plumes and magnetic helicity conservation. Recent simulations have also advanced our knowledge about the nature of the turbulent flow in the convective zone (Nordlund et al. 2009). The simulations by Stein (Nordlund et al. 2009) show both that individual packets of material seem to dive all the way to the base of the convection zone from the intergranular lanes, and also a coalescence of small-scale convection flows with depth, causing decoherence of the granular pattern with depth.

Parker (1955) developed the first solar-dynamo models more than half a century ago. Since then they evolved to accommodate observational constraints.

The large-scale solar dynamo involves three basic processes: (1) the generation of toroidal fields by shearing the pre-existing poloidal fields by differential rotation (the $\Omega$ effect); (2) re-generation of poloidal fields by lifting and twisting the toroidal flux tubes (the $\alpha$-effect); (3) flux transport by meridional circulation. The third mechanism was in- 
troduced in order to explain the weak, diffuse fields outside the sunspots belts. Incorporating a meridional circulation in each hemisphere, flux-transport dynamos have been constructed capable of reproducing many observed features, such as: (a) the full cycle period of $\sim 22$ years; (b) the field strength $(\sim 100 \mathrm{kG})$ of the toroidal field at the bottom of the convection zone; (c) the $\sim 10 \mathrm{G}$ polar field; (d) the magnetic coupling between the North and South hemispheres, in agreement with Hale's polarity rule (Dikpati 2004). But it should be kept in mind that the meridional circulation is derived from surface observations of the poleward flow at surface and an unobserved equatorwards flow at the base of the convective zone, based on mass conservation. Further uncertainty is also the assumption of a single cell for the meridional flow.

Small-scale solar dynamos are suggested by observations of the intranetwork magnetic fields that reveal the existence of a background magnetic flux residing in small scales and characterized by mixed polarities, a seemingly random spatial distribution, and no solar cycle dependence.

Simon et al. (2001) demonstrated that flux probably is generated on multiple scales, of which only the largest are dependent on the solar cycle. Schrijver et al. (1997) have developed the notion of cross-scale "magnetochemistry", building the notion that a chaotic cascade is the magnetic driver of space weather. Parnell et al. (2009) have found a power law probability distribution function of solar magnetic features across all detectable flux scales, from the largest active regions to the smallest intergranular flux concentrations. This suggests that the entire dynamo is dominated by a single scale-invariant mechanism that might be quenched at large scales by the physical size of the Sun itself.

Many uncertainties and open issues about the dynamos however still exist (see, e.g., Parker 2009). The ones relevant to predictions of the solar cycles are discussed in the next section.

\subsection{Models for the Prediction of Solar Cycles: Application to Cycle 24}

NOAA (National Oceanic and Atmospheric Administration), NASA and ISES (International Space Environment Service) sponsored a panel, consisting of 11 participants, to try to reach a consensus on the next sunspot cycle, Cycle 24.

Pesnell (2008) carried out a comprehensive review of 54 different predictions obtained by applying techniques based on climatology (past behaviour), dynamo models, spectral analysis, neural networks, geomagnetic and solar precursor methods. As a reference, in Table 1 we report all the prediction models considered by Pesnell, grouped by category (and within each category ordered by reference date) to provide a scenario of the refinement evolution in this field.

As can be seen in Pesnell (2008) (see Table 1 and Fig. 1 in the original paper), the predictions for Cycle 24 range from very low to very high values for the maximum sunspot number $R_{24}$. In fact, Svalgaard et al. (2005) predicted e.g. Cycle 24 to be the weakest in 100 years $\left(R_{24}=70 \pm 2\right.$ ) based on the polar field strength, whereas Dikpati et al. (2006), on the other hand, predicted a strong Cycle $24\left(R_{24}=155-180\right)$ based on applying a dynamo model.

On April 25, 2007 the panel announced their first predictions: Solar Minimum will occur on March, 2008 ( \pm 6 months), which marks the end of Cycle 23 and the start of Cycle 24. The length of Cycle 23 will then be 11.75 years, i.e., longer than the average of 11 years.

Cycle 24 will peak at a sunspot number of $140( \pm 20)$ in October, 2011 or it will peak at a sunspot number of $90( \pm 10)$ in August, 2012. An average solar cycle peaks at 114 and therefore the next cycle will be neither extreme nor average. 


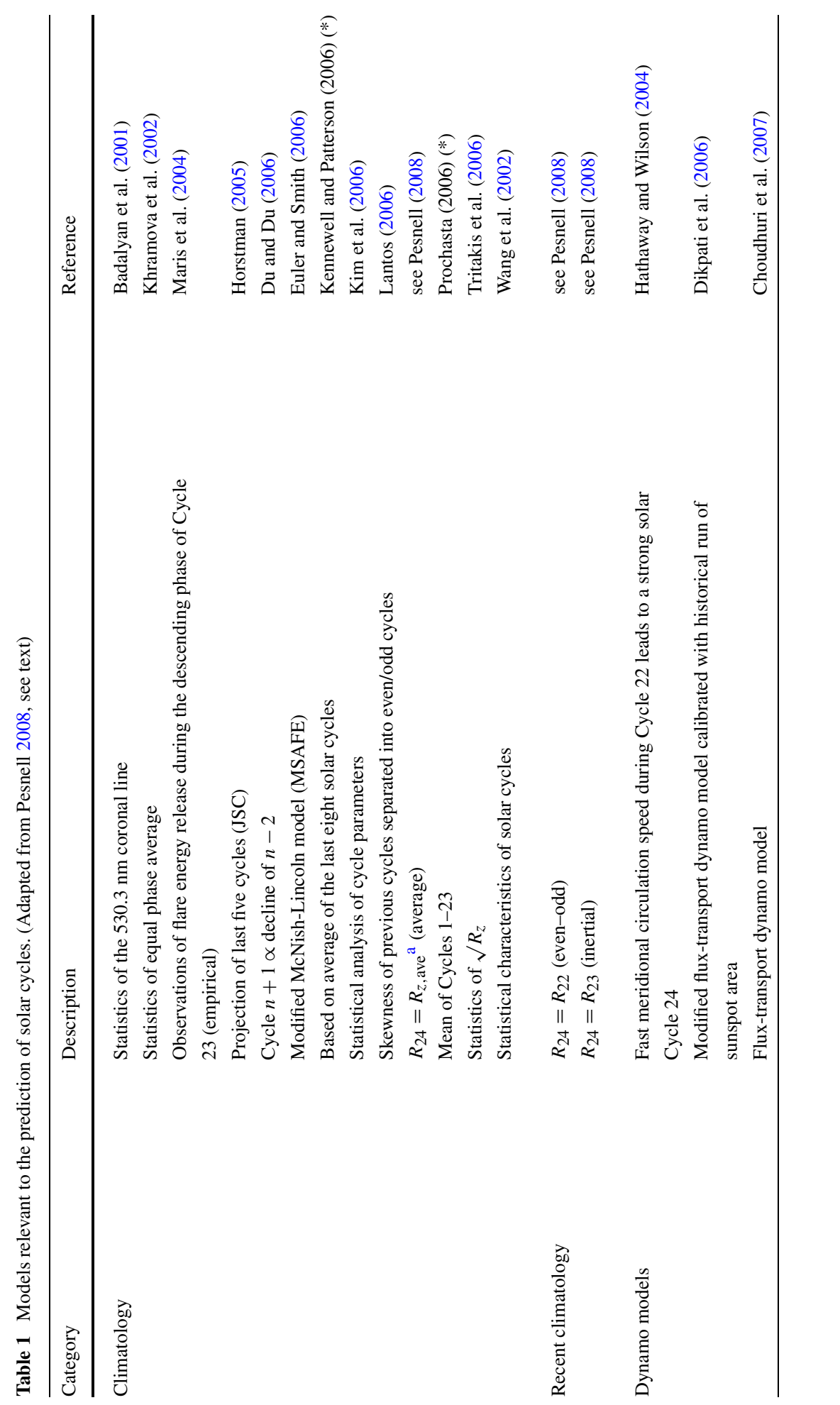




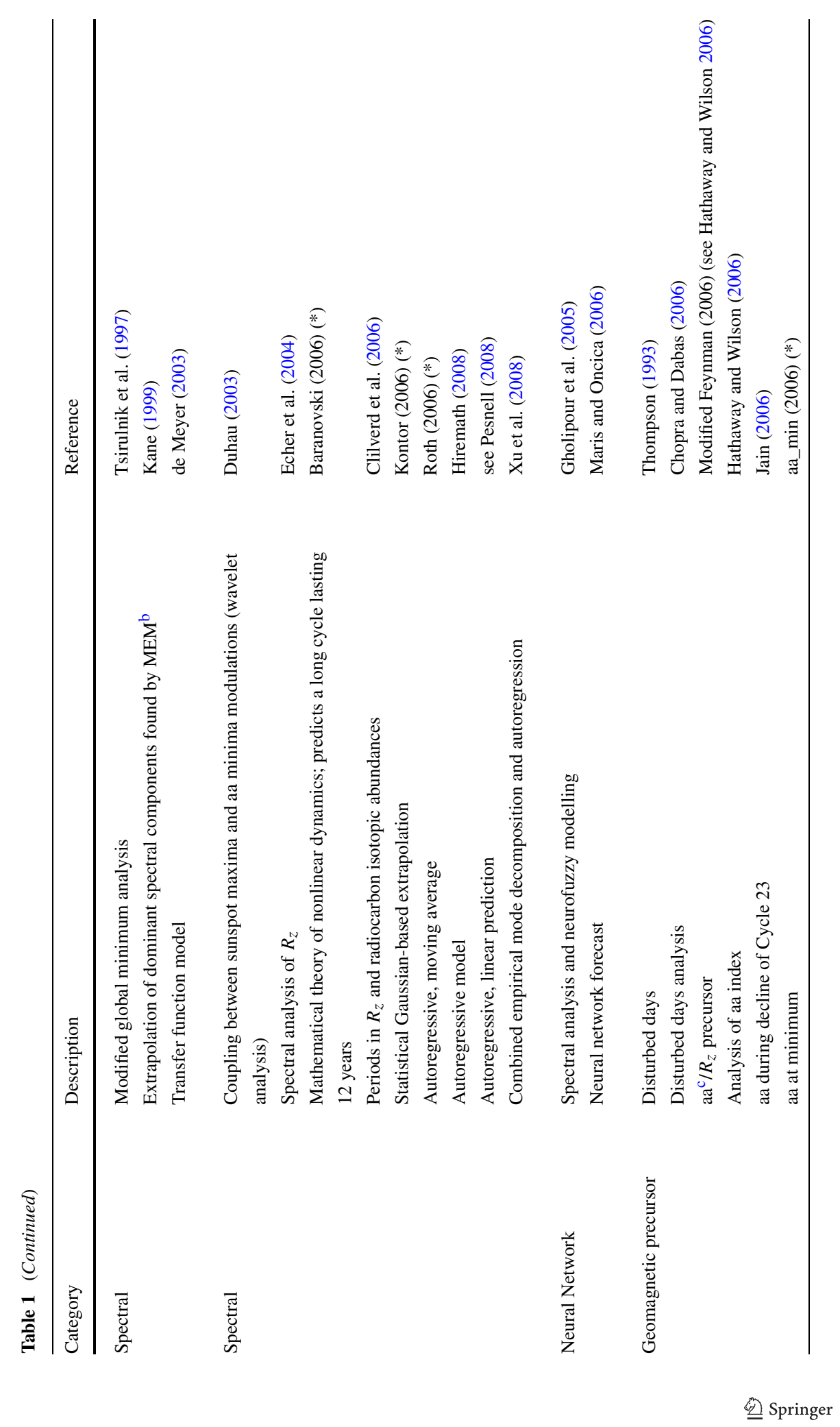




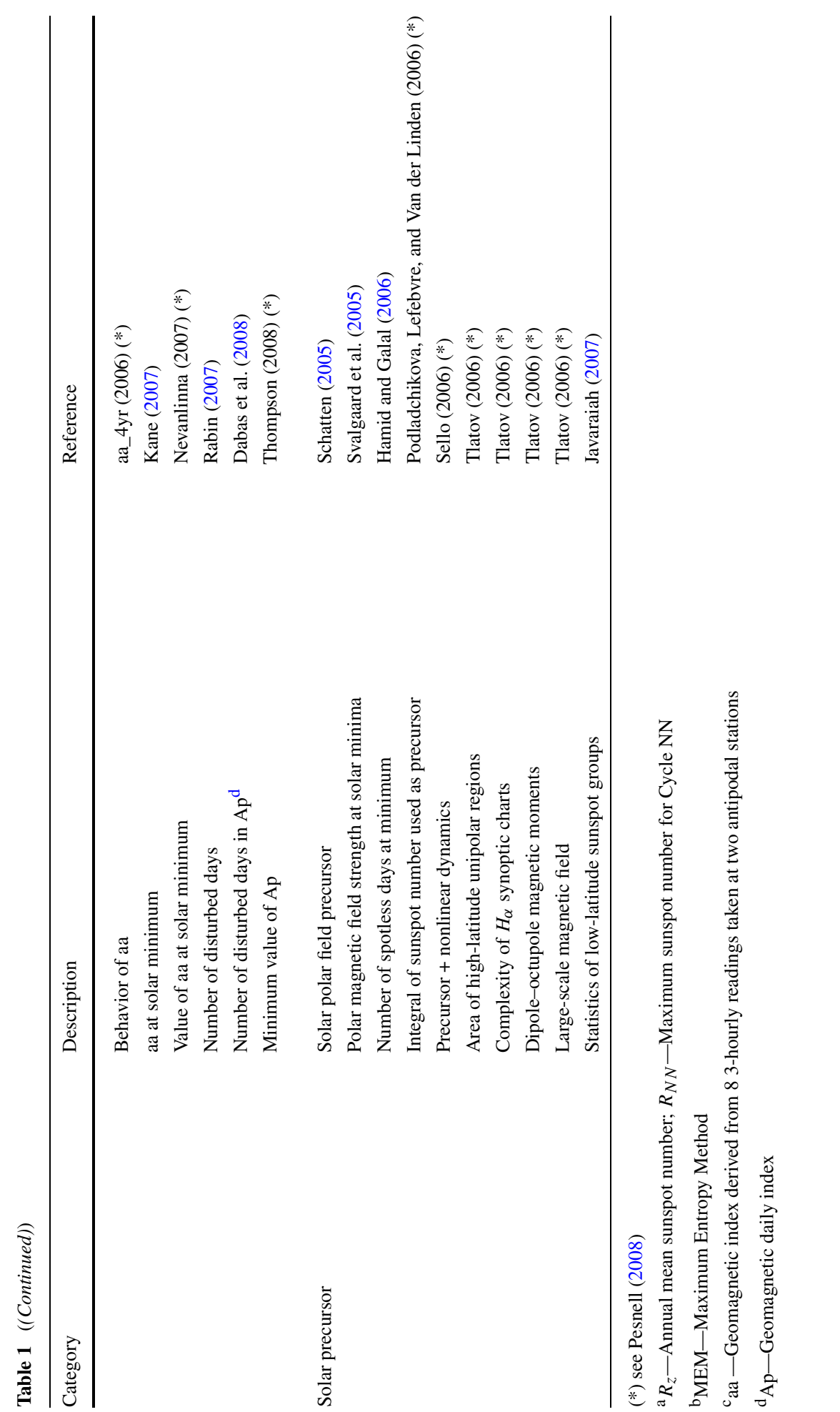


Howe et al. (2009) examined the evolution of the zonal flow pattern in the upper solar convection zone during the current extended solar minimum, and compared it with that during the previous minimum. Interestingly, the torsional oscillation progress seems to predict the solar cycle onset and it could have predicted the delayed onset of Cycle 24.

The panel is herewith split down the middle on the prediction of a bigger or a smaller than average solar cycle; thus the panel will re-evaluate conditions on the Sun every 3 months and update this prediction annually, or as things change.

A similar prediction panel was organized for Cycle 23. This time also predictions based on MHD dynamo models were presented. The first was Dikpati et al. (2006) prediction of a strong cycle. However it did not take long before another prediction was presented. Choudhuri et al. (2007) claimed their model suggests a weak Cycle 24. The models differ in the number of solar cycles they need to make Cycle 24: Choudhuri et al. (2007) suggested only one, but Dikpati et al. (2004) indicated several cycles.

The attempts to predict Cycle 24 have raised many fundamental questions about the solar dynamo, such as: - Why do dynamo models give different predictions? - Does the Sun has a memory and if so, for how long? - How far ahead is it possible to make predictions? - Is it at all possible to make predictions?

Bushby and Tobias (2007) state that it is not possible to make any predictions by using a mean-field dynamo, because of poor physical understanding of the dynamo, uncertainties in determining the transport coefficients, and, also, because of the non-linear chaotic nature of the solar dynamo.

In Cameron and Schussler (2007) the combination of the overlap of solar cycles and their amplitude-dependent rise time (Waldmeier's rule) introduces correlations in the sunspot number (or area) record, which account for the predictive skill of many precursor methods. This explanation requires no direct physical relation between the precursor quantity and the dynamo mechanism (in the sense of the Babcock-Leighton scheme or otherwise).

The difficulties in predicting also depend on the use of the sunspot number as the indicator of the solar cycle (Lundstedt 2009). The sunspot number is not a good indicator at low solar magnetic activity: during the so-called Maunder Minimum the sunspot number shows almost no activity, whereas the ${ }^{14} \mathrm{C}$ production rate shows solar modulation (Lundstedt et al. 2006). This was explained in Beer et al. (1998) by the fact that during the Maunder Minimum strong toroidal magnetic flux tubes (sunspots) were absent but weak ephemeral magnetic field (also indicated by the ${ }^{14} \mathrm{C}$ production rate) were present.

In his review on solar cycle forecasting, Hathaway (2009) emphasizes that both the predictability of the solar cycle and the ability of current dynamo models to provide predictions have been questioned, but he stresses that Cycle 24 will help to discriminate between some opposing dynamo models. The difficulties in predicting Cycle 24 clearly showed the lack of theoretical understanding and comprehensive data. Furthermore, Lundstedt (2009) points out the lack of precise definitions of solar magnetic activity, that it is envisaged to be achieved by using tools and concepts within Topology of Mathematics.

\section{Features and Models Relevant to Short-term Variations}

\subsection{Emergence of Magnetic Flux in the Solar Atmosphere and Active Region Formation}

During the first phases of active region (AR) formation, many physical processes are at work: convective collapse, magnetic coalescence, arch filament system (AFS) formation, plasma downflows along the rising flux tubes, decreasing upward velocities of the AFS 
arches, magnetic reconnection between the rising flux tubes and the ambient magnetic field lines, etc. (see, e.g. van Driel-Gesztelyi 2002 for a review).

In particular, observations indicate that active regions form due to the emergence of several small (radius $\sim 200 \mathrm{~km}$ ), intense $\left(500 \mathrm{G}, \sim 10^{18} \mathrm{Mx}\right.$ ) flux tubes, which are separate during the first phase of their appearance, but that soon cluster to $2-4 \times 10^{3}$ G over $100 \mathrm{~km}$ due to magnetic coalescence (Fragos et al. 2004 and references therein). The flux tubes clustering tendency takes place until new magnetic flux emerges from subphotospheric layers and vanishes as soon as their emergence ceases (Zwaan 1985).

However, despite all the observational evidence, it is still impossible to forecast if the emergence of flux tubes will cause the formation of a fully evolved and recurrent active region or will give rise to the formation of a structure which will disappear after a short time (i.e., hours, days). In this regard, high-resolution observations of emerging active regions have been recently carried out with the aim to distinguish, since the initial phases of magnetic flux emergence, whether the new forming region has been characterized by a short or a long lifetime. These studies have given new insights on the knowledge of physical phenomena occurring during this phase (see, e.g. Spadaro et al. 2004; Zuccarello et al. 2005) and might help to solve the above mentioned problem.

The characteristics shared by both the long-lived and short-lived active regions analysed are:

1. the first signatures of ARs emergence are initially observed in the outer atmospheric layers (transition region and corona) and later on (i.e. with a time delay of 6-7 h) in chromosphere;

2. the ARs appearance in the outer atmospheric layers seems to be simultaneous with the sudden increase of magnetic flux in photosphere;

3. the arches of the AFS are characterized by a decreasing upward motion during the AR's lifetime (see Fig. 7);

4. the downward plasma motion in the AFS loop legs is asymmetrical.

The differences observed between the long-lived and short-lived active regions are:

1. the appearance of the short-lived AR in photosphere and chromosphere is almost synchronous, while there is a time delay of $\sim 8$ hours between the appearance in chromosphere and photosphere for the long-lived AR;

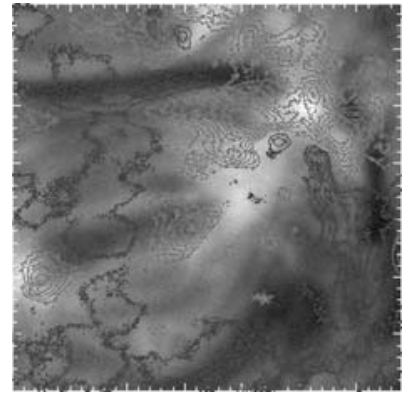

(a)

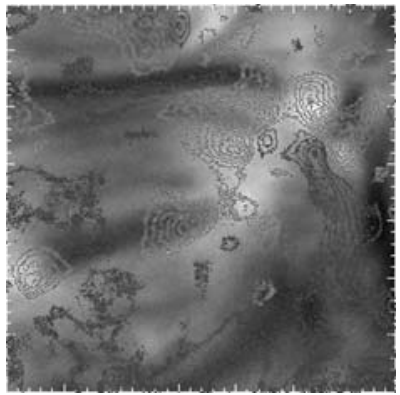

(b)

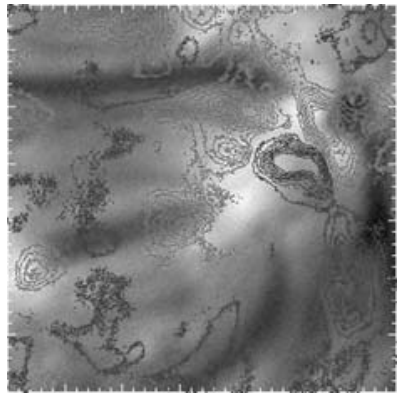

(c)

Fig. 7 Sequence of images, acquired by the THEMIS telescope along the profile of $H_{\alpha}$ line every ten minutes, showing the emergence of an arch filament system (AFS). Light grey contours indicate downflows, dark grey contours upflows 
2. during the AR formation the magnetic flux increases by about one order of magnitude in the long-lived AR and by only a factor 2 in the short-lived AR;

3. the displacement of the centre of symmetry of each polarity in the short-lived AR is mainly directed Westward, while it is diverging from the neutral line in the long-lived AR;

4. a higher plasma downflow is measured in the preceding leg in the short-lived AR, while it is observed in the following leg in the long-lived AR.

\subsubsection{Sunspots and Ephemeral Regions}

The most striking evidence of the solar activity is undoubtedly represented by sunspots. A very wide review about this phenomenon has been given by Solanki (2003). These dark magnetic features, visible in the solar photosphere, have a bipolar structure, usually with two main concentrations of magnetic flux of opposite polarity, aligned nearly in the East-West direction on the solar surface, in agreement with Joy's law.

Sunspots and sunspot groups are classified according to the observed photospheric features described by three parameters, proposed by McIntosh (1990): (1) Z-modified Zürich class, 7 items; (2) p-type of principal spot, 6 items; (3) c-compactness in the interior of the group, 4 items. According to the experimental evidences, only 60 sunspot group types are possible. The McIntosh classification proved effective in correlating the sunspot group characteristics with flaring probability. Furthermore, the relevant magnetic topology is described by the Mount Wilson classification (Hale and Nicholson 1938), characterized by eight classes based on magnetic polarity and magnetic complexity: $\alpha ; \beta ; \gamma ; \beta-\gamma ; \delta ; \beta-\delta$; $\beta-\gamma-\delta ; \gamma-\delta$.

Big and complex active regions are often formed by the coalescence of several emerging flux regions which emerge separately into the photosphere, but that are spatially and temporally close within a few days (Schrijver and Zwaan 2000). Harvey (1993) found an interval of 4-5 days between subsequent emergences of bipolar flux, while Harvey and Zwaan (1993) found an emergence rate 22 times higher within active regions than elsewhere. This trend of the regions of emerging flux to appear almost in the same locations involves the physics of the tachocline.

In emergence sites a wave-like structure has been observed, indicating that flux tubes emerge at several places, in a sea-serpent fashion, creating a mixed-polarity field where a substantial amount of flux may be cancelled (Bernasconi et al. 2002). A similar feature has been recently observed in sunspot penumbrae by Sainz-Dalda and Bellot-Rubio (2008).

The study of Lites et al. (1998) pointed out some fundamental properties of vector magnetic field in emerging flux regions: for instance, an emergence zone is found between the polarities of an emerging flux region. In such zone the field lines, directed from the positive polarity towards the negative one, present an East-West orientation with a slight inclination to the equator, in agreement with Joy's and Hale's laws, while the region evolves.

In the emergence zone, the magnetic field is more horizontal, i.e. it has an inclination $\gamma$ with respect to the photosphere ${ }^{1}$ which spans from $60^{\circ}$ to $120^{\circ}$, and it has weak field strength, between 200 and $600 \mathrm{G}$. Moreover, the emergence zone exhibits a strong upward motion, up to $1.5-2 \mathrm{~km} \mathrm{~s}^{-1}$. On the other hand, the magnetic field in footpoints is nearly vertical, with an inclination $\gamma<60^{\circ}$ or $\gamma>120^{\circ}$, and with intensity which can get 1500$2000 \mathrm{G}$ in pores and facular regions. Pores have a strong downflow of $1-2 \mathrm{~km} \mathrm{~s}^{-1}$, that

\footnotetext{
${ }^{1}$ Magnetic field coming out from just above the solar surface is assumed to have an inclination of $0^{\circ}$, while magnetic field directed towards the solar surface of $180^{\circ}$.
} 


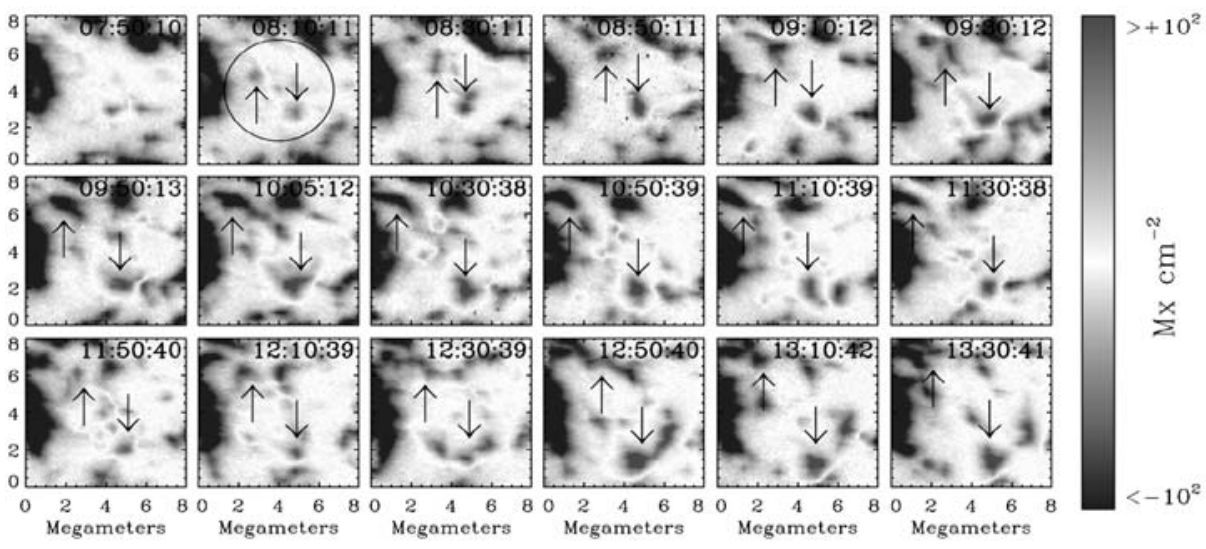

Fig. 8 Sequence of Na I D1 magnetograms, acquired by SOT aboard the Hinode satellite, showing the emergence of an ephemeral region. The separation of opposite polarity flux concentrations, as indicated by the arrows, grows in time

suggests that material brought to the surface by $\Omega$-loops falls down along the magnetic legs.

This information, together with other physical parameters, like temperature and filling factor, can be obtained by ad-hoc codes able to perform the synthesis and inversion of spectral lines formed in presence of magnetic fields (see, e.g., the SIR code in Table 2).

Indeed sunspots are not the only features associated with the photospheric magnetic field. Quiet network contains small-scale, short-lived bipolar magnetic regions with magnetic fluxes of $\sim 3 \times 10^{18} \mathrm{Mx}$ and typical size of $10 \mathrm{Mm}$, called "active ephemeral regions" due to their lifetime, i.e. the time they can be recognized as bipolar structures (Harvey 1993). Recent estimations show that the average lifetime of ephemeral regions spans from 8 to 16 hours (Hagenaar et al. 2003).

These bipolar regions emerge near the centre of supergranules, then their footpoints move fast apart and separate of $\sim 7000 \mathrm{~km}$ in about half an hour and later the speed of separation of the footpoints drops down to $\sim 0.4 \mathrm{~km} \mathrm{~s}^{-1}$ (see Fig. 8). Their decay appears complex, being strongly biased by the surrounding magnetic network.

Ephemeral regions are believed to have a common origin with active regions in the global dynamo (Harvey 1993), but it is also thought that they are generated locally everywhere by turbulent convection near the surface (Nordlund et al. 1992). A third way involves both the global dynamo and flux processing in the convective envelope. Thus, their origin remains rather speculative.

Recent high-resolution observations $\left(0.3^{\prime \prime}-1.2^{\prime \prime}\right.$ magnetograms $)$ have shown that the contribution of the unresolved small flux elements to the detectable flux into the network, due to flux coalescence, has an importance comparable to the total ephemeral regions rate for the total flux balance (Lamb 2009). These small-scale features also trace the evolution of the fields around supergranular network concentrations, showing the strong correlation between the formation and evolution of small-scale magnetic fields with surface flows and magnetic fields on the various scales. Moreover Parnell et al. (2009) have shown that all feature fluxes between $2 \times 10^{17}$ and $10^{23} \mathrm{Mx}$ follow a same power law distribution with slope $\sim-1.85$. This would imply that either all surface magnetic features are generated by the same scalefree mechanism, or that they are dominated by surface processes, suggesting a turbulent origin for the ephemeral regions. 


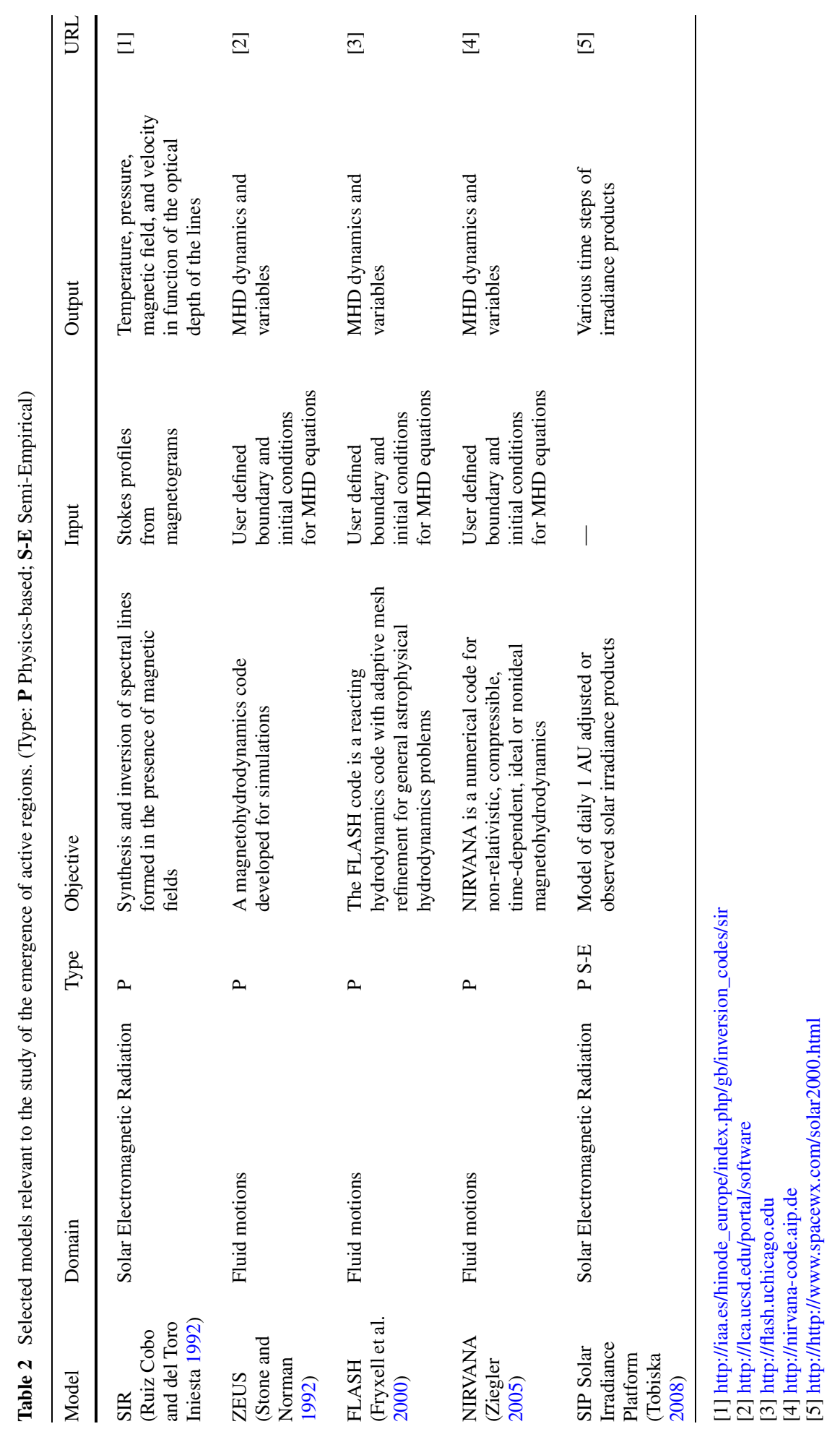




\subsubsection{Scientific Models for the Emergence of Magnetic Flux and Active Region Formation}

The current leading picture about the emergence of ARs on the solar surface is that they originate from strong toroidal magnetic fields generated at the tachocline, where they are stored until they traverse the entire convection zone via magnetic buoyancy and finally reach the photosphere. In order to understand some of the global features of ARs, usually buoyant flux tubes are considered embedded in a stratified layer and which then rise into the convective envelope.

Earlier calculations considered the dynamic evolution of buoyant toroidal flux tubes using a highly simplified "thin flux tube model" (Spruit 1981). In this model, the motion of the flux tube, treated as a 1D string developing into an emerging $\Omega$-loop, is determined essentially by buoyancy, magnetic tension, Coriolis force, and aerodynamic drag force, relying on the assumption that the tube radius is small compared to the local pressure height.

The results of such simulations indicated that, in order to obtain emerging tubes with properties consistent with the observed properties of ARs, the initial field strength has to be on the order of $100 \mathrm{kG}$, higher than the field strength in equipartition with convection, defined as $B_{\mathrm{eq}}^{2} / 2 \mu \approx \varrho v_{\text {conv }}^{2} / 2$, of the order of $\sim 10 \mathrm{kG}$. Moreover, these simulations showed that several asymmetries in the newly developing ARs, as the Joy law or the $p-f$ asymmetry, are due to the Coriolis force acting upon the emerging loops (Fan 2004).

More recently, full MHD simulations of 2D and 3D flux tubes have been carried out in order to test the underlying assumptions assumed in the thin flux tube model and investigate dynamical processes which cannot be addressed by such a simplified model. The presence of a minimum amount of magnetic twist seems to be essential for a flux tube to avoid the fragmentation of the tube during its rise (Moreno Insertis and Emonet 1996). Moreover, these simulations demonstrated clearly that flux tubes expand as they rise upward to the solar surface, so near the photosphere at least some of the assumptions of the thin flux tube model fail. In the framework of such kind of simulations, the concept of flux rope was introduced in order to remark the difference between a full 3D topological structure and the simple idealization of a 1D string (see Dorch 2002).

More complex MHD simulations involving convective motions and radiative transfer have been carried out by Cheung et al. (2007), who have found that the interaction between the magnetic flux tube and the external flow field has an important influence on the emergent morphology of the magnetic field. The emergence process of magnetic flux tubes with a flux content of $10^{19} \mathrm{Mx}$, comparable to a medium-sized ephemeral region, depending on the initial physical properties of the flux rope (e.g. field strength, twist, entropy, etc.), leads to results consistent with the complex patterns seen in high-resolution observations of the Hinode optical telescope.

Theoretical models approach the treatment of the ephemeral regions from two points of view: (1) the modelling of their emergence using MHD-simulation codes of emerging magnetic flux tubes (like FLASH, NIRVANA and ZEUS, whose main features are reported in Table 2), and (2) the statistical and simulated analysis of their emergence for what concerns the total balance of magnetic flux on solar surface (Simon et al. 2001).

\subsubsection{Prediction Models for the Emergence of Magnetic Flux and Active Region Formation}

Despite the fact that we know in deep details the various phases of the emergence of active regions, to date it is impossible to forecast their appearance on the solar surface. Yet, when they appear, we are not able to determine whether their duration on the solar atmosphere will be brief and featureless or they will develop into complex, flare-productive active regions: models able to forecast such behaviours simply do not exist. 
This situation could actually look disheartening, but recently some theoretical models have provided results which address indirectly but effectively this problem, related to space weather applications. In fact, the simplest approach is to predict the properties of the upcoming phases of the solar cycle or of the upcoming solar cycle via global dynamo models, by using old cycle data (see Sect. 3.3). In particular, what is inferred by these models is the average spot area, which strongly influences the total solar irradiance and is fundamental for space weather predictions (see, e.g., the SIP Solar Irradiance Platform in Tables 2 and 7).

Such investigations have been attempted by various methods: the most popular current method involves the use of polar fields from previous cycles as "precursors" of the next cycle (Svalgaard et al. 2005; Schatten 2005). In the light of their flux-transport dynamo model (Dikpati et al. 2004), that reproduces many solar cycle features, Dikpati et al. (2006) constructed a solar cycle strength prediction tool for making prediction of the amplitude of upcoming solar cycle 24. As stressed in Sect. 3.3, the analysis by Pesnell (2008) points out that the existing dynamo-based models do not have predictive capabilities yet and precursorbased ones result in significant discrepancies, leading to the need to consider solar and geomagnetic precursor as separate categories. Finally, whereas the prediction of solar activity climatology is to some extent successful, the prediction of exceptional activity events is still a challenge.

That overall approach to solar cycle prediction focuses on predicting changes in certain global characteristics of a cycle, without attempting to reproduce details that occur on smaller spatial scales and shorter time scales, in a way similar to the approach employed in global atmospheric dynamics. However, in all such considerations we have to keep in mind that all the relationships that may be used for prediction are valid only in a statistical sense. The split opinion of the NOAA/NASA Solar Cycle 24 prediction about whether the coming cycle would be high or low provides a good illustration about the "state of the art"-and may actually reflect intrinsic limitations of the method.

A basic step towards a sunspot predictive system is the automatic classification of sunspot groups from observations. Various attempts have been performed to automatically classify sunspot groups based on different approaches and all related to the prediction of the flaring probability, such as THEO (Shaw 1989), WOLF (Miller 1989) and the automated McIntoshbased classification system using SOHO/MDI Images (Colak and Qahwaji 2008) (see Table 5 in Sect. 4.2.2). Anyway, further advancements in the knowledge about the physics of sunspot formation and evolution are needed to provide the background for setting up a predictive system.

\subsection{Flares}

A solar flare is a sudden release of energy, from $10^{23} \mathrm{erg}$ in nanoflares to $10^{32} \mathrm{erg}$ in large two-ribbon flares, characterized by a time scale of few minutes in the impulsive phase and a duration of tens of minutes or few hours (see Fig. 9). During a flare, magnetic energy is converted into radiation across the entire electromagnetic spectrum, heating, particle acceleration and mass motions (see, e.g. Priest and Forbes 2000, 2002; Aschwanden 2004). Therefore solar flares (and often related coronal mass ejections) can be considered the most powerful explosions in the Solar System, and it is well known that radiation and particles emitted during these events may strongly interact with Earth magnetosphere and ionosphere.

Flares are classified according to the flaring area observed in $H_{\alpha}$ at the time of the maximum brightness, which determines the Importance class (5 items) and the Brightness qualifier ( 3 items). The Importance class is associated to the flare area expressed as millionths of the solar hemisphere: [0] (Subflare) $(10,100]$; [1] $(100,250]$; [2] $(250,600]$; [3] $(600$, 


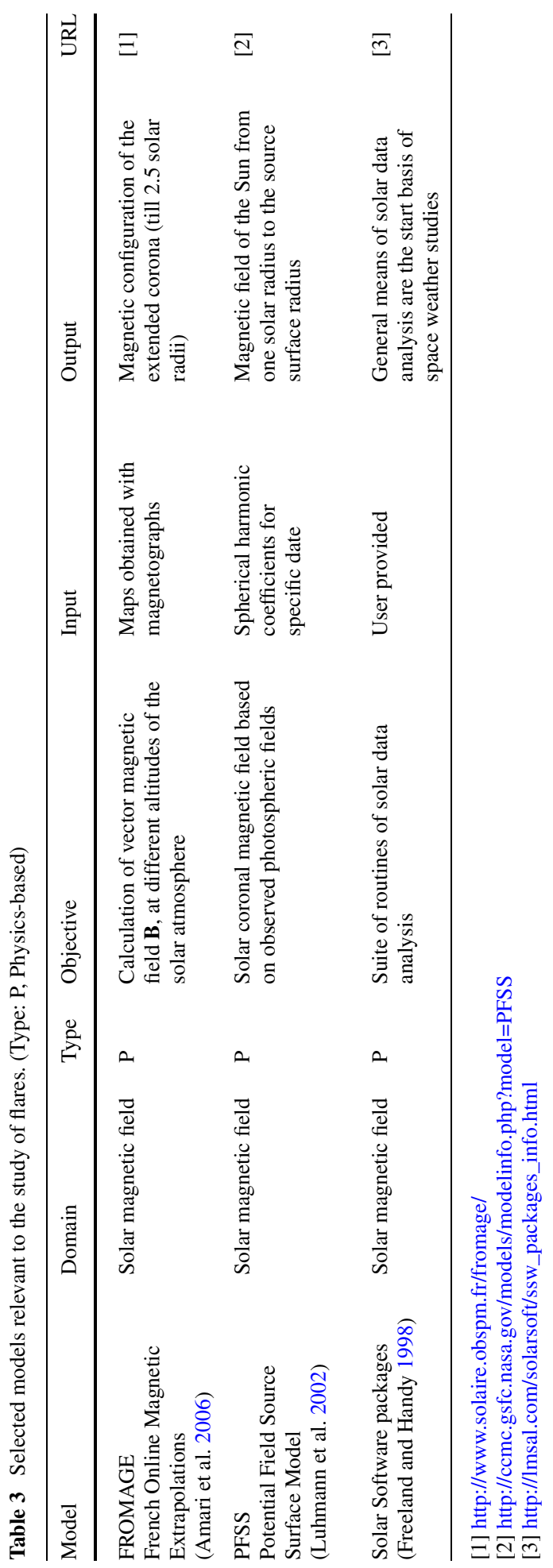




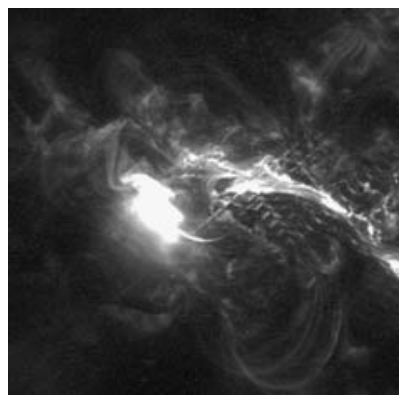

(a)

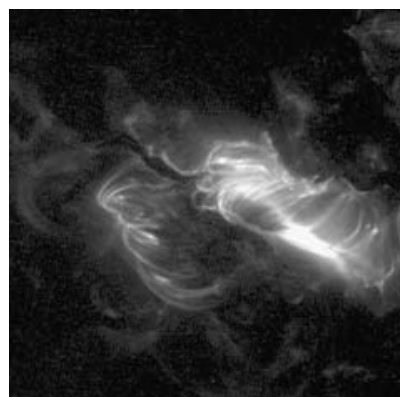

(b)

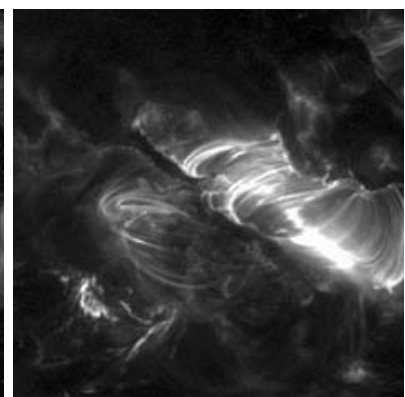

(c)

Fig. $919.5 \mathrm{~nm}$ TRACE images showing the evolution of an X17.2 flare occurred in NOAA 10486 on Oct 28, 2003. (a) 11:01 UT; (b) 11:48 UT; (c) 12:27 UT. The field of view of these images is $\sim 280 \times 280 \mathrm{Mm}^{2}$ $(\sim 384 \times 384 \operatorname{arcsec})$

1200]; [4] > 1200. The Brightness qualifier can be respectively: [F] Faint; [N] Normal; [B] Brilliant.

X-ray flares are classified according to the order of magnitude of the GOES X-ray (0.1$0.8 \mathrm{~nm})$ peak burst intensity $\mathrm{I}\left(\mathrm{W} \mathrm{m}^{-2}\right)$ measured at the Earth: [B] $I<10^{-6}$; [C] $10^{-6} \leq$ $I<10^{-5} ;[\mathrm{M}] 10^{-5} \leq I<10^{-4} ;[\mathrm{X}] I \geq 10^{-4}$.

We usually distinguish two typologies of solar flares:

Compact or simple-loop flares that generally occur in single loops whose shape and volume do not change significantly during the flare and that do not present particle emission.

Two-ribbon flares that occur in magnetic arcades and show at the lower atmospheric levels two areas of emission on both sides of the magnetic inversion line. The strands separate at $\sim 5-20 \mathrm{~km} \mathrm{~s}^{-1}$ while the filament lying between them rises higher and higher in the corona.

In order to fully understand the flare phenomenon, it is important to determine the magnetic configuration in the pre-flare phase (see, e.g., the FROMAGE and the PFSS codes in Table 3), by recognizing observational signatures such as for instance non-potential configurations in sheared magnetic fields and sheared structures in the corona (as sigmoids) or canceling magnetic features appearing just before or during the filament activation phase.

We still do not know how exactly energy is released and how the complex magnetic configuration becomes unstable and the flare is triggered. The main imputed mechanism is magnetic reconnection, which may be related to several phenomena, like emerging flux tubes, footpoint shearing motions, loop interactions and filament eruption.

The investigation of these phenomena implies the analysis of data acquired both from ground-based instruments and from satellites (see, e.g., the Solar Software Package, in Table 3 , for a suite of routines of solar data analysis).

It seems that the energy is released in the corona, but the site of the energy release, i.e. the current sheet, is expected to have dimensions $\left(\sim 10^{2}-10^{3} \mathrm{~m}\right)$ below the spatial resolution of current telescopes, therefore the identification of this structure is indirect, e.g. by means of the observations of cusps, in-falling dark blobs, inflows.

It is also unclear what happens after the energy is released. We do not know what fraction goes into heating, particle acceleration, and mass motions, and which effects are directly related to the energy release itself and/or with the subsequent transport effects.

As far as this last point is concerned, it is useful to describe the flare evolution as characterized by three steps: a) primary heating process; b) secondary heating process; c) upflow of chromospheric plasma (see Aschwanden 2004 for a more complete description). 
Primary heating process (particle acceleration and coronal plasma heating): it generally occurs in the corona above flare loop arcades, in the sites where magnetic reconnection occurs (e.g., null points, separator lines and separatrix surfaces) and magnetic energy is converted into plasma heating and particle acceleration.

In order to explain the observed flare dynamics, reconnection regimes should be unsteady and bursty, such as tearing mode and coalescence mode, and not steady state or quasi-steady, like the Sweet-Parker- or Petschek-type reconnection.

The secondary heating processes (beam-driven chromospheric heating) can be observed in $\gamma$-ray and hard X-ray (sometimes also in UV and white light) and take place when accelerated particles (electrons or protons) or thermal conduction fronts propagating downwards collide with the transition region and chromospheric plasma.

While the electron beam model, based on the thick target bremsstrahlung, considers fast electrons $(E \geq 20 \mathrm{keV})$ whose effective presence is witnessed by hard X-ray emission, in the case of proton beams, their role in the second heating processes cannot be ruled out by radiative signatures in hard X-rays. However, when these protons propagate towards the chromosphere, they may excite kinetic Alfvén waves that may produce the typical nonthermal velocities $(200-400 \mathrm{~km} / \mathrm{s})$ observed in flares.

In the thick-target model non-thermal electrons lose their energy in the transition region and upper chromosphere at heights of $\sim 2000-3000 \mathrm{~km}$. The primary electrons heat secondary electrons which dissipate their energy partially into collisional heating of the ambient ions, and in part into upward motions due to the overpressure. When the secondary electrons propagate upwards, an ambipolar field sets in, driving a return current of ions (due to the conservation of the charge neutrality).

The upflow of chromospheric plasma (chromospheric evaporation) starts when the overpressure causes an expansion characterized by thermal conduction fronts having a steep temperature gradient at the leading edges, probably propagating with an ion-sound speed. The chromospheric plasma is heated at a sufficiently rapid rate, so that it reaches coronal and flare temperatures $(\sim 3-35 \mathrm{MK})$ and expands upward into the coronal loops, which emit in soft X-rays. The heated plasma expands with a velocity of $v \sim 100-400 \mathrm{~km} / \mathrm{s}$ and can fill a loop of $L \sim 10-20 \mathrm{Mm}$ in $\sim 25 \mathrm{~s}$. Chromospheric evaporation occurs sequentially in overlying loops with increasing footpoint separation.

During the phase of chromospheric evaporation, observations show upflows of heated plasma (blueshift in Ca XIX) and simultaneously downflows of chromospheric plasma (redshift in $H_{\alpha}$ ), called chromospheric condensation, to balance the momentum.

The chromospheric response produces a gentle upflow for heating rates below $10^{10} \mathrm{erg}$ $\mathrm{cm}^{2} \mathrm{~s}^{-1}$ or an explosive upflow, with velocities greater than the sound speed, if the heating rate is greater.

Chromospheric evaporation can also be produced by heat conduction fronts, which is important for flares without detectable hard X-ray emission.

Once the flare passes its peak in soft X-ray emission, plasma cooling processes start to dominate over heating. When the plasma cools down from the initial 10-30 MK temperatures of the peak of the flare down to 1-3 MK, the postflare loop system becomes detectable in EUV.

\subsubsection{Scientific Models for Solar Flares}

Theoretical models of solar flares are based on the evidence that they are triggered by an instability of the magnetic field which evolves into a more stable state by changing and reconnecting its topology (see Table 4). 


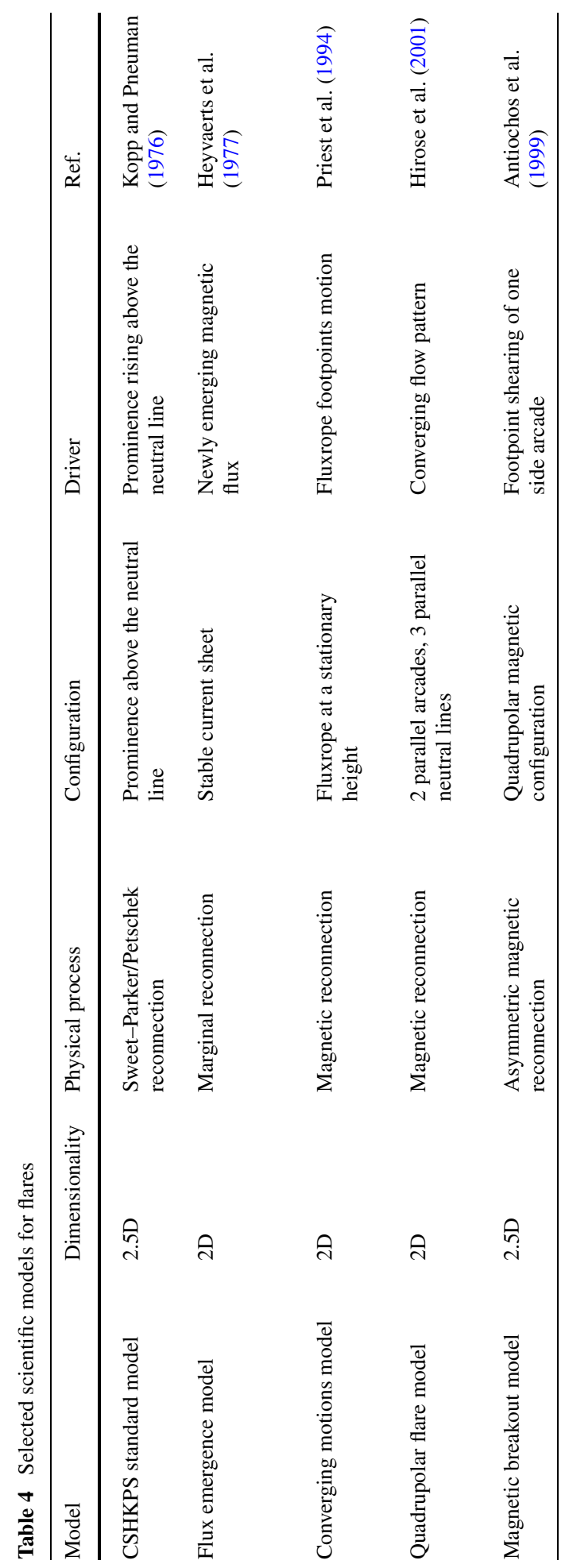


The standard model, also called CSHKP 2D magnetic reconnection model (Carmichael 1964; Sturrock 1966; Hirayama 1974; Kopp and Pneuman 1976) assumes that the initial driver is a rising prominence above the neutral line. The rising filament stretches a current sheet, where the Sweet-Parker or Petschek reconnection can occur, and carries an electric current parallel to the neutral line, inducing a magnetic collapse, accompanied by lateral inflow of plasma, on both sides of the current sheet. In the reconnection region the magnetic energy dissipation takes place, causing heating of the local coronal plasma and acceleration of nonthermal particles. These processes produce thermal conduction fronts and nonthermal particles that heat the chromospheric footpoints of the newly reconnected field lines. Chromospheric plasma evaporates and fills the newly reconnected field lines with overdense heated plasma, which becomes soft X-ray emitting with temperatures of $T_{e} \sim 10-40 \mathrm{MK}$ and densities of $n_{e} \sim 10^{10}-10^{12} \mathrm{~cm}^{-3}$.

This 2D model describes the evolution in a vertical plane, while the evolution along the third dimension (i.e. along the neutral line) can be repeated for multiple flare loops. The extension in the third dimension is not continuous but rather highly fragmented into temporary magnetic islands (tearing-mode instability). This model however does not specify how the initial magnetic configuration becomes unstable and is not appropriate for quadrupolar flare loop interactions and 3D nullpoint topologies.

Flux emergence is the driver in the model of Heyvaerts et al. (1977), where we can distinguish three phases: (1) new magnetic flux emerges and a current sheet forms between the old and the new flux; (2) the current sheet loses equilibrium at a critical height and turbulent resistivity causes particles acceleration and triggers chromospheric evaporation; (3) the current sheet reaches a new steady state with marginal reconnection. A requirement of this model is the pre-existence of a stable current sheet for a day or more. However, numerical simulations indicate that the current sheet reconnects almost as quickly as it is formed.

In the model based on converging motions (Priest et al. 1994) there is initially a flux rope at a stationary height, and when the two footpoints of the field lines that envelope the flux-rope approach each other, the system evolves through a series of force-free field configurations. When the footpoint distance passes a critical point, the flux rope jumps in height, forming a current sheet below it. This analytical model is $2 \mathrm{D}$ and is based on converging flows far from being realistic, because typical photospheric flows are of $\sim 1 \mathrm{~km} / \mathrm{s}$, much slower than those assumed in the model, or may be randomly oriented.

In the 2D quadrupolar flare model (Hirose et al. 2001) the initial configuration consists of two parallel arcades and three parallel neutral lines. The driver is a converging flow pattern that pushes the two arcades together. The X-point above the middle neutral line supports a filament. When the two arcades are pushed together, the filament transforms into a thin vertical current sheet, which then becomes unstable due to tearing-mode instability, triggering anomalous resistivity and fast reconnection.

The magnetic breakout model (Antiochos et al. 1999) is based on the same initial quadrupolar magnetic configuration, but the evolution is asymmetric due to reconnection occurring on one side. The asymmetric evolution is due to footpoint shearing of one side of an arcade, and reconnection between the sheared arcade and the neighboring (unsheared) flux systems triggers the eruption.

In conclusion, in flare models there are essentially two locations of drivers: (1) above the flare site (rising filament); (2) below the flare site (photospheric emergence, convergence flows, shear flows). The three photospheric drivers can be discriminated by their directions: (1) flux emergence corresponds to a flow in the vertical direction $\left(v_{z}\right)$; (2) convergence flows are counter-directed perpendicular to the neutral line $\left(+v_{x},-v_{x}\right)$; (3) shear flows are counter-directed parallel to the neutral line $\left(+v_{y},-v_{y}\right)$ (Aschwanden 2004). 


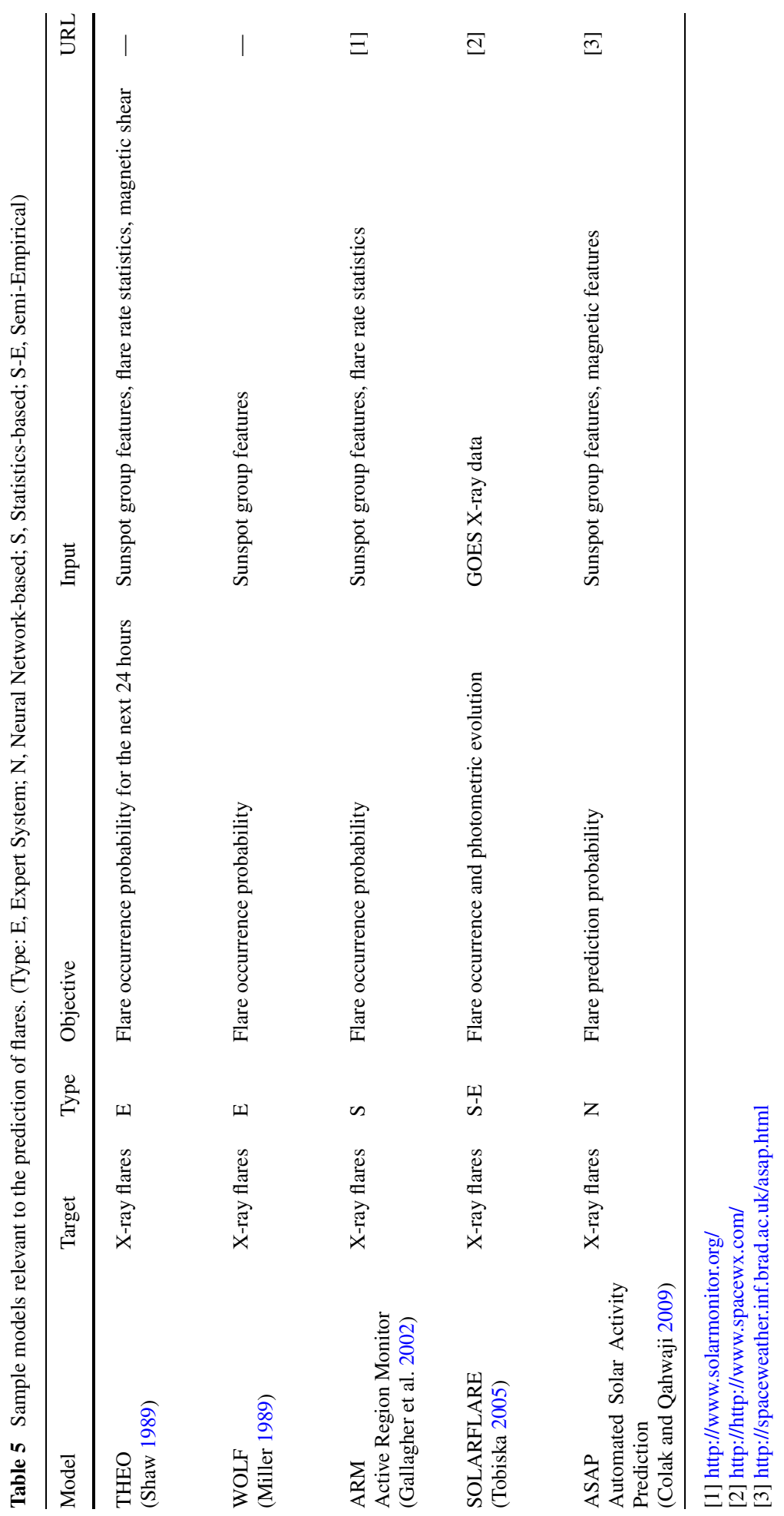




\subsubsection{Prediction Models for Solar Flares}

As already stated in this section, there are still many unsolved questions concerning our understanding of solar flares. Besides these, an important problem concerns the ability to forecast flare occurrence.

In this regard, we would like to stress that the capacity of predicting the occurrence of solar flares is important from two different points of view. The first is related to the fact that, if we want to perform high-resolution observations of all the phases of a flare, starting from the pre-flare phase, through the impulsive, to the main phase, it is necessary to know the exact location on the solar disk where instruments should be pointed. The second regards the effects that some flares, especially those related to coronal mass ejections, might have on the Earth environment, on radio communication systems, GPS positioning systems, electrical distribution networks, as well as on satellites orbiting around the Earth.

The efforts made until now to forecast solar flares are often based on different conceptual procedures (see, e.g., Shaw 1989; Miller 1989; Moon et al. 2001; Wheatland 2001; Gallagher et al. 2002; Jensen et al. 2004; Tobiska 2005; Wheatland 2005; Jing et al. 2006; Ternullo et al. 2006; Barnes et al. 2007; Falconer et al. 2007; Georgoulis and Rust 2007; Qahwaji and Colak 2007; Contarino et al. 2009; Colak and Qahwaji 2009).

A first attempt led to the development of THEO(PHRASTUS) (Shaw 1989), an expert system aimed at predicting X-ray flares for the next 24 hours based on the McIntosh sunpost classification and additional features of sunspot groups not described by the classification, such as historical flare rates, spot growth and spot activity, i.e. rotation and magnetic shear. A simpler expert system for sunspot classification and solar flare prediction (WOLF) was set up by Miller (1989).

Wheatland (2001) made a statistical analysis of the numbers of flares produced by individual active regions and of their flaring rates, showing that the distributions are approximately exponential, with an excess number of active regions with low flare numbers and low flaring rates. A large number of active regions exhibits variation in flaring rate during the transit on the solar disk. A good model for the observed waiting-time (i.e., time between events) distributions is provided by a constant Poisson process (see also Moon et al. 2001).

Using high-resolution observations at the Big Bear Solar Observatory Active Region Monitor (http://www.solarmonitor.org/), Gallagher et al. (2002) obtained magnetic gradient maps derived from GONG longitudinal magnetograms, as a useful diagnostic of flare activity.

Jensen et al. (2004) proposed the application of local-area helioseismic methods for the prediction of space weather. Specifically, nowcast information on solar sub-surface flows (curl and divergence of the flow field) derived from local-area helioseismic analyses applied to SOHO and GONG data is fed to an artificial neural network for flare prediction.

Based on the GOES X-ray data, the SOLARFLARE model set up by Tobiska and Bouwer (2005) predicts the evolution of the flare irradiance by providing flare rise, timing and magnitude of the peak fluxes, decay to half maximum, termination at background levels and a quantitative flare categorization.

Wheatland (2005) applied a Bayesian approach to solar flare prediction that uses only the event statistics of flares already observed. The skill score, i.e. the improvement of the forecasts over a constant forecast given by the average observed rate, obtained when predicting the daily GOES events in the period 1976-2003 was respectively 0.272 for M-X events and 0.066 for $\mathrm{X}$ events (1.0 represents the perfect forecasting).

Jing et al. (2006) using line-of-sight Michelson Doppler Imager (MDI) magnetograms put into correlation three parameters of magnetic fields (the mean value of spatial magnetic 
gradients at strong-gradient magnetic neutral lines; the length of strong-gradient magnetic neutral lines, and the total magnetic energy dissipated in a layer of $1 \mathrm{~m}$ during $1 \mathrm{~s}$ over the active region's area) and the flare productivity of solar active regions. The positive correlations indicated by their result confirm the dependence of flare productivity on the degree of non-potentiality of active regions. In this regard, Falconer et al. (2007) proposed a measure of total non-potentiality in multipolar active regions of any degree of magnetic complexity, from the vertical field component of vector magnetograms or also from the line-of-sight component.

Ternullo et al. (2006) carried out a statistical analysis on sunspot groups hosting M and $\mathrm{X}$ flares and deduced that the conditions which characterize active regions hosting $\mathrm{M}$ and $\mathrm{X}$ flares are: i) Zürich class D, E, F; ii) penumbra in the largest sunspot characterized by a large asymmetry and a diameter greater than 2.5 degrees; iii) $\beta, \beta \gamma$ and $\beta \gamma \delta$ magnetic configuration.

A totally different approach was followed by Georgoulis and Rust (2007), who measured the effective connected magnetic field $B_{\text {eff }}$, related to the magnetic connectivity between flux concentrations in MDI magnetograms. This has been found as an efficient flare-forecasting criterion: in fact, the flaring probability exceeds 0.95 for M-class and X-class flares if $B_{\text {eff }}>$ $1600 \mathrm{G}$ and $B_{\text {eff }}>2100 \mathrm{G}$, respectively.

Barnes et al. (2007) applied the statistical approach of discriminant analysis to solar flare forecasting, to provide the probability that e.g. a set of variables relevant to the photospheric magnetic field, derived from vector magnetic measurements, belong either to the group of solar active regions producing flares within 24 hours or to non-flaring ones. The skill score obtained for tests was 0.252 for the M-flare threshold, comparable to that obtained for the Wheatland (2005) and for the Space Weather Prediction Center (http://www.swpc.noaa.gov/) methods for M-X flares, and 0.123 for X-flares.

An automatic short-term solar flare prediction system based on machine learning and sunspot associations has been set up by Qahwaji and Colak (2007). The system can provide a prediction up to six hours in advance by analysing the latest sunspot data, and it confirmed the direct relation between the flare production and certain McIntosh classes like Ekc, Fki and Fkc.

Contarino et al. (2009), taking into account the results obtained by Ternullo et al. (2006) and the parameters that might be deduced in real time by photospheric observations performed at INAF-Catania Astrophysical Observatory, carried out a flare-forecasting Campaign, using an algorithm based on the Poisson statistics. The results obtained during the period 2004-2007 indicate that there is in general a satisfactory correlation between the event probability inferred by the flare-forecasting procedure and the real flare occurrence.

The most recent development is ASAP (Automated Solar Activity Prediction), a hybrid computer platform using machine learning and solar imaging for automated prediction of significant solar flares (Colak and Qahwaji 2009; http://spaceweather.inf.brad.ac.uk/ asap.html). ASAP is an evolution of the prediction system by Qahwaji and Colak (2007). SOHO/MDI Continuum and Magnetogram images are used to detect and classify sunspots. This information is fed to a hybrid system composed of two neural networks, which provide both the flaring probability of each sunspot group and the relevant flare intensity probability. The success rate for the final flare prediction is around $70 \%$.

\subsection{Coronal Holes}

There are two classes of coronal hole models of interest to the scientific community: (1) near short-term and (2) climatological models. 
The first kind of models aims to understand the intrinsic nature of holes.

The second kind, which should provide an extended view of what to expect over time in terms of coronal hole characteristic parameters (percentage of corona covered, hole categories, latitudinal/longitudinal distribution, time evolution, periodicities, relationship with other solar activity phenomena, macro and micro-effects in the solar system, etc.) are still not completely ready (Bohlin and Sheeley 1978; Navarro-Peralta and Sanchez-Ibarra 1994; Wang et al. 1996; Bravo et al. 1998b; Ikhsanov and Ivanov 1999; Obridko and Shelting 1999; Luhmann et al. 2002).

Nevertheless, such a kind of work started with the discovery of low-density areas in the solar corona (Waldmeier 1981, and references therein) and continued after the Skylab coronal hole identification (see Zirker 1977, for a monograph from the Skylab Solar Workshop I) by using Fe XIV (530.3 nm) coronal emission, He I (1083 nm) absorption line, K-coronagraph, X-ray and EUV observations.

Nowadays, three broad classes of coronal holes are identified: (1) polar (lifetime: a significant fraction of the heliomagnetic semi-cycle), (2) isolated (one Carrington Rotation or more), and (3) transient (few hours to few days) holes.

Data mainly derived from He I observations by the vacuum solar telescope at the Kitt Peak Observatory (U.S.A.), implemented with magnetograms and $\mathrm{H}_{\alpha}$ synoptic charts, were used to derive a catalogue of coronal hole occurrence (Sanchez-Ibarra and Barraza-Paredes 1992) from 1970.1 to 1995.4 (available at the National Geophysical Data Center - Boulder - U.S.A.). For practical purposes the coronal holes, identified on Carrington Rotation (CR) maps, were divided into two categories: (1) Extended Polar Coronal Holes (EP-CHs), if a part of the polar coronal hole is seen below $\pm 60^{\circ}$ heliographic latitude, and (2) Isolated Coronal Holes (I-CHs), for those not connected with any polar $\mathrm{CH}$ and occurring at latitudes below $\pm 60^{\circ}$ (see Fig. 10). Recently, Bilenko (2002, 2004) extended the work to 2002 (but the catalogue is not yet available). The extensive use of the catalogues, together with the study of individual holes, led to relevant information for the development of climatological models in the next future, which should be tested with "in situ" observations. Shortly, they can be summarized as follows.

\subsubsection{Polar Coronal Holes}

Present knowledge on polar $\mathrm{CH}$ occurrence/evolution demonstrates that their life is closely connected with the solar polar field reversals (Fox et al. 1998). More precisely, they expand towards the heliographic equator during the declining phase of each Schwabe cycle (which has an average length of $\sim 11$ years, but with considerable variation from one cycle to the next) and shrink back to the poles in the rising one of the successive cycle (Das et al. 1993). Polar CHs disappear during the maximum solar activity phase for a certain time and reappear with an inverted magnetic field polarity after it. Harvey and Recely (2002) gave a detailed description of the polar hole evolution from the maximum phase of cycle 22 to the one of cycle 23. Figure 10 illustrates the Northern polar coronal hole formation during 1991, where the evolution of a high-latitude I-CH $\left(\sim 60^{\circ} \mathrm{N}\right.$; the so-called pre-polar hole) and its subsequent expansion into the polar region can be seen. Its spatial dynamics (see also Bilenko 2002) supports the hypothesis that polar holes develop from the formation and growth of I-CHs located at middle/high latitudes (see, for instance, Webb et al. 1984; Fox et al. 1998) and tend to decrease with the emergence of the first larger active regions belonging to the new solar cycle. Nevertheless, only direct "in situ" observations can definitively clarify the topic. From the study of Harvey and Recely (2002), it is clear that the hemispheric time history of polar holes is not the same. Moreover, the area of the two holes differs and the magnetic flux density is higher in the smaller one. However, 
Fig. 10 Boundaries of the coronal holes observed during the indicated CRs. The forming Northern polar coronal hole (positive polarity) is shown by the heavy curve. The Northern polar fields reversed in January-March 1991. Right: the polar projection of the pre-polar coronal hole and its expansion to include the pole by CR 1843 . Left: coronal hole boundaries shown in the Carrington longitude-sine latitude coordinate system. I-CHs are easily seen there together with the EP-CH in CR 1843 (adapted from Harvey and Recely 2002)
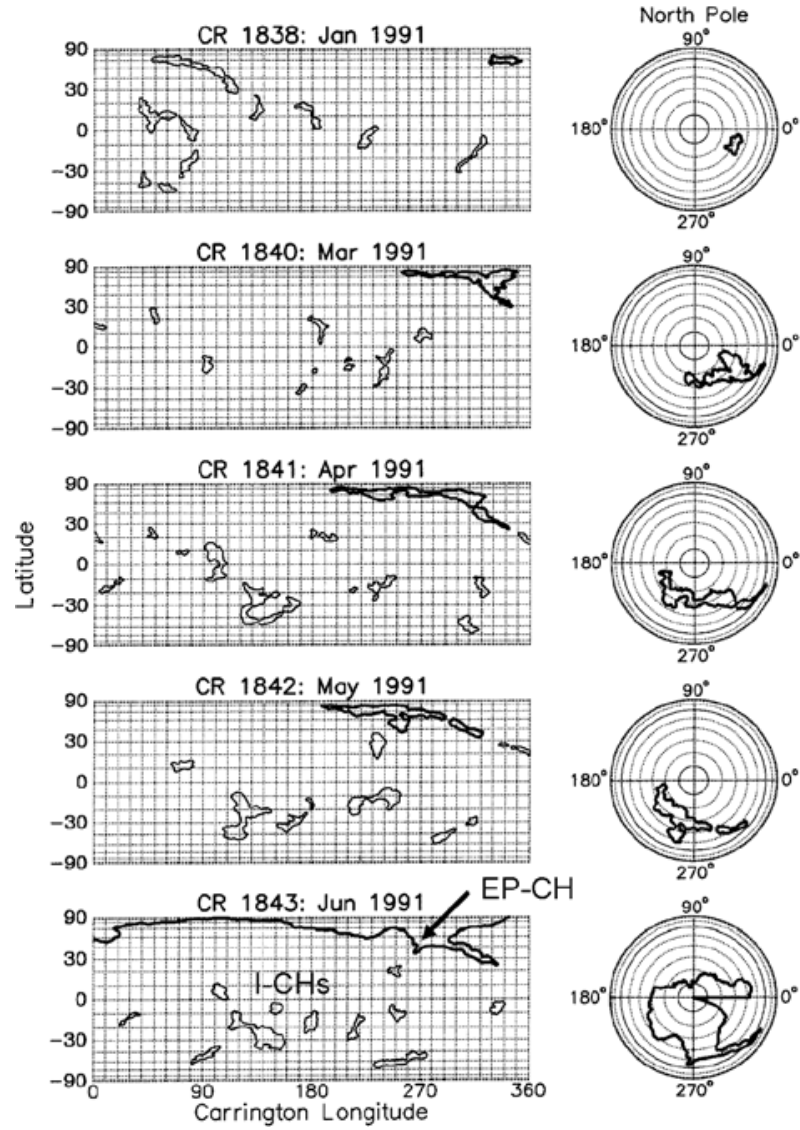

- the magnetic flux amplitude and long-term variability is practically the same, except during the initial stage;

- the fraction of absolute net magnetic flux relative to the total one peaks during the activity minimum.

Moreover, the longitudinal positions of equatorial extensions of polar holes (also called polar lobes) avoid the ones related to sunspot groups and active regions (see Sanchez-Ibarra 1990, for an early work).

\subsubsection{Isolated Coronal Holes}

The time history of non-polar coronal holes is very different from the polar hole one. The holes isolated from the poles show an occurrence rate that tends to follow the Schwabe cycle (Insley et al. 1995; Luo et al. 1997; Hofer and Storini 2003) and they are generally located at the so-called active region latitudes. The majority, but not all, of the ICHs follow the hemispheric polarity rule (they match the polarity of the hemispheric polar field, Bohlin and Sheeley 1978; Harvey and Sheeley 1979; Hofer and Storini 2002; Bilenko 2002). Moreover, some I-CHs show a close connection with active regions during their time evolution (McIntosh 1992; McIntosh et al. 1992; Wang et al. 1996; Bravo et al. 1998a; Storini and Hofer 1999; Hofer and Storini 2003). 
There are three relevant findings that should be understood in detail for the comprehension of the global solar activity cycle and the development of climatological models:

1. the North/South asymmetry in the hemispheric presence of long-lived I-CHs ( $>5$ Carrington Rotations) found during cycles 21 and 22, with a Northern hemisphere dominance (Hofer and Storini 2002);

2. the formation and disappearance of CH cluster structures in longitude (Bilenko 2004);

3 . the relevance of the recently identified two classes of long-lived I-CHs during the oddeven couple of Schwabe cycles 21 and 22 (Storini and Hofer 2003a, 2003b). Class I, occurred around the maximum phase of solar activity, is made up by long-lived I-CHs of both magnetic field polarities, lying on a medium-term (time extent: 2-4 years) diagonal area, from middle heliographic latitudes towards the equator and beyond. Class II is characterized by long-lived I-CHs having a high probability to occur on a long-term (1618 years, i.e. lasting for more than a solar activity cycle) diagonal region, one for each magnetic field polarity.

Bilenko (2004) suggested that "CHs connected with active regions reflect active region time-latitude and time-longitude distributions on the solar disk" while the others follow "the magnetic field dynamics". Storini et al. (2006) demonstrated that indeed long-lived I$\mathrm{CHs}$ of Class I are related to active regions of energetic X-flares during the maximum phase of solar activity, while those of Class II act as boundaries of energetic X-ray flare regions in the Northern hemisphere during the odd cycle and in the Southern one during the even cycle.

\subsubsection{Transient Coronal Holes}

Rust (1983) analyzing soft X-rays from SKYLAB measurements identified transient dimmings in the X-ray flux which he called "transient coronal holes". YOHKOH and SOHO images suggest that these regions with depleted emission in the X-rays and at EUV wavelengths are closely related to the aftermath of eruptive events such as flares, filaments and CMEs (e.g. Harvey 1996; Hudson and Webb 1997; Thompson et al. 1998; Zarro et al. 1999; Sterling et al. 2000; Harra and Sterling 2001, among others). The most common interpretations of these dimmings are coronal shock waves, evacuations of coronal matter due to the lift-off of prominences/CMEs becoming visible as expanded "virtually open field" regions and/or magnetic reconfigurations involving magnetoconvection-driven magnetic reconnection, reconnection fronts or MHD-solitons (Wang et al. 2002; Webb et al. 2000; McIntosh et al. 2007; Attrill et al. 2007; Wills-Davey et al. 2007). Simultaneous observations of EUV waves detected by the twin STEREO satellites at high-cadence (2.5 minutes) $171 \AA$ images showed significant differences in the wave appearance for the two viewpoints that were interpreted as a fast-mode shock wave triggered by expanding loops associated with the CME lift-off (Patsourakos et al. 2009).

\subsubsection{Relevance to Solar Climate Models}

All the aforementioned $\mathrm{CH}$ features have a strong influence on the time variability of the solar system properties. This implies that some findings of the solar/interplanetary physics, which are still misunderstood, can be clarified with future space research programs. For example, from long ago differences were claimed in the solar system conditions during even- and odd-numbered Schwabe cycles, but a detailed explanation for them was not still given. This certainly makes the development of climatological models difficult for the global 
solar activity (Maravilla et al. 2001) and for their use in space weather and climate forecasts. Nevertheless, the analysis of the interplay between $\mathrm{CHs}$ and sunspot/active regions/filament eruptions/CMEs will help to find a good model for the even-odd cycle couple. Such a model is also of interest for issues related to the cosmic ray physics in the heliosphere.

\subsection{The Solar Wind}

\subsubsection{Historical Background}

A solar influence on the Earth upper atmosphere was suggested by Lord Carrington in the nineteen century, but for clear evidence of a continuous outflow of fast particles from the Sun, we must go to the fifties of last centuries, when Biermann, studying the shape of the cometary ion tails, deduced a particle flow from the Sun, with an average speed of around $475 \mathrm{~km} / \mathrm{sec}$ (Biermann 1951, 1953).

At that time the coronal temperature was already known to be in the $1-2 \times 10^{6} \mathrm{~K}$ range, a temperature high enough to let the coronal gas to escape from the Sun.

In 1957 Chapman calculated the density of a static conductive corona with a temperature of $10^{6} \mathrm{~K}$ at the solar surface, obtaining a large density and pressure at infinity, contrarily to the observations.

Parker (1958) used the simplifying assumptions of steady flows and a constant temperature; the equations of motion in the spherically symmetric case, for an electron-proton gas, may be written in the form

$$
\begin{aligned}
\rho v \frac{d v}{d r} & =-\frac{d p}{d r}-\frac{G M_{\odot} \rho}{R_{\odot} r^{2}} \\
\frac{d}{d r}\left(\rho v r^{2}\right) & =0, \quad p=2 \kappa T \rho / m_{p},
\end{aligned}
$$

where $v$ is the velocity, $\rho$ the density, $p$ the pressure, $\kappa$ Boltzmann constant, $T$ the temperature, $m_{p}$ the proton mass, $M_{\odot}$ the solar mass, $R_{\odot}$ the solar radius and $G$ Newton's constant. The radial distance, denoted with $r$, is normalized to the solar radius.

For a static atmosphere, the pressure profile implies, as said above, a non-vanishing value for the pressure at large distances $p_{\infty}=p_{0} e^{-G M_{\odot} m_{p} / 2 R_{\odot} \kappa T}$.

Parker showed that these equations have solutions, $v(r)$, which are subsonic (i.e., from $r=1$ to $r=\infty)$, solutions which are supersonic and two solutions which pass trough the sonic point $\left(v=\sqrt{2 \kappa T / m_{p}}, r=G M_{\odot} m_{p} / 4 \kappa R_{\odot} T\right)$, one decreasing, one increasing. Among these solutions only the latter has vanishing density and pressure at large distances: this is, according to Parker, the correct solution for the solar case.

The value of $r$ at the sonic point, the critical radius, for $T=1.0 \times 10^{6} \mathrm{~K}$, is $r_{c}=5.8 R_{\odot}$. Hence the expanding corona, in Parker's model, becomes supersonic at a heliocentric distance of only a few solar radii.

The existence of a supersonic outflow, with a flux density of a few times $10^{8} \mathrm{~cm}^{-2} \mathrm{~s}^{-1}$, was confirmed by the measurements of the Luna 2 and Explorer (1961) spacecraft (Gringauz et al. 1960, 1961; Bonetti et al. 1963).

The outflowing coronal plasma has a profound influence on the magnetic field. Because of the high electrical conductivity of the plasma, the magnetic field is frozen in the expanding corona and is therefore carried by it in the interplanetary space. Being the field rooted in the solar surface, the combination of the radial expansion with the rotation of the Sun gives a spiral shape to the field lines; this was calculated by Parker (1958) assuming a steady and spherically symmetric expansion, in an interval of $r$ where $v$ could be taken as approximately constant (Archimedean spiral). 


\subsubsection{The Heliospheric Structure}

A general picture of the heliosphere can be obtained considering that this region is simply an extension of the solar corona. Not static, of course, because the Sun, where the magnetic field is rooted, is rotating, and because the plasma, owing to its temperature, is outflowing.

In fact the observations have shown that the magnetically open regions (coronal holes; see Sect. 4.3) give rise to fast solar wind (Noci 1973; Krieger et al. 1973; Bell and Noci 1973; Neupert and Pizzo 1974). On the contrary, the magnetically closed regions (streamers) end in a current sheet. At solar minimum the resulting simple configuration is depicted schematically in Fig. 11. The interplanetary magnetic field (not shown in the figure) has a spiral shape, because of the solar rotation, as explained in Sect. 4.4.1.

The maximum activity phase is characterized by a decrease in the extent of the polar holes, while a wide interval of latitudes around the equator is covered by streamers. In this latitude interval only small coronal holes existing among streamers (often the extension of a polar hole) give rise to fast solar wind, which therefore is present, in the interplanetary space, only at some solar longitudes.

This picture of the heliospheric structure has been confirmed beautifully by the Ulysses spacecraft (Fig. 12).

In conclusion, the solar corona, at activity minimum, is characterized by the streamer belt, which, extending out into the interplanetary space around a current sheet, divides the heliosphere in two opposite polarity regions. Fast wind $(\sim 780 \mathrm{~km} / \mathrm{s})$ flows in both unipolar regions on the sides of the current sheet, slow wind ( $\sim 300-400 \mathrm{~km} / \mathrm{s})$ being confined to the borders of those regions, so that a measuring spacecraft observes slow solar wind only when it is close to the current sheet (Fig. 11). Similarly the Earth is embedded for some time in the slow wind flowing along the current sheet, but it is swept, at each solar rotation, by streams of fast wind originating from the low-latitude extensions of the polar holes. The interaction of the low-latitude fast streams with the Earth magnetosphere causes a recurrent magnetic disturbance. (The cause of this phenomenon had been a mystery until the discovery of the sources of the fast wind.)

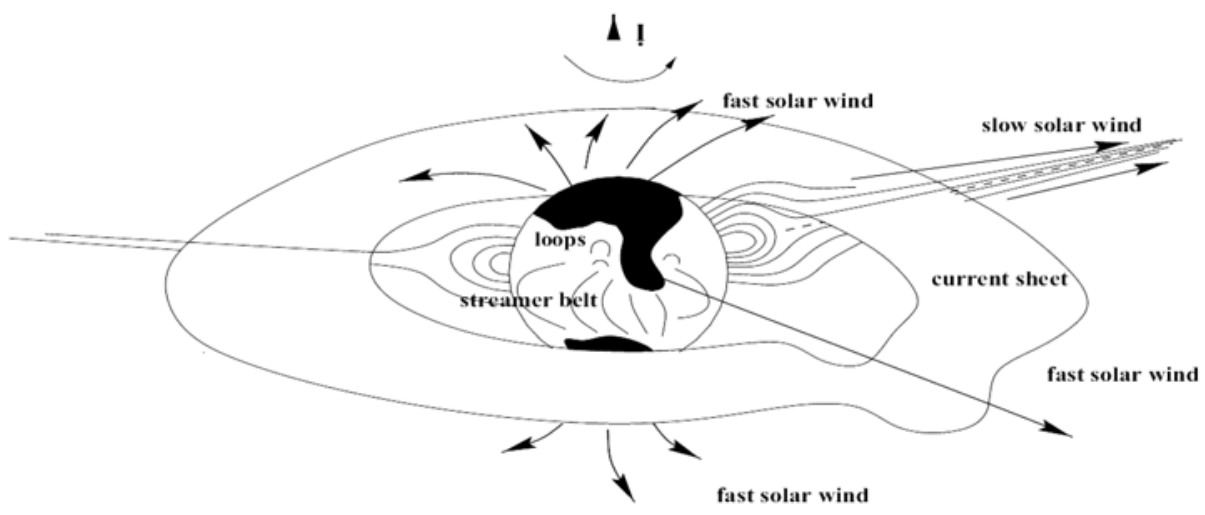

Fig. 11 Sketch of the corona/solar wind (coronal holes in black) at activity minimum. The lines ending on the solar surface around the equator represent magnetic field lines, those with an arrow the outflowing solar wind. (From Noci 2000) 


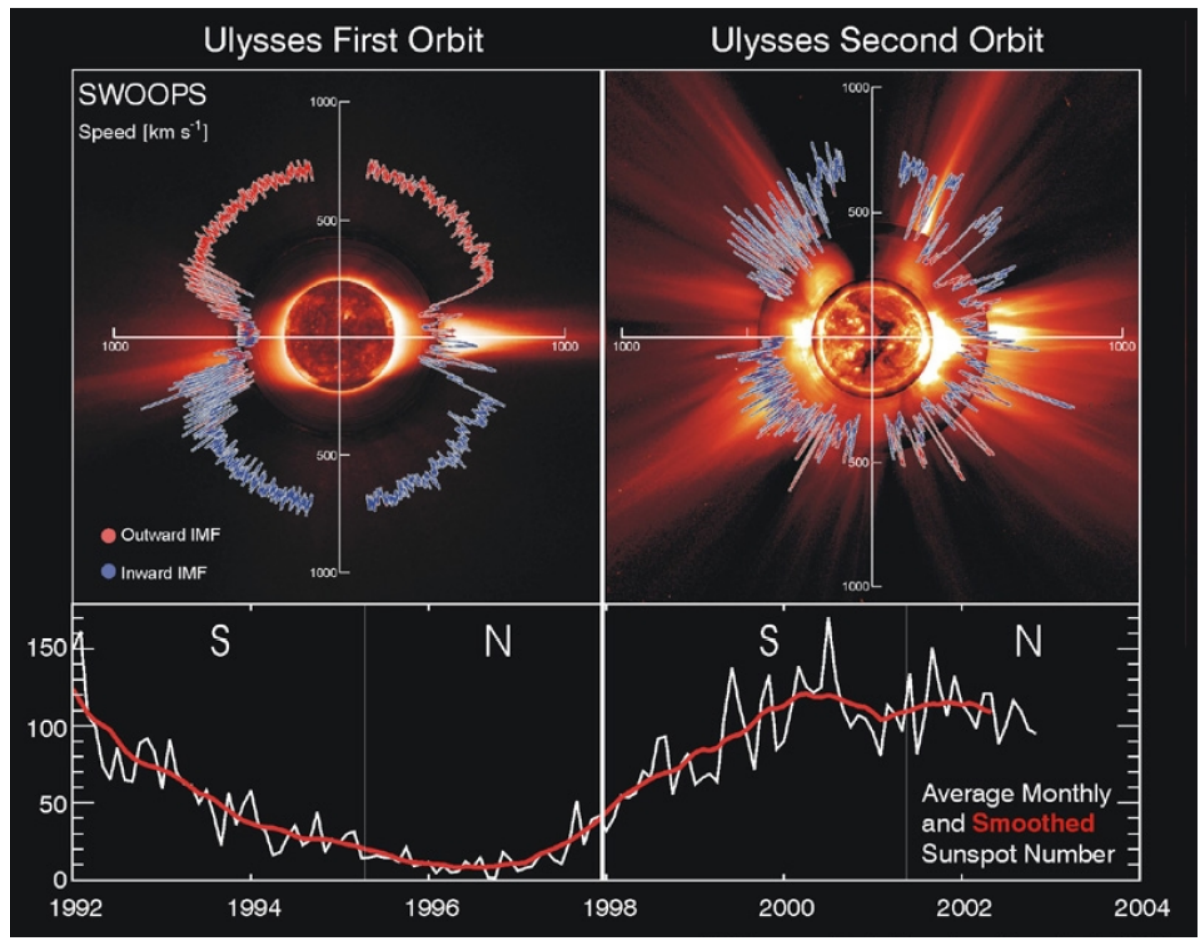

Fig. 12 Solar wind velocity as a function of latitude superimposed on EIT (SOHO), Mauna Loa K-coronameter and LASCO C2 (SOHO) images of the solar corona, for declining and minimum solar activity (left) and for maximum activity (right). Sunspot number in the lower panel. (Adapted from McComas et al. 2003)

\subsubsection{Scientific Models for the Solar Wind}

Fluid models Parker's model, which is the simplest fluid model (i.e. a model where the solar wind is treated as a fluid), was soon improved by Parker himself by dropping the $T=$ const. assumption. Other generalizations have been introduced in the following years, treating separately different plasma components (protons, electron, ions), introducing an explicit time dependence, etc.

These models have not been completely successful, however. Their main shortcoming is their impossibility to produce a solar wind speed reaching the high values observed in the fast solar wind (close to $800 \mathrm{~km} / \mathrm{s}$, Fig. 12), unless some ad hoc terms are introduced in the equations (e.g. terms representing energy and/or momentum deposition, without any indication of its physical cause).

Furthermore, to treat the solar wind plasma as a fluid is very questionable, given the low densities of the interplanetary space. (Most particle species have essentially no collisions above a few solar radii of heliocentric distance, departure of the electron velocity distribution from the Maxwellian shape is important, which makes invalid the use of transport coefficients, etc.)

Kinetic models The alternative is a kinetic treatment, which had been initiated by Chamberlain (1960), who found a solar wind speed, at the Earth orbit, of only $10 \mathrm{~km} / \mathrm{s}$. Once the observations showed that the wind was supersonic, kinetic treatments were abandoned. 
Interest in the kinetic models has revived later, starting with the work of Brandt and Cassinelli (1966). Further progress has been made in the following years (Jockers 1970; Lemaire and Scherer 1971; Maksimovic et al. 1997; Landi and Pantellini 2001).

Kinetic models study the behavior of the velocity distribution function of the electrons, protons and ions. Therefore they rely on the Boltzmann equation

$$
\frac{\partial f}{\partial t}+(\mathbf{v} \cdot \nabla) f+\left(\mathbf{a} \cdot \nabla_{v}\right) f=\left(\frac{\partial f}{\partial t}\right)_{c},
$$

where $f$ represents the density in phase space, $\nabla_{v}$ the gradient in velocity space, a the acceleration due to macroscopic forces and $(\partial f / \partial t)_{c}$ the temporal variation of $f$ due to collisions.

Since the electrons are much lighter than the ions, they would escape if were not for a polarization electric field, E. This field, called the Pannekoek-Rosseland field, pulls back the electrons and pulls up the protons. It is a component of $\mathbf{a}$ in the previous equation together with gravity. This was the field considered by Chamberlain. Later, the contribution of Brandt and Cassinelli (1966) was an improved determination of the level above which there are no collisions, and of the density decrease with heliocentric distance. Finally, Jockers (1970) and Lemaire and Scherer (1971) included the condition that the flux of escaping electrons should be equal to that of the escaping positive charges otherwise the Sun would charge itself.

Kinetic models are now able to obtain supersonic solar wind speeds in the interplanetary space. As far as densities and temperatures are concerned kinetic models are now in better agreement with the observations than fluid models.

Other models are the SWMF model (Toth et al. 2005), which produces a model of the ambient corona and inner heliosphere for the Carrington Rotation selected by the user in a 3D Cartesian grid (see Table 6) and the ENLIL model (Xie et al. 2004), which is a timedependent 3D MHD model of the heliosphere, that solves equations for plasma mass, momentum and energy density, and magnetic field, using a Flux-Corrected-Transport (FCT) algorithm (see Table 6). Another model provides the non-monotonic total potential for the protons, with a Lorentzian velocity distribution function (VDF) for the coronal holes (Lamy et al. 2003).

The Exospheric Solar Wind Model provides an exospheric model of the solar wind with only protons and electrons, the MAS model provides an MHD model of the solar corona in the range 1-30 solar radii, giving as output the solar coronal temperature, plasma density, pressure velocity and magnetic fields, and the WSA/PF+CS-IH model, able to determine the local solar wind speed (see Table 6 for the characterization of such models).

The Hakamada-Akasofu-Fry model is a de-facto operations model (HAF v2; Fry et al. 2007, and references therein). It is a "modified kinetic" model, as it kinetically projects the flow of the solar wind from inhomogeneous sources near the sun out into interplanetary space and adjusts the flow for stream-stream interactions as faster streams overtake slower ones. The MHD solutions integrate the equations of motion to obtain velocity and the kinetic model begins with the equations integrated twice to yield the fluid-parcel positions. The ambient solar wind is established by the model's initial conditions on the inner boundary. The event-driven component is represented by time-dependent boundary conditions when energy is fed at the inner boundary.

\subsection{Suprathermal Solar Particles}

Another aspect of solar weather is the release in the heliosphere of suprathermal particles. Such particles were named solar cosmic rays and they were identified as individual events in 


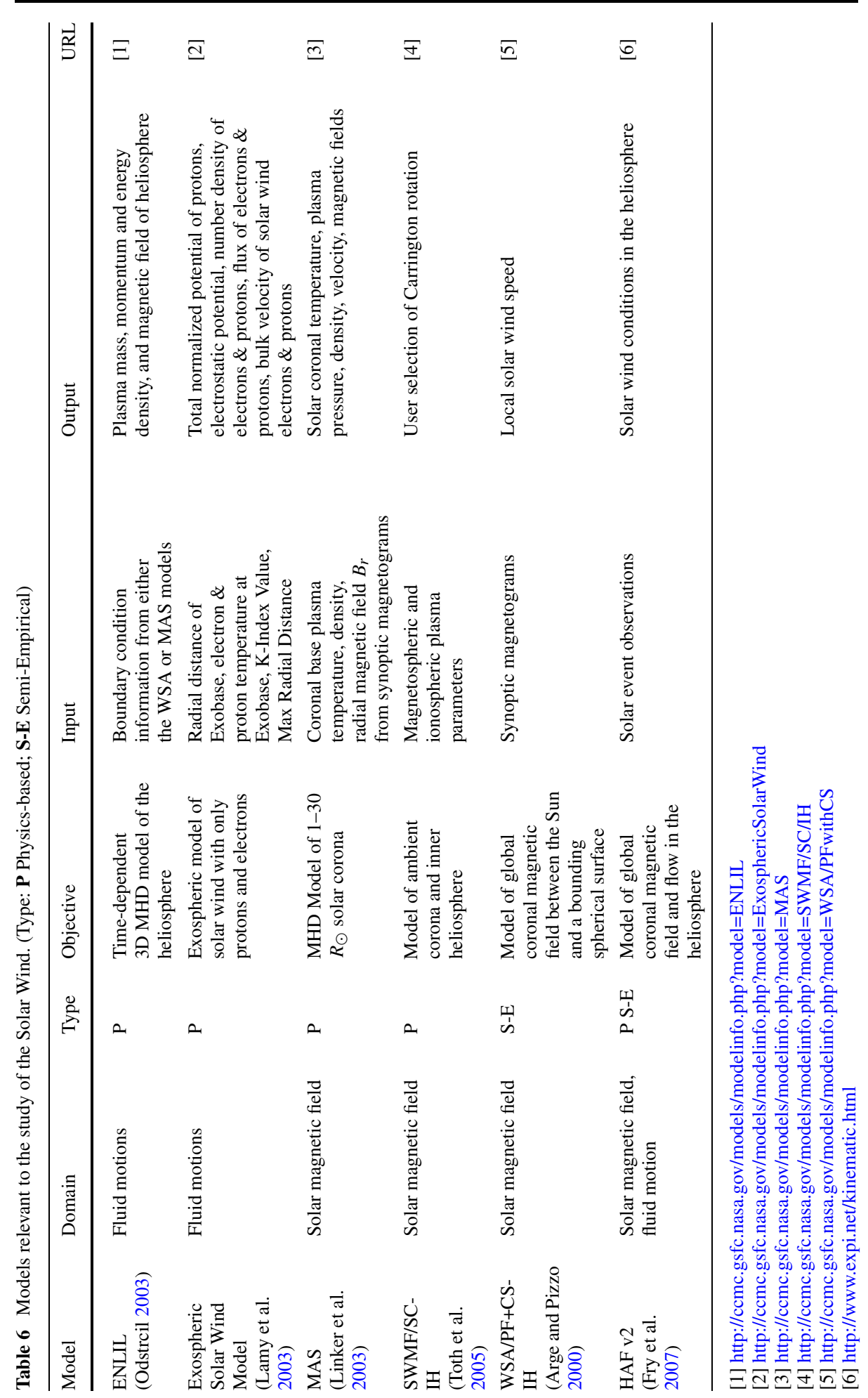


the recorded particle flux, first by ground-based terrestrial instruments (Forbush 1946) and after with those derived from the evolution of space research (on board balloons, satellites and space vehicles). The energies of these particles (which are composed by a high percentage of protons, even though other ions, electrons and neutrons are present) go from above the solar wind energy (few tens of $\mathrm{keV}$ ) to the relativistic ones (at least up to $\sim 30 \mathrm{Ge} \mathrm{V}$ ). Vainio (2009) summarized most of the relevant aspects related to this topic, particularly for what concerns the dynamics of the Earth's radiation environment. Here we concentrate our attention only on some basic concepts, which are key points for the development of particle simulation codes and models (see, for early reviews, Miller et al. 1997 and Pérez-Peraza 1998). We recall that synergies between solar, interplanetary and planetary measurements are necessary to solve problems related to the complete description of solar cosmic ray events, being the issues of particle production/acceleration on the Sun, escape/propagation, eventually re-acceleration, ion composition/charge states and 3D-flux decay in the heliosphere not completely clarified and modelled. Nevertheless, research focuses the attention on the source of solar cosmic rays by considering the magnetic topology changes in the solar atmosphere (see Fig. 2) and on the two interdependent phenomena at their origin: macroscopic processes (involving a large spatial scale) and microscopic processes (occurring within a small spatial scale).

Macroscopic phenomena are related to magnetic reconnection processes (magnetic merging, current loop coalescence, filamentary current sheets with multiple X-points, etc.) in active field regions with a certain degree of complexity, such as those originating flares (Sect. 4.2) and CMEs (Sect. 4.7). Several models propose that particle acceleration is mainly due to the action of the direct (or induced) electric fields (E; direct current or DC mechanism with a systematic monodirectional energy gain from a deterministic process) associated with the magnetic reconnection in the flaring region.

Available DC models consider the following topologies: - magnetic neutral current Xpoints and lines; - magnetic neutral current sheets; - magnetic neutral current layers. Such models depends on the $\mathbf{E}$ orientation to the $\mathbf{B}_{\text {local }}$ one (perpendicular and parallel DC fields). In particular, models considering $\mathbf{E} \| \mathbf{B}_{\text {local }}$ describe the source of particles in terms of (i) the double layer acceleration, (ii) the current interruption in force-free magnetic flux tubes and (iii) the runaway acceleration. These models are considered in quasi-static current structures and can be distinguish further in those having sub- or super-Dreicer fields (Dreicer 1959, 1960). Instead, using dynamic current layers, Haerendel (1994) explored, with good results, the capability of the field-aligned potential drop acceleration in solar flares, being the mechanism able to convert energy stored in magnetic shear stresses into kinetic energy.

Stochastic processes are also invoked for particle acceleration at the Sun, considering weak plasma turbulence conditions (i.e. "electrostatic modes" such as Langmuir waves, lower and upper hybrid waves, acoustic waves, whistler waves, cyclotron waves or "electromagnetic modes" in terms of Alfvén waves, fast and slow magnetosonic waves, among others; see Chen 1974). The performed studies require the interaction between waves and particles, i.e. a microscopic process involving a small-scale phenomenon. This implies that particles gain and lose energy in random small changes, but statistically there is a net gain of energy in time. When the Doppler resonance condition (which is tied to the wave dispersion relation $\omega(k)$ and the harmonic number $S$ ) is analysed for $S=0$ and $\omega$ less than the particle gyrofrequency, results led to identify the Čerenkov acceleration under diverse turbulence conditions (see columns 1 and 2 in the table reported by Pérez-Peraza 1998). For normal Doppler resonance $(S \geq 1)$ and greater or equal than the particle gyrofrequency the gyroresonant acceleration can work in the different turbulent conditions (see columns 1 and 3 in the table reported by Pérez-Peraza 1998, and the last column for limitations). Shortly, stochastic 
acceleration is possible on the Sun if a large wave spectrum can be assumed. In this case, electron acceleration is expected in the presence of electromagnetic waves, Langmuir waves or whistler waves, while acceleration of electrons and ions require at least Alfvén waves, magnetosonic waves or ion sound waves. Present scenario supports this understanding.

The third type of particle acceleration concerns the shock wave acceleration. In this case people distinguish between: - shock-drift acceleration (SDA mechanism) related to the shock presence in the corona, considered as a scatter-free region for the particles (an example is the first-order Fermi acceleration at fast shocks). Hence, the SDA mechanism is close to the DC mechanism; - diffusive-shock acceleration (DSA mechanism) associated with the shock passage through an enhanced turbulent/scattering region where particles are scattered many times forth and back across the shock layer (multiple shock encounters). DSA is closer to the case of stochastic acceleration, but in the latter case the plasma regime should be of the weak-wave turbulence type.

There is a large amount of literature on the topic that is impossible to report here (see Aschwanden et al. 2008, for a comprehensive review on progress in modelling solar structures, $\mathrm{CME} /$ shocks and solar energetic particles). Generally, to explain the different characteristics observed in solar particles events a continuous acceleration in one-phase (related to the DC mechanism-mainly impulsive events) was proposed for some cases and for others (gradual or two-component events) an intermittent acceleration with two or more acceleration phases (mainly associated with stochastic or shock wave accelerations) with possible particle trapping between them. Moreover, the so-called "thick target" models are proposed for solar regions with high density and long confinement time causing the particle trapping in closed configurations characterized by converging magnetic fields and a collisional regime (e.g. chromosphere and low corona), while "thin target" models apply in solar regions with low density and short confinement time, such as the high corona, where the magnetic field topology facilitates the free particle escape. A combination of both target classes is sometime also invoked as, for instance, a first particle acceleration in a thin target with particle escape in the interplanetary medium (prompt component), followed by a second component (delayed component) coming from the particle precipitation into an interaction region associated with a thick target. Or, conversely, a delayed component originating during the impulsive flare phase (but released later in the interplanetary medium as a consequence of an opening of the closed magnetic structures) and a prompt component produced afterward in the high corona (CME/filaments) but leaving immediately the Sun along the open field lines.

Finally, some of the above described mechanisms need a seed particle population which can be the solar wind itself or particle remnants from previous energy acceleration processes (for this reason it is believed that active regions producing a long series of flares are good candidates to be associated with solar relativistic particles).

\subsection{Solar XUV-EUV Models for Space Weather Applications}

Solar irradiance measurements and modelling efforts based on solar photospheric magnetic fields are extensively reviewed in Domingo et al. (2009), who considered the study of solar variations on time scales from days to the solar cycle. The authors pointed out how solar irradiance variability can be explained in terms of radiative effects of the photospheric magnetic activity, in turn related to the sub-photospheric convection as indicated by the most advanced magnetohydrodynamic simulations.

Here we concentrate on the solar irradiance in the ultraviolet range as one of the key parameters for space weather (Lathuillère et al. 2002) for which still very few continuous 
and spectrally resolved measurements exist. The irradiance must be measured from space but present detectors suffer from degradation and do not allow one to make long-term measurements.

The solar irradiance in the XUV-EUV-UV range is absorbed at Earth by molecular oxygen, atomic oxygen, ozone and molecular nitrogen. There are mainly three processes at work: ionization, excitation, and dissociation. Photoionization is mostly efficient above $150 \mathrm{~km}$, and filters the light down to about $80 \mathrm{~km}$ (see Belehaki et al. 2009). The main species involved are $\mathrm{O}_{2}, \mathrm{~N}_{2}$ and $\mathrm{O}$. Between 70 and $280 \mathrm{~nm}$, photodissociation of $\mathrm{N}_{2}$ and $\mathrm{O}_{2}$ becomes an important or predominant process; it filters the light down to low altitudes, typically $20 \mathrm{~km}$. The near ultraviolet (300 to $400 \mathrm{~nm}$ ) is mostly absorbed by the dissociation of ozone, whose efficiency peaks around $40 \mathrm{~km}$. This altitude is mainly of interest for classical weather and climate and, in spite of the importance of the phenomenon for human beings, is out of the scope of space weather purposes.

The impacts of the EUV and XUV fluxes on space weather through the atmospheric system are important for satellite drag and orbitography, for telecommunication and positioning and for the understanding of the relationships between space weather and climate.

The models currently used in space weather (Table 7) can be classified in several groups. The first one was raised before 2001. It uses extensively the Atmosphere Explorer data base (Hinteregger et al. 1973). It resulted in the fact that many present models deal with the wavelength segmentation proposed first by Torr and Torr (1979). The success of this approach can be ascribed to its simplicity and the existence of a set of absorption cross sections for each wavelength bin. There are two reference fluxes: one for active and one for quiet conditions. Other levels of activity are modelled by interpolating the decimetric radio index $f_{10.7}$. The set of data used to determine the flux has been gradually improved (Hinteregger 1981; Hinteregger and Katsura 1981; Torr and Torr 1985).

Tobiska (1991, 1993) and Tobiska and Eparvier (1998) developed a different model, called EUV, using a more extended data base. In comparison to the previous ones, this model retrieves the solar flux from the decimetric index and its 81 days average. The latest versions are called SOLAR2000 (or S2K) by their authors. They use new input parameters computed from a previous version of the code (Tobiska et al. 2000). They provide solar spectral irradiances and integrated solar irradiance proxies from 1947 to 2052. It includes complementary models to extend to the vacuum ultraviolet (VUV) (Tobiska and Bouwer 2006). The most recent code implementation is SIP (Solar Irradiance Platform) that produces the variable, full solar spectrum in assorted spectral formats for historical, nowcast, and forecast applications as well as an array of solar irradiance and thermospheric proxies (Tobiska 2008). SIP includes both SOLAR2000 and SOLARFLARE, a solar flare evolution prediction model (Tobiska 2005).

EUVAC (solar EUV flux model for aeronomic calculations) (Richards et al. 1994) is based on a reference flux that differs from the one used by Torr and Hinteregger, and relies on specific interpolation formula. EUVAC also adds physical constraints on the coronal flux. Its latest version, named HEUVAC (high-resolution solar EUV irradiance model for aeronomic calculations) (Richards et al. 2006), extends the EUV model below $5 \mathrm{~nm}$ and includes data from the SEE (Solar EUV Experiment) instrument on board TIMED (Thermosphere Ionosphere Mesosphere Energetics and Dynamics) (Woods et al. 2005).

Recently, autoregressive algorithms have been developed to forecast solar activity on time scales of 1 to 10 days (Lean et al. 2009). Following this model, quantitative assessment of solar activity observations and forecasts over 27 years (from 1980 to 2006) indicates that the chromospheric $\mathrm{Mg}$ index is superior to the coronal $f_{10.7}$ radio flux, both as a proxy for the day-to-day EUV irradiance variations that drive density changes and as an input to empirical models for density forecasts. 


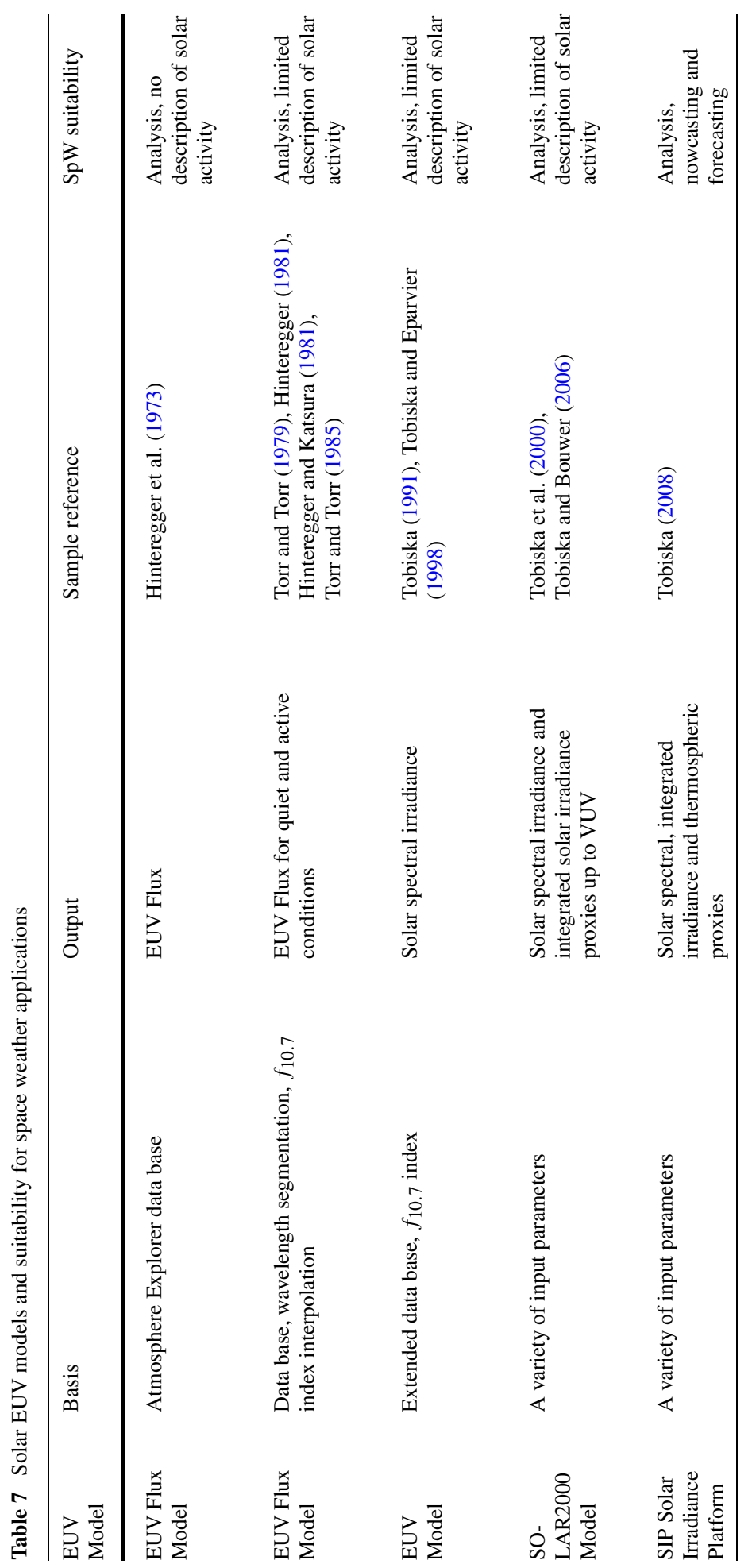




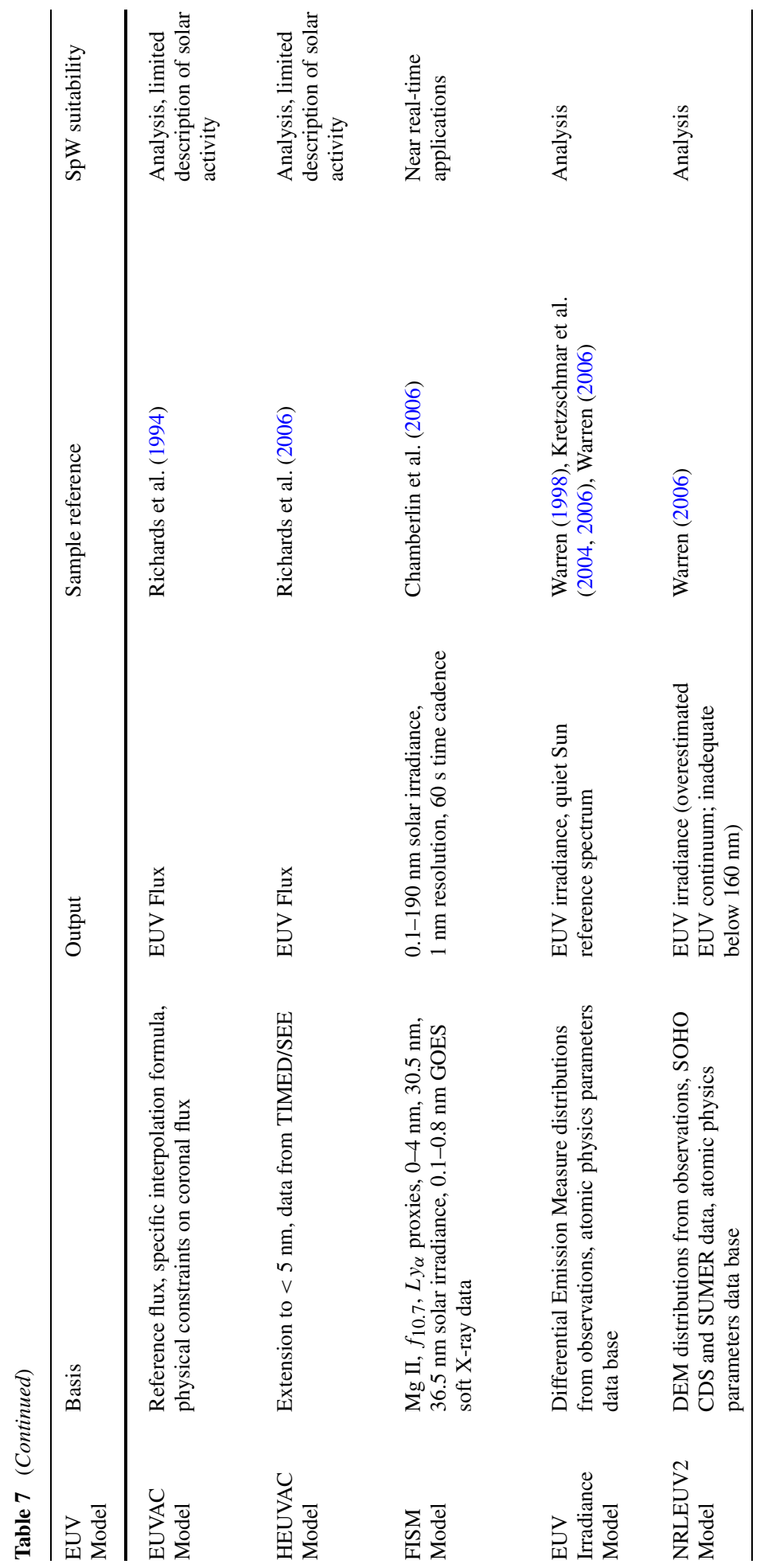


All these models are useful tools for space weather studies and are used in operations for tracking solar activity. Shortcomings of the models include uncertainties from using proxies to span the spectral irradiance data gaps and inability to represent all solar conditions (Dudok de Wit et al. 2005). All these models rely on one or a few indices that only partly describe the multiple facets of solar activity. Commonly used indices have limits as shown by Dudok de Wit et al. (2008) who demonstrated that no index is representative of the variability of the EUV spectrum at all wavelengths. Therefore, accurate forecasting is limited to the uncertainties of correlations between proxies and spectral irradiance in all of these models, whatever their (numerous) qualities are.

The second category of models uses additional inputs to reach better accuracy. SOLARFLARE (Tobiska 2005) uses GOES 0.1-0.8 nm flux to create a temperature vector that is used by a Mewe model (Mewe et al. 1985, 1986) subroutine to produce the timeresolved, 1-minute irradiance spectrum from $0-30 \mathrm{~nm}$ at $0.1 \mathrm{~nm}$ resolution. In addition, it incorporates a flare evolution empirical model to capture the time evolution of irradiances once a flare has initiated. It has been implemented operationally to provide flare-induced spectra for ionosphere models such as IFM (Ionospheric Forecast Model; a physics-based model of the ionosphere from 90 to $1600 \mathrm{~km}$, Schunk et al. 1997) which is at the core of GAIM (Global Analysis Integration and Modelling; http://gaim.unh.edu/). The Flare Irradiance Spectral Model (FISM) is based on data from TIMED. FISM is an empirical model that estimates the solar irradiance from 0.1 to $190 \mathrm{~nm}$ with $1 \mathrm{~nm}$ resolution, and with a time cadence of 60 seconds (Chamberlin et al. 2006). FISM can therefore model both eruptive events (for which very few accurate measurements exist) and long-term effects. Its inputs are traditional proxies $\mathrm{Mg}$ II, $f_{10.7}$, and $\mathrm{Ly}_{\alpha}$ and the irradiance in several bands $(0-4 \mathrm{~nm}$, $30.5 \mathrm{~nm}, 36.5 \mathrm{~nm}$ ) to model the daily component. FISM also makes use of soft X-ray measurements from GOES $(0.1-0.8 \mathrm{~nm})$ to model flares. This model is the first one that can be used for near real-time space weather operations.

The third category involves a radically different approach that has been investigated independently by two teams. Instead of relying on existing irradiance observations, the idea is to use Differential Emission Measure (DEM) distributions derived from spatially and spectrally resolved solar observations, full-disk solar images, and a data base of atomic physics parameters, to calculate the solar EUV irradiance (Kretzschmar et al. 2006; Warren 2006). These efforts have resulted in the definition of a quiet Sun reference spectrum and solar minimum irradiance observations (Warren et al. 1998; Kretzschmar et al. 2004). NRLEUV2 is the latest model developed by Warren (2006), which also includes data from the CDS and SUMER spectrometers on SOHO. Although the overall agreement with the observations is quite good, some discrepancies exist (Woods et al. 2005). The computed spectra overestimate the EUV continuum and cannot properly reproduce the observed irradiances below $160 \mathrm{~nm}$. Such discrepancies are inevitable as the underlying conditions are not all fulfilled: not all lines are optically thin, assumptions need to be made on the pressure, temperature and electron density profiles, relative abundances must be known, etc. (Kretzschmar et al. 2004). In spite of these limitations, models such as NRLEUV2 are valuable tools for research. Their relevance for space weather operations is as yet more questionable.

\subsection{Coronal Mass Ejections}

\subsubsection{Observation, Phenomenology and Basic Properties}

In the early 1970s the first space telescopes were developed through which imaging of the faint outer atmosphere of the Sun, the corona, became feasible apart from times of total solar eclipses. 

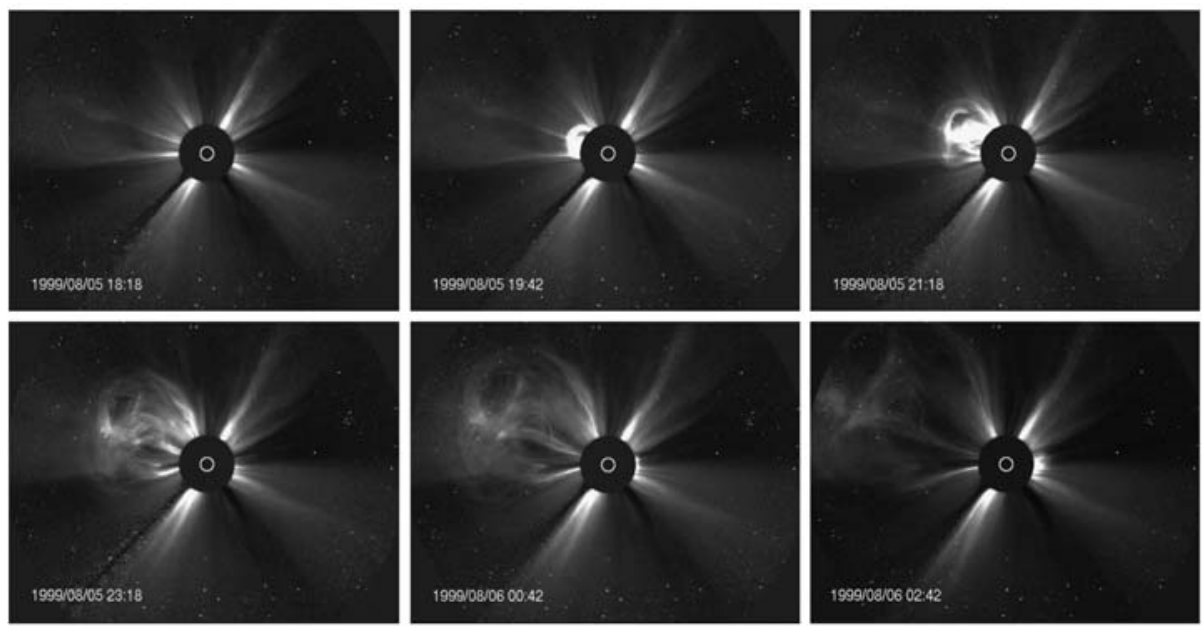

Fig. 13 A fast solar coronal mass ejection (CME) observed at the East limb on August 5, 1999 by SoHO/LASCO in the field of view from 6-30 $R_{\odot}$. The CME reached a speed of about $1000 \mathrm{~km} / \mathrm{s}$. Courtesy: SoHO/LASCO consortium

The images of these coronagraphs on board the OSO (Orbiting Solar Observatory) 7 satellite and on Skylab (Tousey 1973; MacQueen et al. 1974) surprisingly revealed the existence of huge outward expulsions of coronal matter. Such coronal transients, today commonly referred to as coronal mass ejections (CMEs), had remained undiscovered hitherto because the corona is $10^{6}$ times in intensity less bright than the visible solar disk and only observable from Earth during a total eclipse, which unfortunately takes only a couple of minutes not allowing one to watch large-scale dynamic phenomena. The light of the corona is mainly from photospheric light scattered at free electrons of the fully ionized coronal plasma and usually the Earth's atmospheric layers make it difficult to operate a sensitive enough ground-based coronagraph to detect some CMEs as in the case of the one installed at the Mauna Loa Solar Observatory (St. Cyr et al. 1999).

The LASCO (Large Angle Spectroscopic Coronagraph) instruments on board the SOHO satellite (Brückner et al. 1995) have recorded with unprecedented spatial and time resolution more than $10^{3}$ CMEs in solar cycle 23 (1996-2008) of various sizes, angular width and speeds. Figure 13 shows the occurrence of a typical three-part structured CME in the field of view (6-30 $R_{\odot}$ ) of the C3 LASCO coronagraph, consisting of a bright leading edge, dark void and trailing bright core (e.g., Cremades and Bothmer 2004). The centre of the CME is located at a position angle (PA) of $+45^{\circ}$, as measured positively counterclockwise from the Sun heliographic North pole. By tracking the centre of the leading edge the speed can be estimated as $\sim 1000 \mathrm{~km} / \mathrm{s}$.

The trailing bright core of the CME is commonly interpreted as erupting prominence material which is likely an oversimplified consideration because of the complexity of the prominence cavity system and projection effects apparent in coronagraph observations (Fuller et al. 2008). The onsets of CMEs, especially that of large and fast CMEs, are usually accompanied with flaring EM-radiation at X-ray, EUV and sometimes even $\gamma$-ray wavelengths (Aschwanden 2004, p. 34). The CME kinetic energy of $10^{23}$ to $10^{24} \mathrm{~J}$ is comparable to that emitted through flaring radiation. It seems obvious that the violent launch of a $\mathrm{CME}$ causes magnetic fields of opposite polarities to reconnect quickly and causing sporadic EMradiation in the form of flares. This can happen well even after launch (Tripathi et al. 2004). 
Table 8 Basic characteristics of solar coronal mass ejections based on space-borne coronagraph observations (Bothmer and Zhukov 2006)

\begin{tabular}{ll}
\hline Speed & $<300->3000 \mathrm{~km} / \mathrm{s}$ \\
Mass & $5 \times 10^{12}-5 \times 10^{13} \mathrm{~kg}$ \\
Kinetic energy & $10^{23}-10^{24} \mathrm{~J}$ \\
Angular width & $\sim 24^{\circ}-72^{\circ}$ \\
Daily occurrence frequency & $\sim<1-\sim>4$ (sol. min.-sol. max.) \\
\hline
\end{tabular}

In a recent study Zhang (2001) analysed in great detail the temporal and physical relationship between CMEs and flares and found that the kinematic evolution of those CMEs associated with flares shows a three phase development: in the initiation phase the CME slowly rises for a time period of several tens of minutes followed by the onset of the X-ray flare and the impulsive acceleration phase of the CME until the acceleration ceases and the CME starts propagating farther out at constant speed and by maintaining its angular width.

CMEs roughly carry $5 \times 10^{12}$ to $5 \times 10^{13} \mathrm{~kg}$ of matter into space with an angular width of about $70^{\circ}$ (e.g., Vourlidas et al. 2002). Their speeds are often fairly constant over the first couple of solar radii (e.g., St. Cyr et al. 1999, 2000) and range from a few tens of $\mathrm{km} / \mathrm{s}$ to speeds up to $3000 \mathrm{~km} / \mathrm{s}$. During low solar activity a typical CME is seen every couple of days whereas at maximum the daily rate can exceed 10 or more. The basic parameters are summarized in Table 8.

The source regions of CMEs can be traced back to bipolar regions of the photospheric magnetic field, either to active, but also to quiescent ones (Cremades and Bothmer 2004; Tripathi et al. 2004). Even $1600 \mathrm{~km} / \mathrm{s}$ fast CMEs can originate from quiescent extended bipolar regions, without associated flaring (Bothmer and Zhukov 2006, Fig. 3.62).

For more extended reviews on the physics of CMEs the reader is referred to the specific books edited by Crooker et al. (1997) and Kunow et al. (2006).

\subsubsection{CME Modelling}

3-D structure of CMEs, projection effects and halo CMEs Cremades and Bothmer (2004) found CMEs usually to originate in coronal regions overlying bipolar photospheric fields of opposite magnetic polarity. Based on this finding they could compare the SOHO MDI (Michelson Doppler Imager) magnetograms in detail with the CME white-light characteristics of typical three part structured CMEs imaged with LASCO C2 and develop the basic scheme shown in Fig. 14.

If one assumes that the average orientation of the neutral lines separating bipolar regions as CME sources follows Joy's law, the characteristic white-light shape of a CME seen in the field of view of a coronagraph can be explained naturally through the basic scheme presented in Fig. 14. CMEs originating from the visible solar disk are seen at the East limb in cross-section and sideways at the West limb. The scheme reverses for CMEs originating at the back-side of the Sun. Thernisien et al. (2006) have successfully reproduced the whitelight pattern for the CMEs analysed by Cremades and Bothmer (2004) through a graduated cylindrical shell (GCS) model.

The apparent profile of an individual CME may differ more or less from the basic scheme presented in Fig. 14 because of the solar variability of the fundamental underlying parameters-for example, many neutral lines are not straight but have rather complicated topologies, especially in active regions. Close-by emerging bipolar regions can form multi-polar configurations, the source region lengths can shrink and hence the length of the magnetic flux-rope axis decreases. 

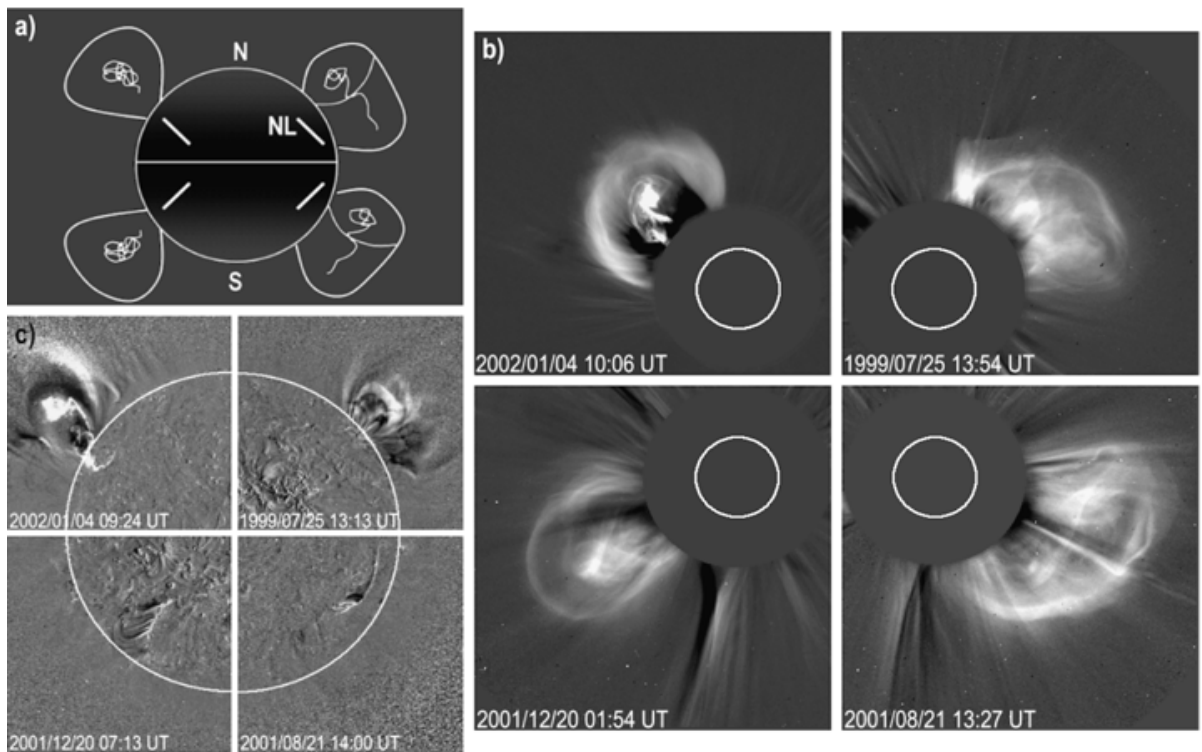

Fig. 14 a) Basic scheme showing the extreme cases of CME projection for front-side events. NL stands for neutral line, i.e. the polarity inversion line separating the two opposite photospheric magnetic polarities. b) Four projected CMEs seen by SoHO/LASCO C2 representing the scheme. c) $19.5 \mathrm{~nm}$ signatures identifying the source regions of CMEs. For the Northern events eruptive signatures were selected while for the Southern ones post-eruptive features are shown. From Cremades and Bothmer (2004)

Contrary to the white-light structure of the CMEs shown in Fig. 14, events originating from the solar disk appear as halo CMEs (Howard et al. 1982). Figure 15 shows a typical front-side halo, i.e. one with associated activity on the solar disk in form of post-eruptive loops, disappearing filaments (erupting prominences) or flare signatures. This large CME was associated with a disappearing filament and originated from a rather small region on the Sun.

The basic CME models The major CME models have been reviewed e.g. by Klimchuk (2001) and Forbes et al. (2006). Commonly the models are classified into five categories in terms of mechanical analogues (see Table 9). The thermal blast model bases on greatly enhanced thermal pressure as CME driving force, produced by a flare and similar to a bomb explosion (e.g., Dryer et al. 1979; Wu 1982). This model seems inconsistent with the observation of CME occurrences without flares. The so-called dynamo model implies a rapid generation of magnetic flux by real-time stressing and/or kinking of the magnetic field comparable to the compression of a spring by an external force (Klimchuk 1990; Török et al. 2003; Blackman and Brandenburg 2003). On the Sun this could be generated for example by the rapid displacement of the footpoints of a coronal loop system. Simulations by Krall et al. (2000) use injection of magnetic flux as driving mechanism in which pre-existing fields become twisted, new ring-shaped field lines rise upward in the corona while becoming detached from the photosphere and new arch-shaped field lines emerge into the corona while staying anchored at their photospheric footpoints. Chen (1996) and Roussev et al. (2004) amongst others assume a priori that the structure of a CME is that of a magnetic flux rope in agreement with the findings by Cremades and Bothmer (2004) on the 3-D structure of CMEs derived from SOHO/LASCO 


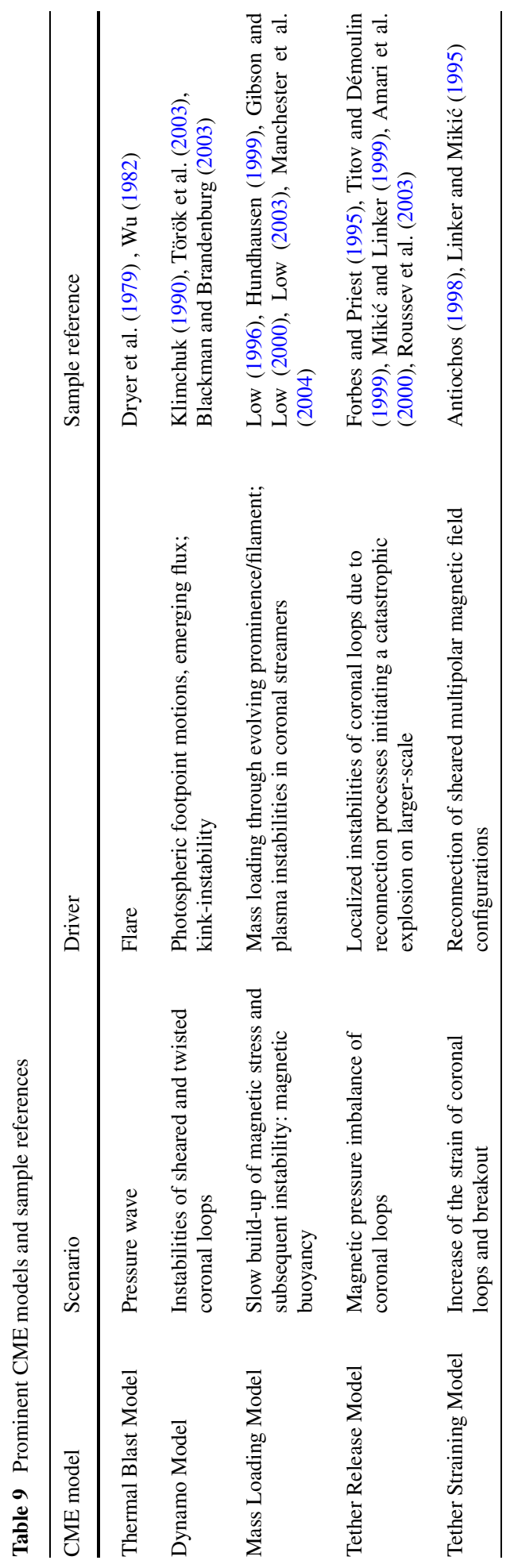



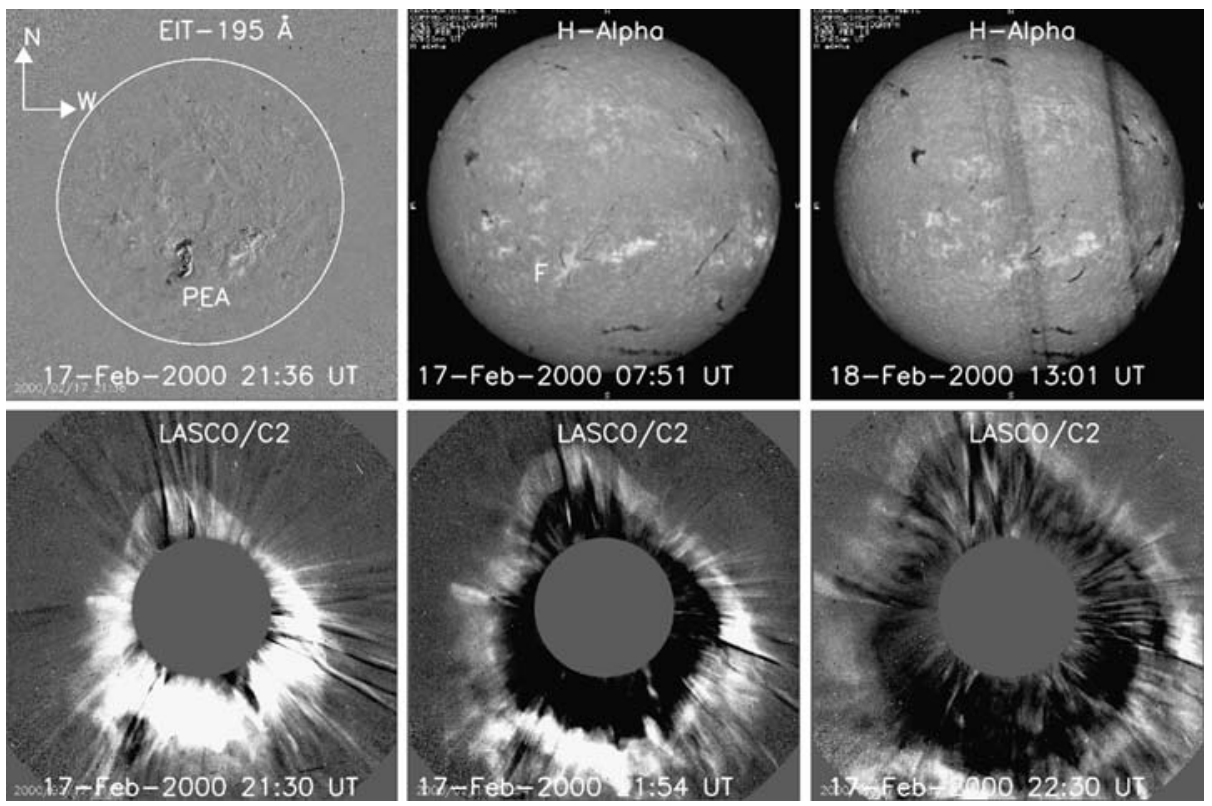

Fig. 15 Top left: SoHO/EIT $19.5 \mathrm{~nm}$ image showing the post-eruptive arcade which formed after the front-side halo CME observed by LASCO/C2 on 17 February 2000. Middle and right images: $H_{\alpha}$-images from the Paris/Meudon Observatory showing the disappearance of the associated filament. Bottom images: $\mathrm{SoHO} / \mathrm{LASCO} / \mathrm{C} 2$ images showing the near-Sun development of the halo CME. The speed of the CME was about $600 \mathrm{~km} / \mathrm{s}$. Note the asymmetry of the halo in the NE to SW direction. From Tripathi et al. (2004)

observations. The eruption of the flux rope occurs in response to the injection of magnetic flux into the rope. It is however unanswered to date whether the flux rope emerges from below the photosphere (Rust and Kumar 1994) or if it is formed above the photosphere (e.g., Gosling et al. 1995). Contrarily, other authors (e.g., Forbes and Priest 1995; Shibata 2001) assume a loop arcade-like structure. Emerging flux however could be a driving force lifting the CME mass upward as well as helical field configurations (Blackman and Brandenburg 2003). Three other generic CME models are based on the concept of energy storage and release in which magnetic stress is increased before the eruption, e.g. through mass loading (e.g., Low et al. 2003). Like a weight compressing a spring which explosively uncoils when the mass is removed. Mass loading could be achieved by a slowly rising prominence. In the tether release model (e.g., Forbes and Priest 1995; Titov and Démoulin 1999; Roussev et al. 2003) magnetic dominated systems of coronal loops involve a balance between the upward-directed force of magnetic pressure and the downward-directed force of magnetic tension. Tethers are the field lines that provide the tension in analogy to ropes that hold down a buoyant balloon. Emergence or cancellation of magnetic flux could lead to break-off of the tethers as proposed by several authors (e.g., Mikić and Linker 1999; Amari et al. 2000) and matching the observational photospheric magnetic field characteristics of the CME source region events studied by Bothmer and Tripathi (2007). In the tether straining model the strain on the tethers is gradually increased until they brake as introduced in the breakout models by Antiochos (1998) or Linker and Mikić (1995). The breakout is caused by two adjacent arcades of a quadrupolar field configuration in which one arcade is continuously sheared and reconnecting is initiated finally leading to the breakout of the CME loops. The different scenarios and references are summarized in Table 9. It should 
be noted that this section has focused on the standard type of CME, but that coronal jets and plasma blobs on smaller scales are described in terms of the so-called standard model and its variations which bases on 2D X-point reconnection scenarios (e.g., Tsuneta 1997; Shibata 2001).

\subsubsection{Evolution of CMEs into Interplanetary Space}

Typical CME observations by coronagraphs can be adequately described through magnetic flux-rope models (e.g., Cremades and Bothmer 2004; Thernisien et al. 2006). However, the evolution of them from the near Sun through the interplanetary medium at Earth's orbit is still a rather unexplored field compared to the wealth of solar remote sensing observations.

The solar wind structure and coronal mass ejections in the solar wind, commonly referred to as interplanetary CMEs or ICMEs, have been remotely sensed for the first time in a premature way in the inner heliosphere through the photometers on the two Helios spacecraft, designed to measure the zodiacal light, at distances between 0.3 and 1 AU (e.g., Webb and Jackson 1990; Webb et al. 1993; Jackson et al. 1994) and through the method of interplanetary scintillations (e.g., Hewish and Woan 1995). As heritage of the Helios photometer a more sophisticated white-light imager, the Solar Mass Ejection Imager (SMEI), has been developed and is currently operating on the US Coriolis satellite launched in 2003 (Webb et al. 2006).

In observations from Earth's orbit CMEs directed towards the Earth appear as halo whitelight features with corresponding source regions on the visible solar disk (e.g., Howard et al. 1982; Tripathi et al. 2004). Due to the projection effects inherent in the Thomsonscattered white-light data, it is difficult to obtain reliable speed estimates for the CMEs and extremely difficult to track them from the Sun to Earth. To date the first direct observations of CMEs all along the Sun-Earth line from the low corona to the Earth's magnetosphere are currently being analysed as provided by the unprecedented observations from the SECCHI (Sun Earth Connection Coronal and Heliospheric Investigation) on board the two NASA STEREO spacecraft launched in 2006 which image the Sun-Earth system from two vantage points leading and trailing Earth's orbit around the Sun (Howard et al. 2008; Davies et al. 2009). The STEREO observations will provide new insights into the 3D structure of CMEs and their evolution in the heliosphere which can directly be compared with existing models and simulations.

A good review on this topic can be found as section 3 by Siscoe and Schmidt in the CME modelling and theory summary by Forbes et al. (2006). Chen (1996) and Chen and Krall (2003) has modeled the evolution of a CME as a flux rope into the interplanetary medium in which satellites indeed detect large-scale magnetic flux-rope structures, referred to as magnetic clouds (e.g., Burlaga et al. 1982; Bothmer and Schwenn 1998). An archetype interplanetary CME observed by the ACE spacecraft in the L1 orbit ahead of Earth's magnetosphere is shown in Fig. 16. It caused one of the largest geomagnetic storms in solar cycle 23 in July 2000 because of its speed and southward-directed field associated with its internal magnetic flux-rope structure (Bothmer 2003; Bothmer and Zhukov 2006). As was the case for the CME shown in Fig. 16, faster than the ambient solar wind propagating CMEs have been found to be the driver of shock waves in the interplanetary medium (Sheeley et al. 1985; Bothmer and Schwenn 1996). The rotation of the magnetic field vector over a time interval of about one day is typical of magnetic flux-rope type CMEs at 1 AU (e.g., Bothmer and Schwenn 1998). Magnetic cloud type CMEs have typical radial sizes of $0.24 \mathrm{AU}$ and expand proportional with increasing distance from the Sun roughly proportional to $R^{0.8}$ (R in AU) causing the proton density to decrease roughly proportional to $R^{-2.4}$ (Bothmer and Schwenn 
Fig. 16 The ICME observed on July 15/16, 2000 by the WIND spacecraft. Data gaps are substituted with data from the Geotail satellite. The interplanetary shock ahead of the ICME is labeled by a solid line, dashed lines mark the boundaries of the ICME itself. Within the magnetic cloud type ICME, the magnetic field direction rotated from South to North, being directed Eastward at its centre. The ICME is of type SEN, i.e. having left-handed magnetic helicity. Displayed solar wind parameters from top to bottom: Magnetic field magnitude $B$, polar and azimuthal angles $\theta_{B}$ and $\phi_{B}$, solar wind speed $V$, proton density $N_{p}$ and thermal speed $V_{\text {th }}$. Courtesy:

Berdychevsky, from Lepping et al. (2001)

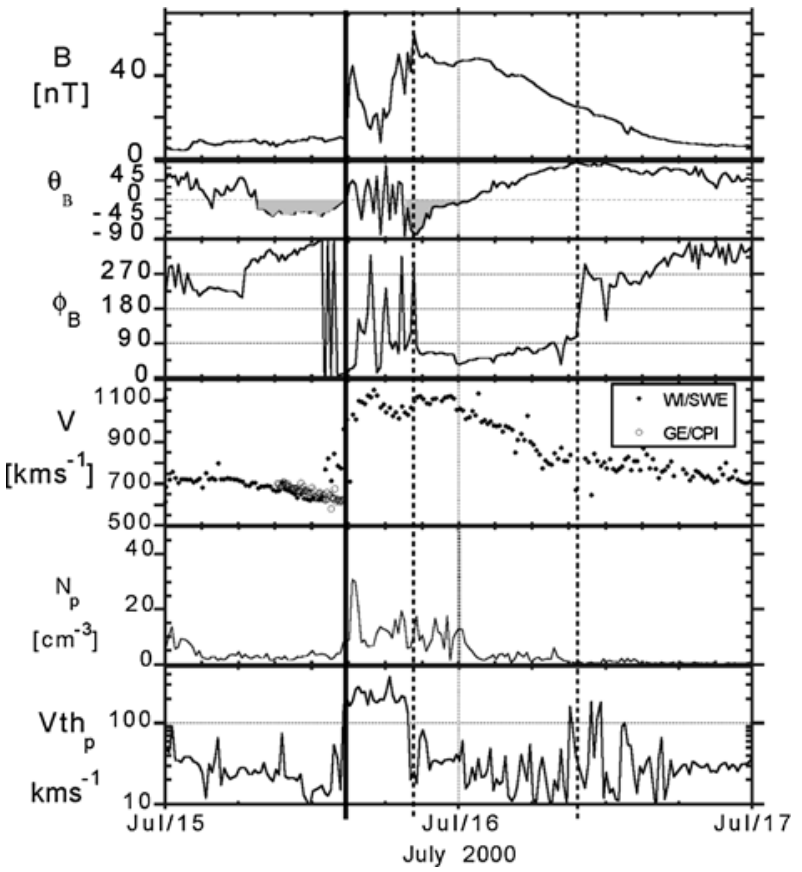

1998). Many magnetic clouds type CMEs can be modeled adequately through large-scale cylindrical force-free MHD configurations as introduced by Goldstein (1983) and elaborated by Lepping et al. (1990) and Bothmer and Schwenn (1998). The July 2000 CME shown in Fig. 16 matches the pattern for magnetic flux-rope types presented in Fig. 17 in which the internal magnetic structure of such CMEs depends on the magnetic field properties of its solar source region and the hemispheric helicity rule (Bothmer and Rust 1997). It should be noted that deviations from the scenario presented in Fig. 17 can be easily expected at times of higher solar activity when bipolar fields emerge in the solar photosphere in close proximity and the structure of active regions can become very complex.

\subsubsection{Predicting Geo-effective CMEs and Future Perspectives}

No model currently exists that can predict accurately the onset of CMEs at the Sun though some authors have claimed to have found possible hints (e.g., Thalmann and Wiegelmann 2008). It is obvious that the emergence of magnetic flux in the photosphere, at the different spatial and time scales, is a crucial process and the basic origin of activity but there is no quantitative understanding yet to predict the onset and speed of a CME based on solar parameters such as a normalized magnetic flux per source region volume, flaring intensity, intensity and energy of accelerated particles, amongst other parameters. So far we can only track bipolar regions as potential CME sources from the Sun's East- to West-limb and monitor CME onsets in white-light and the source region activity at EUV wavelengths (Tripathi et al. 2004) as well as monitoring changing photospheric flux which can change on time scales of minutes. However, it appears that East-limb CMEs are not the biggest threats for space weather at geospace because of the missing head on direction of these CMEs as well as the lack of magnetic connection to its solar source region, not favoring radiation hard particle events associated with it. Once a CME is detected through space-borne coronagraphs, 


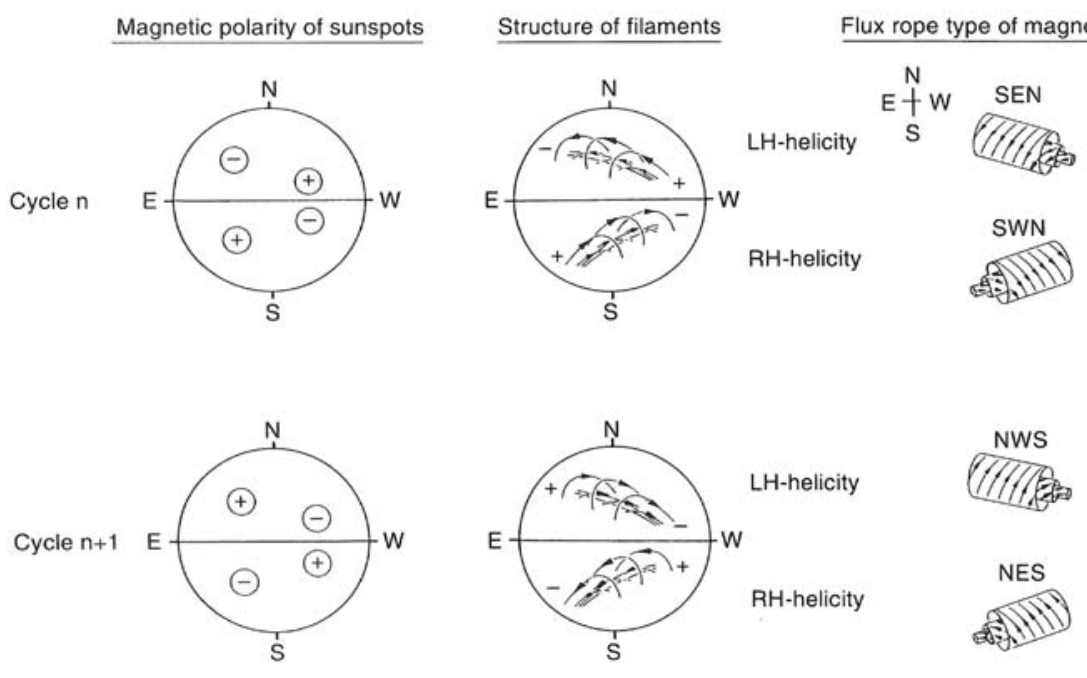

Fig. 17 Basic scheme showing the solar cycle dependence of the magnetic field structure of filaments at the Sun and that of the corresponding magnetic clouds (MCs) in the interplanetary medium. Note that for simplicity the MCs are shown oriented horizontally with respect to the ecliptic plane and that a cycle overlap is not taking into account as well as the possibility of photospheric magnetic field regions that reveal a more complex field configuration, i.e. e.g., quadrupolar regions. From Bothmer and Rust (1997) and Bothmer and Schwenn (1998)

which appear as a real need as solar space weather monitors, it has been so far difficult to measure the real speed of such a halo CME, as shown in Fig. 15, to forecast its arrival at Earth's orbit because of the projection effects (e.g., Cremades and Bothmer 2004) and to quantify its geo-effective parameters (i.e., the speed, intensity and duration of associated Southward-directed fields) in advance. In fact, the CME could interact more or less strongly with the ambient solar wind flow in the inner heliosphere, leading to more or less compression or expansion effects and acceleration or deceleration. The internal structure of the CME's magnetic field can often be directly deduced from the magnetic configuration of the CME source region as shown in Fig. 17, but a lot of research is still required to elaborate a reliable prediction system. Schwenn et al. (2005) have developed a relationship between the CME's travel time $\left(T_{\text {tr }}\right)$ to $1 \mathrm{AU}$ and its expansion speed $\left(V_{\text {exp }}\right)$ in the SOHO coronagraphs field of view according to which $T_{\text {tr }}=203-20.77 \ln \left(V_{\exp }\right)\left(T_{\text {tr }}\right.$ in hrs, $V_{\text {exp }}$ in $\left.\mathrm{km} / \mathrm{s}\right)$. The data currently received by the NASA STEREO mission, which consists of simultaneous observations from two satellites in heliosynchronous orbits that drift apart from the Sun-Earth-line by $22^{\circ}$ per year in opposite directions as described in detail in "The STEREO Mission", ed. by C.T. Russell (Russell 2008) allow for the first tracking of CMEs from Sun to Earth and will allow one to prove or reject existing theories on CME initiation, 3D structure, evolution and propagation. The new results will be doubtless a quantum step for space weather research and predictions.

A step forward in this context is represented by the development of a software package for the automatic detection and tracking of CMEs from SOHO/LASCO coronal images (Robbrecht and Berghmans 2004; http://sidc.oma.be/cactus/). Operated at the SIDC (Solar Influences Data Analysis Center, Bruxelles, Belgium), it provides lists of events, with principle angle, angular width and velocity estimation for any detected CME. Hence it can be used as an operational tool for space weather applications. 
Recently, a significant step forward towards the development of a reliable automated CME prediction system has been provided by Qahwaji et al. (2008), who conceived a machine learning system based on Cascade Correlation Neural Networks (CCNN) and Support Vector Machines (SVM) to analyse CME-flare associations and to predict, from the input of a flare's properties, if the flare is likely to initiate a CME. Flare events from the NGCD flares catalogue and CME events from the SOHO/LASCO CME catalogue are used to associate X- and M-class flares with CMEs based on timing information. This information is then fed to the machine learning system to create a set of rules to be used for the automated prediction of CMEs.

\subsection{Geoeffectivity of Solar Radio Bursts}

Solar radio bursts (SRBs) are a variety of electromagnetic signatures of thermal and nonthermal plasma processes occurring mainly in the chromosphere and corona, triggered e.g. by changes in the magnetic topology by newly emerging flux, magnetic reconnection in flares, propagating particle beams and shocks (see e.g. the reviews by Dulk 1985 and Bastian et al. 1998). Hence SRBs represents effective proxies of physics processes involving magnetic energy release, such as, but not exclusively, in flares, particle acceleration and emission of electromagnetic (em) radiation outbursts. Furthermore, the emission and propagation conditions of em waves in the radio domain are related to the plasma density and homogeneity and to the magnetic field characteristics both at the source and in the background plasma during the propagation, making SRBs suitable proxies not only for the generation processes but for the ambient plasma as well. Refined modelling and observations of SRBs provide relevant information on the source processes of solar weather and a key role for the applications of solar radiophysics to space weather will be played by the new generation radio telescopes like FASR (Frequency Agile Solar Radiotelescope) (Gary and Kellereds 2004) and LOFAR (LOw Frequency ARray) (e.g. Mann 2005), capable to track solar radio sources respectively at high and low frequencies, i.e., in the solar corona and in the interplanetary medium where e.g. CME are originated and where they propagates and radioemits.

Besides being effective plasma probes, various studies indicate that SRBs can act as direct sources of space weather effects by interfering wireless communications (see for a review e.g. Gary et al. 2005; Goodman 2005; Lanzerotti et al. 2005; Lanzerotti 2007; Messerotti 2008, and references therein) and Global Positioning Systems (GPSs) (see e.g. Klobuchar et al. 1999; Chen et al. 2005).

In fact, upon the geometrical constraints that the position of the Sun in the sky must lie in the antenna lobe of the receiving and repeater systems and that the SRB radio flux density exceeds certain estimated threshold levels (1000-8000 SFU (Solar Flux Units) in the 900$3000 \mathrm{MHz}$ range), the SRB-associated increase in the radio noise can cause the degradation of the communication quality up to the loss of mobile phone-cell repeater linking. This can be statistically estimated to occur with a time cadence dependent on the solar cycle phase, i.e., on the average once every 3.5 days at solar maximum and every 18.5 days at solar minimum (Bala et al. 2002; Nita et al. 2002, 2004; Lanzerotti et al. 2002).

Similarly, the decrease in Signal-To-Noise ratio caused by an intense right-hand circularly polarized (as the GPS antennas) SRB can cause the loss of signal lock in a GPS receiver, which can fail to produce a navigation solution (Chen et al. 2005; Cerruti et al. 2006). The failure of the sunlit high-precision GPS for more than 10 minutes during a solar flare on December 6, 2006 was investigated by Afraimovich et al. (2007, 2008). The associated SRB was characterized by a radio flux density of $10^{5} \mathrm{SFU}$ and peak flux density of $10^{6} \mathrm{SFU}$ despite that it occurred at the solar minimum. 
Finally, we mention the effect of SRBs on downlink satellite communications that has been pointed out by Kennewell (1989). Satellites in equatorial orbit are aligned with the Sun at the equinoxes and the satellite communications can be disrupted by the increase in radio noise caused by a SRB. In fact, as the solar radio emission raises with frequency, at $4 \mathrm{GHz}$ it can exceed by $20 \mathrm{~dB}$ the typical signal emitted by a satellite TV transponder. Such a signal interference only occurs once a day for any satellite and it lasts less than 8 minutes, but interferences lasting up to 30 minutes are observed dependent on the receiving antenna system characteristics.

In this framework, monitoring SRBs for nowcasting purposes requires dedicated solar radiopolarimeters operating in real-time, capable of providing accurate high time resolution measurements of radio flux density and polarization, such as, e.g., the Trieste Solar Radio System operated in Italy by the INAF-Astronomical Observatory of Trieste (TSRS; http://radiosun.oats.inaf.it/), which is compliant with the requirements for space weather applications and has been successfully used in wireless communication interference analysis (Afraimovich et al. 2008; Messerotti 2009). Figure 18 shows a potentially geoeffective SRB observed on April 15, 2001 by TSRS at both 1420 and $2695 \mathrm{MHz}$, which saturated the TSRS receivers (the NGDC catalogue reports 48,000 SFU at $2800 \mathrm{MHz}$ ). This SRB was associated with an X14/2B solar flare peaking at 13:50 UT, which originated also a solar proton event and resulted in ground level effects.

Prediction techniques for the radio effectivity of SRBs are beyond the present knowledge and only nowcasting can be successfully provided based on real-time solar radio measurements. Due to the fragmentary knowledge on SRB triggering and evolution as well as on possible precursors, it is expected that a viable approach in setting up a prediction system should be based on a combination of artificial neural networks and/or expert systems as done for flares and CMEs.

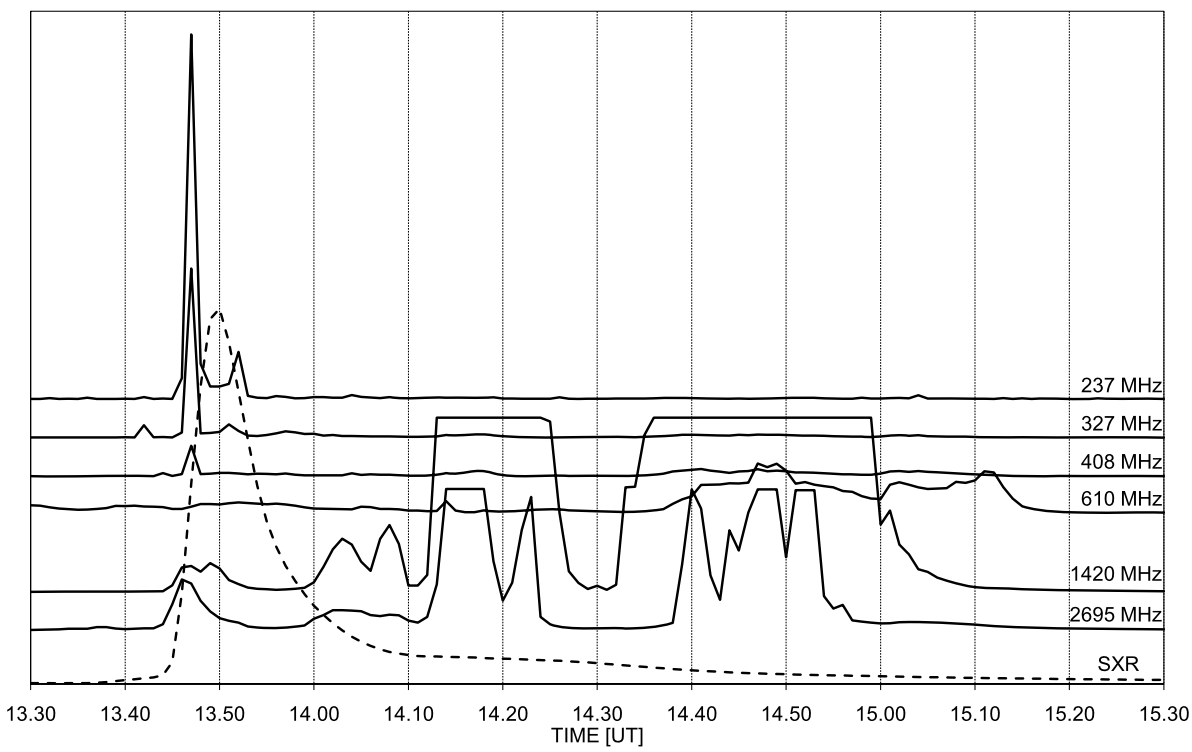

Fig. 18 Total solar radio flux density measured by the Trieste Solar Radio System at 237, 408, 610, 1420 and $2695 \mathrm{MHz}$ on April 15, 2001 during the evolution of an X14/2B flare, whose GOES SXR light curve is superimposed (vertical axis in arbitrary units). The radio flux density level of decimetric outbursts was so high that the 1420 and $2695 \mathrm{MHz}$ receivers exceeded the saturation level for tens of minutes 


\section{Summary and Conclusions}

We reviewed the present knowledge on key drivers of solar weather and mid-term solar climate by considering a selection of relevant physics- and statistics-based scientific models as well as a selection of related prediction models, in order to provide an updated operational scenario for space weather applications.

Solar-dynamo models, such as e.g. flux-transport and local dynamo models, can reproduce many observed features of the solar cycle, but not all of them, such as the differences in activity level in each solar cycle, the different periodicities and the long minima periods, and the slow increase in time of the interplanetary magnetic field. Moreover, the formulation of a coherent model has been prevented so far by other uncertainties such as, e.g., the nature of the deep-seated toroidal magnetic field, the $\alpha$-effect and the forbidding range of length scales for the magnetic field and the flow.

A large set of prediction models for solar cycles has been set up based on climatology (past effects), dynamo models, spectral analysis, neural networks, geomagnetic and solar precursor methods. When applied to the prediction of Cycle 24, they resulted in quite differing outcomes ranging from very low to very high maximum values. A critical analysis points out open issues like the difference in predictions from different dynamo models, the possible presence of a memory in the Sun as a physical system for some time span, the maximum time span of far ahead predictions, the possibility to make predictions; when using a mean-field dynamo, this can be ascribed to the lack of physical understanding of the dynamo and to its non-linear chaotic nature. Moreover, the sunspot number is not an adequate indicator at low solar magnetic activity and there is a lack of a precise definitions of solar magnetic activity.

Solar active regions are observed to be formed by the emergence of several small intense flux tubes. Models for the emergence of ephemeral regions are based on MHD-simulation of emerging magnetic flux tubes and statistical and simulated analysis of their emergence for what concerns the total balance of magnetic flux on the solar surface. Anyway, it is still impossible to forecast if the emergence of flux tubes will cause the formation of a fully evolved and recurrent active region or it will give rise to the formation of a structure which will disappear after a short time. High-resolution observations have given new insights on the knowledge of physical phenomena occurring during this phase and might help to solve the above problem. A basic step towards a sunspot predictive system is the automatic classification of sunspot groups from observations based on different approaches like expert systems and artificial neural networks, but further advancements in the knowledge about the physics of sunspot formation and evolution are needed to provide the background for setting up a reliable predictive system.

Theoretical models of solar flares are based on the evidence that they are triggered by an instability of the magnetic field which evolves into a more stable state by changing and reconnecting its topology. 2, 2.5 and 3D models of flares are based on different initial magnetic topologies, main driver and its location, nature of the reconnection process. The first prediction models consisted of expert systems based on sunspot classification and the associated flare productivity. Recent models use artificial neural networks fed by e.g. magnetic gradient data, solar sub-surface flows (curl and divergence of the flow field) derived from local-area helioseismic analyses. Recently proposed, a hybrid system composed of two neural networks can provide both the flaring probability of each sunspot group and the relevant flare intensity probability with a success rate for the final flare prediction around $70 \%$. Other models are based on flare waiting-time or photospheric magnetic features statistics, or use soft X-rays data to predict the evolution of the flare irradiance by providing flare rise, timing 
and magnitude of the peak fluxes, decay to half maximum, termination at background levels and a quantitative flare categorization.

Coronal holes features have a strong influence on the time variability of the heliospheric weather and climate, and are relevant to the physics of cosmic rays. An improved understanding of their role deserves an in-depth analysis of the interplay between $\mathrm{CHs}$ and sunspot/active regions/filament eruptions/CMEs. Near short-term models are aimed to understand their intrinsic nature and climatological models should provide an extended view of what to expect over time in terms of coronal hole characteristic parameters (percentage of corona covered, hole categories, latitudinal/longitudinal distribution, time evolution, periodicities, relationship with other solar activity phenomena, macro and micro-effects in the solar system, etc.), but they are still under development.

Fluid and kinetic models of the solar wind have been developed. Kinetic models are now able to obtain supersonic solar wind speeds in the interplanetary space. As far as densities and temperatures are concerned kinetic models are now in better agreement with the observations than fluid models. A time-dependent 3D MHD model of the heliosphere solves equations for plasma mass, momentum and energy density, and magnetic field. Another model provides the non-monotonic total potential for the protons, with a Lorentzian velocity distribution function for the coronal holes. A third one consists of an MHD model of the solar corona in the range 1-30 solar radii, giving as output the solar coronal temperature, plasma density, pressure velocity and magnetic fields.

XUV-EUV solar irradiance models, based on one or a few indices and that run rapidly, are the best that can be done for space weather operations at this time. None of the indices is representative of the variability of the EUV spectrum at all wavelengths. Models that use additional inputs, such as traditional proxies, irradiance in several bands, and soft X-ray data to reach better accuracy can potentially model both eruptive events and long-term effects, and are therefore suitable for real-time space weather operations.

Despite the variety of scientific models for the coronal mass ejection evolution, no model currently exists that can accurately predict the onset of CMEs at the Sun. The tracking of CMEs from Sun to Earth by the NASA STEREO mission can allow one to prove or reject existing theories on CME initiation, 3D structure, evolution and propagation. A significant step forward towards the development of a reliable automated CME prediction system consists of a machine learning system that analyses the CME-flare associations and predicts, from the input of a flare's properties, if the flare is likely to initiate a CME, flare events from the NGCD flares catalogue and CME events from the SOHO/LASCO CME catalogue are then used to associate $\mathrm{X}$ - and M-class flares with CMEs based on timing information; this information is then fed to the machine learning system to create a set of rules to be used for the automated prediction of CMEs.

Reliable prediction techniques for the radio effectivity of Solar Radio Bursts do not exist. Only nowcasting can be successfully provided based on real-time solar radio measurements. The knowledge on SRB triggering and evolution as well as on possible precursors is fragmentary, so that the only viable approach in setting up a prediction system should be based on a combination of artificial neural networks and/or expert systems.

The characteristics and outcomes of the considered scientific and prediction models for solar weather drivers indicate that they only partially cope with the complex nature of solar activity for the lack of a detailed knowledge of the underlying physics, i.e., in particular, of: (a) the stochastic and chaotic character of the nonlinearly-coupled plasma processes; (b) the plasma processes occurrence at different temporal, spatial and energy scales; (c) the timespace-energy coupling among concurrent physical processes; (d) the fine/hyperfine spatial structure; (e) the fast/ultrafast time evolution. 
This is indicated by the fact that, on one hand, scientific models based on chaos theory and non-linear dynamics reproduce better the observed features, and, on the other hand, that prediction models based on statistics and artificial neural networks perform better.

To date, the solar weather prediction success at most time and spatial scales is far from being satisfactory, but the forthcoming ground- and space-based high-resolution observations can add fundamental tiles to the modelling and predicting frameworks as well as the application of advanced mathematical approaches in the analysis of diachronic solar observations, which are a must to provide comprehensive and homogeneous data sets.

Acknowledgements This review paper has been carried out in the framework of the activities of EC COST Action 724 "Developing the basis for monitoring, modelling and predicting space weather"-Working Group 1 "Monitoring and predicting solar activity for space weather" (2003-2007). The financial support from COST is gratefully acknowledged as well as many fruitful discussions on the considered topics with the WG 1-3 Members. The authors thank the International Space Science Institute (Bern, Switzerland) for the invaluable support during the preparatory phase of this work. M.M., F.Z. and M.S. acknowledge the financial support from the Italian Space Agency (ASI) under the Project "Studies for the Exploration of the Solar System"; F.Z. also the support from the European Commission through the SOLAIRE Network (MRTN-CT-2006035484). V.B. acknowledges the support of the project Stereo/Corona by the German Bundesministerium für Bildung und Forschung through the deutsche Zentrum für Luft- und Raumfahrt e.V. (DLR, German Space Agency) as a collaborative effort with the Max-Planck-Institut für Sonnensystemforschung (MPS) under grant 50 0C 0904. Stereo/Corona is a science and hardware contribution to the optical image package SECCHI, developed for the NASA STEREO mission. H.L. acknowledges the financial support from the Swedish National Space Board under the project "Solar Magnetic Activity: Explored with SOHO/Hinode/SDO". The research by V.B. leading to presented results has received funding from the European Community's Seventh Framework Programme (FP7/2007-2013) under the grant agreement no. 218816 (SOTERIA project; http://www.soteria.eu/). We gratefully acknowledge two anonymous referees, whose comments significantly contributed to improve the quality of the paper, as well as Mrs. S. Fabrizio (INAF-OATS) for the careful proofreading.

\section{References}

E.L. Afraimovich, G.A. Zherebtsov, G.Ya. Smolkov, Dokl. Earth Sci. 417, 1231 (2007). doi:10.1134/ S1028334X07080223

E.L. Afraimovich, V.V. Demyanov, A.B. Ishin, G.Ya. Smolkov, J. Atm. Sol. Terr. Phys. 70, 1985 (2008). doi:10.1016/j.jastp.2008.09.008

T. Amari, J.F. Luciani, Z. Mikić, J. Linker, Astrophys. J. 529, L49 (2000)

T. Amari, T.Z. Boulmezaoud, J.J. Aly, Astron. Astrophys. 446(2), 691 (2006).

S.K. Antiochos, Astrophys. J. 502, L181 (1998)

S.K. Antiochos, C.R. DeVore, J.A. Klimchuk, Astrophys. J. 510, 485 (1999)

C.N. Arge, V.J. Pizzo, J. Geophys. Res. 105, 10465 (2000)

M.J. Aschwanden, Physics of the Solar Corona (Springer, Berlin, 2004)

M.J. Aschwanden et al., Space Sci. Rev. 136, 565 (2008)

G.D.R. Attrill, L.K. Harra, L. van Driel-Gesztelyi, P. Démoulin, Astrophys. J. 656, L101 (2007)

O.G. Badalyan, V. Obridko, N.J. Sykora, Sol. Phys. 199, 421 (2001)

B. Bala, L.J. Lanzerotti, D.E. Gary, D.J. Thomson, Radio Sci. 37, 1018 (2002). doi:10.1029/2001RS002481

G. Barnes, K.D. Leka, E.A. Schumer, D.J. Della-Rose, Space Weather 5, S09002 (2007). doi:10.1029/ 2007SW000317

T.S. Bastian, Adv. Space Res. 32(12), 2705 (2003). doi:10.1016/S0273-1177(03)90454-6

T.S. Bastian, A.O. Benz, D.E. Gary, Ann. Rev. Astron. Astrophys. 36, 131 (1998). doi:10.1146/ annurev.astro.36.1.131

J. Beer, S. Tobias, N. Weiss, Sol. Phys. 181, 237 (1998)

A. Belehaki et al., Space Sci. Rev. (2009, this issue)

B. Bell, G. Noci, Bull. Am. Astron. Soc. 5, 269 (1973)

P.N. Bernasconi, D.M. Rust, M.K. Georgoulis, B.J. La Bonte, Sol. Phys. 209, 119 (2002)

L. Biermann, Z. Astrophys. 29, 274 (1951)

L. Biermann, Mem. Soc. R. Sci. Liege, in-4 13, 291 (1953)

I.A. Bilenko, Astron. Astrophys. 396, 657 (2002) 
I.A. Bilenko, Sol. Phys. 221, 261 (2004)

E.G. Blackman, A. Brandenburg, Astrophys. J. 584, L99 (2003)

J.D. Bohlin, N.R. Sheeley Jr., Sol. Phys. 56, 125 (1978)

A. Bonetti, H.S. Bridge, A.J. Lazarus, E.F. Lyon, B. Rossi, F. Scherb, J. Geophys. Res. 68, 4017 (1963)

V. Bothmer, in Proc. ISCS 2003 Symposium, 'Solar Variability as an Input to the Earth's Environment', ESA, vol. SP-535, (2003), p. 419

V. Bothmer, D.M. Rust, AGU Geophys. Monogr. 99, 139 (1997)

V. Bothmer, R. Schwenn, Adv. Space Res. 17, 319 (1996)

V. Bothmer, R. Schwenn, Ann. Geophys. 16, 1 (1998)

V. Bothmer, D. Tripathi, in Modern Solar Facilities - Advanced Solar Science, ed. by F. Kneer, K.G. Puschmann, A.D. Wittmann (Universitätsverlag Göttingen, Göttingen, 2007), p. 257

V. Bothmer, A. Zhukov, in Space Weather - Physics and Effects, ed. by V. Bothmer, Y. Daglis (Springer/Praxis, Berlin, 2006), p. 31

J.C. Brandt, J.P. Cassinelli, Icarus 5, 47 (1966)

S. Bravo, J.A.L. Cruz-Abeyro, D. Rojas, Ann. Geophys. 16, 49 (1998a)

S. Bravo, G.A. Steward, X. Blanco-Cano, Sol. Phys. 179, 223 (1998b)

G.E. Brückner, R.A. Howard, M.J. Koomen, C.M. Korendyke, D.J. Michels, J.D. Moses, D.G. Socker, K.P. Dere, P.L. Lamy, A. Llebaria, M.V. Bout, R. Schwenn, G.M. Simnett, D.K. Bedford, C.J. Eyles, Sol. Phys. 162, 357 (1995)

L.F. Burlaga, L. Klein, N.R. Sheeley Jr., D.J. Michels, R.A. Howard, M.J. Koomen, R. Schwenn, H. Rosenbauer, J. Geophys. Res. 9, 1317 (1982)

P.J. Bushby, S.M. Tobias, Astrophys. J. 661(2), 1289 (2007). doi:10.1086/516628

R. Cameron, M. Schussler, Astrophys. J. 659, 801 (2007)

P.J. Cargill, Adv. Space Res. 144, 413 (2009). doi:10.1007/s11214-008-9446-9

H. Carmichael, in Proc. of the AAS-NASA Symposium - The Physics of Solar Flares, ed. by W.N. Hess. Washington, National Aeronautics and Space Administration, Science and Technical Information Division (1964), p. 451

A.P. Cerruti, P.M. Kintner, D.E. Gary, L.J. Lanzerotti, E.R. de Paula, H.B. Vo, Space Weather 4, S10006 (2006). doi:10.1029/2006SW000254

J.W. Chamberlain, Astrophys. J. 131, 47 (1960)

P.C. Chamberlin, T.N. Woods, F.G. Eparvier, in 36th COSPAR Scientific Assembly, vol. 36 of COSPAR, Plenary Meeting (2006), p. 395

S. Chapman, Smithsonian Contrib. Astrophys. 2, 1 (1957)

P. Charbonneau, Living Rev. Sol. Phys. 2 (2005). http://www.livingreviews.org/lrsp-2005-2

J. Chela-Flores, G. Jerse, M. Messerotti, C. Tuniz, in From Fossils to Astrobiology Records of Life on Earth and Search for Extraterrestrial Biosignatures, ed. by J. Seckbach, M. Walsh, Cellular Origin, Life in Extreme Habitats and Astrobiology, 12(3), 389 (2008). doi:10.1007/978-1-4020-8837-7_19

F. Chen, Introduction to Plasma Physics (Plenum, New York, 1974)

J. Chen, J. Geophys. Res. 101, 27499 (1996)

J. Chen, J. Krall, J. Geophys. Res. 108, A11, SSH 2-1, (2003). doi:10.1029/2003JA009849

Z. Chen, Y. Gao, Z. Liu, Radio Sci. 40, RS3012 (2005). doi:10.1029/2004RS003066

M.C.M. Cheung, M. Schüssler, F. Moreno-Insertis, Astron. Astrophys. 467, 703 (2007)

P. Chopra, R.S. Dabas, in Proc. 36th COSPAR Scientific Assembly, vol. 36 (2006), p. 909

A.R. Choudhuri, P. Chatterjee, J. Jiang, Phys. Rev. Lett. 98, 131103 (2007)

M.A. Clilverd, E. Clarke, T. Ulich, H. Rishbeth, M.J. Jarvis, Space Weather 4, S09005 (2006). doi:10.1029/ 2005SW000207

T. Colak, R.S. Qahwaji, Sol. Phys. 248(2), 277 (2008). doi:10.1007/s11207-007-9094-3

T. Colak, R.S. Qahwaji, Space Weather (2009). doi:10.1029/2008SW000401

L. Contarino, F. Zuccarello, P. Romano, D. Spadaro, S.L. Guglielmino, V. Battiato, Acta Geophys. 57, 1, 52 (2009). doi:10.2478/s11600-008-0067-1

H. Cremades, V. Bothmer, Astron. Astrophys. 422, 307 (2004)

N. Crooker, J.A. Joselyin, J. Feynman (eds.), Coronal Mass Ejections. AGU Geophysical Monograph, vol. 99, (1997), p. 17

R.S. Dabas, K. Sharma, R.M. Das, K.G.M. Pillai, P. Chopra, N.K. Sethi, Sol. Phys. 250(1), 171 (2008). doi:10.1007/s11207-008-9200-1

T.K. Das, T.N. Chatterjee, A.K. Sen, Sol. Phys. 148, 61 (1993)

J.A. Davies, R.A. Harrison, A.P. Rouillard, N.R. Sheeley Jr., C.H. Perry, D. Bewsher, C.J. Davis, C.J. Eyles, S.R. Crothers, D.S. Brown, Geophys. Res. Lett. 36, L02102 (2009). doi:10.1029/2008GL036182

F. de Meyer, Sol. Phys. 217, 349 (2003)

M. Dikpati, in The Solar-B Mission and the Forefront of Solar Physics. ASP Conf. Ser., vol. 325 (2004), p. 37

M. Dikpati, G. de Toma, P.A. Gilman, C.N. Arge, O.R. White, Astrophys. J. 601, 1136 (2004) 
M. Dikpati, G. de Toma, P.A. Gilman, Geophys. Res. Lett. 33, L05102 (2006)

V. Domingo et al., Space Sci. Rev. 145, 337 (2009). doi:10.1007/s11214-009-9562-1

S.B.F. Dorch, in ESA SP-505: SOLMAG 2002, ed. by H. Sawaya-Lacoste. Magnetic Coupling of the Solar Atmosphere Euroconference Proc. (2002), p. 129

H. Dreicer, Phys. Rev. 115(2), 238 (1959)

H. Dreicer, Phys. Rev. 117(2), 329 (1960)

M. Dryer, S.T. Wu, R.S. Steinolfson, R.M. Wilson, Astrophys. J. 227, 1059 (1979)

Z. Du, S. Du, Sol. Phys. 238, 431 (2006)

T. Dudok de Wit, J. Lilensten, J. Aboudarham, P.-O. Amblard, M. Kretzschmar, Ann. Geophys. 23, 3055 (2005)

T. Dudok de Wit, M. Kretzschmar, J. Aboudarham, P.-O. Amblard, F. Auchère, J. Lilensten, Adv. Space Res. 42, 5, 903 (2008). doi:10.1016/j.asr.2007.04.019

S. Duhau, Sol. Phys. 213, 203 (2003)

G.A. Dulk, Ann. Rev. Astron. Astrophys. 23, 169 (1985)

E. Echer, N.R. Rigozo, D.J.R. Nordemann, L.E.A. Vieira, Ann. Geophys. 22, 2239 (2004). doi:1432-0576/ ag/2004-22-2239

H.C. Euler Jr., S.W. Smith, September 2006 report (2006). http://sail.msfc.nasa.gov/current_solar_report/ CurRpt.pdf

D. Falconer, R. Moore, A. Gary, Bull. Am. Astron. Soc. 38, 135 (2007)

Y. Fan, Living Rev. Sol. Phys. 1, 1 (2004)

J. Feynman, S.B. Gabriel, Sol. Phys. 127, 303 (2004)

T.G. Forbes, E.R. Priest, Astrophys. J. 446, 377 (1995)

T.G. Forbes, J.A. Linker, J. Chen, C. Cid, J. Kóta, M.A. Lee, G. Mann, Z. Mikić, M.S. Potgieter, J.M. Schmidt, G.L. Siscoe, R. Vainio, S.K. Antiochos, P. Riley, Space Sci. Rev. 123(1-3), 251 (2006)

S.E. Forbush, Phys. Rev. 70, 771 (1946)

P. Fox, P. McIntosh, P.R. Wilson, Sol. Phys. 177, 375 (1998)

T. Fragos, E. Rantsiou, L. Vlahos, Astron. Astrophys. 420, 719 (2004)

S.L. Freeland, B.N. Handy, Sol. Phys. 182(2), 497 (1998)

C.D. Fry, T.R. Detman, M. Dryer, Z. Smith, W. Sun, C.S. Deehr, S.-I. Akasofu, C.-C. Wu, S. McKennaLawlor, J. Atm. Sol. Terr. Phys. 69(1-2), 109 (2007). doi:10.1016/j.jastp.2006.07.024

B. Fryxell et al., Astrophys. J. Suppl. Ser. 131(1), 273 (2000)

J. Fuller, S.E. Gibson, G. de Toma, Y. Fan, Astrophys. J. 678(1), 515 (2008). doi:10.1086/533527

P.T. Gallagher, Y.-J. Moon, H. Wang, Sol. Phys. 209, 171 (2002)

D.E. Gary, C.U. Keller (eds.), in Solar and Space Weather Radiophysics - Current Status and Future Developments. Astrophys. Space Sci. Lib., vol. 314 (Springer, Berlin, 2004)

D.E. Gary, L.J. Lanzerotti, G.M. Nita, D.J. Thomson, in Effects of Space Weather on Technology Infrastructure. NATO Sci. Ser., vol. 176 (Springer, Amsterdam, 2005), p. 203. doi:10.1007/1-4020-2754-0_11

M.K. Georgoulis, D.M. Rust, Astrophys. J. Lett. 661, L109 (2007)

A. Gholipour, C. Lucasa, B.N. Araabia, M. Shafiee, J. Atmos. Sol. Terr. Phys. 67, 595 (2005)

S.E. Gibson, B.C. Low, J. Geophys. Res. 105/A8, 18187 (2000)

H. Goldstein, Solar Wind Five. NASA Conf. Publ. CP-2280, 731 (1983)

J.M. Goodman, in Space Weather \& Telecommunications (Springer, New York, 2005), p. 1

J.T. Gosling, J. Birn, M. Hesse, Geophys. Res. Lett. 22, 869 (1995)

K.I. Gringauz, V.V. Bezrukikh, V.D. Ozerov, R.E. Rybchinskiy, Soviet Phys. 5, 361 (1960) (English transl.)

K.-I. Gringauz, V.V. Bezrukikh, V.D. Ozerov, R.E. Rybchinskiy, Space Res. 2, 539 (1961)

G. Haerendel, Astrophys. J. Suppl. Ser. 90, 765 (1994)

H.J. Hagenaar, C.J. Schrijver, A.M. Title, Astrophys. J. 584, 1107 (2003)

G.E. Hale, S.B. Nicholson, in Magnetic Observations of Sunspots 1917-1924, vol. I (Carnegie Institute of Washington, Washington, 1938), p. 56

R.H. Hamid, A.A. Galal, in Solar Activity and its Magnetic Origin, Proceedings of the 233rd Symposium of the IAU, ed. by V. Bothmer, A.A. Hady (Cambridge Univ. Press, Cambridge, 2006), p. 413

L.K. Harra, A.C. Sterling, Astrophys. J. 561, L215 (2001)

K.L. Harvey, PhD Thesis. Astronomical Institute, Utrecht University (1993)

K.L. Harvey, in Solar Wind Eight. ed. by D. Winterhalter et al. AIP Conf. Proc., vol. 382 (1996), p. 9

K.L. Harvey, F. Recely, Sol. Phys. 211, 31 (2002)

J.W. Harvey, N.R. Sheeley Jr., Space Sci. Rev. 23, 139 (1979)

K.L. Harvey, C. Zwaan, Sol. Phys. 148, 85 (1993)

D.H. Hathaway, Space Sci. Rev. 144, 401 (2009). doi:10.1007/s11214-008-9430-4

D.H. Hathaway, R.M. Wilson, Sol. Phys. 224, 5 (2004)

D.H. Hathaway, R.M. Wilson, Geophys. Res. Lett. 33, L18101 (2006). doi:10.1029/2006GL027053

A. Hewish, G. Woan, Lect. Notes Phys. 444, 173 (1995). doi:10.1007/3-540-59109-5_49 
J. Heyvaerts, E.R. Priest, D.M. Rust, Astrophys. J. 216, 123 (1977)

H.E. Hinteregger, Adv. Space Res. 1, 39 (1981)

H.E. Hinteregger, F. Katsura, Geophys. Res. Lett. 8, 1147 (1981)

H.E. Hinteregger, D.E. Bedo, J.E. Manson, Radio Sci. 8, 349 (1973). doi:10.1029/RS008i004p00349

T. Hirayama, Sol. Phys. 34, 323 (1974)

K.M. Hiremath, Astrophys. Space Sci. 314(1-3), 45 (2008). doi:10.1007/s10509-007-9728-9

S. Hirose, Y. Uchida, S. Uemura, T. Yamaguchi, S.B. Cable, Astrophys. J. 551, 586 (2001)

M.Y. Hofer, M. Storini, Sol. Phys. 207, 1 (2002)

M.Y. Hofer, M. Storini, in Solar Wind Ten, ed. by M. Velli et al. AIP Conf. Proc., vol. 679 (2003), p. 234

M. Horstman, Orbital Debris Q. News 9, 4 (2005)

R.A. Howard, D.J. Michels, N.R. Sheeley, M.J. Koomen, Astrophys. J. 263, L101 (1982)

R.A. Howard et al., Space Sci. Rev. 136, 67 (2008)

R. Howe, J. Christensen-Dalsgaard, F. Hill, R. Komm, J. Schou, M.J. Thompson, Astrophys. J. Lett. 701(2), L87 (2009). doi:10.1088/0004-637X/701/2/L87

D.V. Hoyt, K. Schatten, Sol. Phys. 181, 491 (1998)

H.S. Hudson, Astrophys. J. 663, L45 (2007). doi:10.1086/519923

H.S. Hudson, D. Webb, Soft X-ray signatures of coronal mass ejections, in Coronal Mass Ejections, ed. by N.U. Crooker et al. (American Geophysical Union, 1997), p. 27

A.J. Hundhausen, in The Many Faces of the Sun, A summary of Results from NASA's Solar Maximum Mission, ed. by K.T. Strong et al. (Springer, Berlin, 1999), p. 143

R.N. Ikhsanov, V.G. Ivanov, Sol. Phys. 188, 245 (1999)

J.E. Insley, V. Moore, R.A. Harrison, Sol. Phys. 160, 1 (1995)

B.V. Jackson, D.F. Webb, P.L. Hick, J.L. Nelson, (Phillips Laboratory, Directorate of Geophysics, Hanscom Air Force Base, 01731-3010, USA), Sci. Rep. 4 (1994)

R. Jain, in Proc. 36th COSPAR, vol. 36 (2006), p. 642

J. Javaraiah, Mon. Not. R. Astron. Soc. Lett. 377, L34 (2007). doi:10.1111/j.1745-3933.2007.00298.x

J.M. Jensen, H. Lundstedt, M.J. Thompson, F.P. Pijpers, S.P. Rajaguru, in Proc. of the SOHO 14/Gong 2004 Workshop. ESA, vol. SP-559 (2004), p. 497

J. Jing, H. Song, V. Abramenko, C. Tan, H. Wang, Astrophys. J. 644, 1273 (2006)

K. Jockers, Astron. Astrophys. 6, 219 (1970)

R.P. Kane, Sol. Phys. 189, 217 (1999)

R.P. Kane, Sol. Phys. 243, 205 (2007)

S.L. Keil, T.R. Rimmele, J. Wagner, Earth Moon Planets 104(1-4), 77 (2009). doi:10.1007/ s11038-008-9258-7

J.A. Kennewell, in Proc. International Conference on Antennas and Propagation (ICAP 89) (London, England and Piscataway, NJ, Institution of Electrical Engineers), Part 2 (A90-27776 11-32), p. 334 (1989)

M.N. Khramova, S.A. Krasotkin, E.V. Kononovich, in Proc. of the Second Solar Cycle and Space Weather Euroconference. ed. by H. Sawaya-Lacoste. ESA, vol. SP-477 (2002), p. 229

M.-H.Y. Kim, J.W. Wilson, F.A. Cucinotta, Adv. Space Res. 37, 1741 (2006). doi:10.1016/j.asr.2004.11.036

J.A. Klimchuk, Astrophys. J. 354, 745 (1990)

J.A. Klimchuk, in Space Weather, ed. by P. Song, H. Singer, G. Siscoe. Geophysical Monograph, vol. 125 (Am. Geophys. Union, Washington, 2001), p. 143

J.A. Klobuchar, J.M. Kunches, A.J. VanDierendonck, GPS Solutions 3(2), 69 (1999)

R.A. Kopp, G.W. Pneuman, Sol. Phys. 50, 85 (1976)

J. Krall, J. Chen, R. Santoro, Astrophys. J. 539, 964 (2000)

M. Kretzschmar, J. Lilensten, J. Aboudarham, Astron. Astrophys. 419, 345 (2004). doi:10.1051/ 0004-6361:20040068

M. Kretzschmar, J. Lilensten, J. Aboudarham, Adv. Space Res. 37, 341 (2006). doi:10.1016/ j.asr.2005.02.029

A.S. Krieger, A.F. Timothy, E.C. Roelof, Sol. Phys. 29, 505 (1973)

H. Kunow, N.U. Crooker, J.A. Linker, R. Schwenn, R. von Steiger, Space Sci. Rev. 123, 1-2 (2006). doi:10.1007/s11214-006-9007-z

Y.C. Lai, C. Grebogi, J. Kurths, Phys. Rev. E 59(3), 2907 (1999). doi:10.1103/PhysRevE.59.2907

D. Lamb, Bull. Am. Astron. Soc. 41, 811 (2009)

H. Lamy, V. Pierrard, M. Maksimovic, J.F. Lemaire, J. Geophys. Res. 108, 1047 (2003)

S. Landi, F.G.E. Pantellini, Astron. Astrophys. 372, 686 (2001)

P. Lantos, Sol. Phys. 229, 373 (2006). doi:10.1007/s11207-006-0128-z

L.J. Lanzerotti, in Handbook of the Solar-Terrestrial Environment, ed. by Y. Kamide, A.C.-L. Chian (Springer, Berlin, 2007), p. 424

L.J. Lanzerotti, D.E. Gary, D.J. Thomson, C.J. MacIennan, Bell Labs Tech. J. 7, 159 (2002). doi:10.1002/ bltj30 
L.J. Lanzerotti, D.E. Gary, G.M. Nita, D.J. Thomson, C.G. McIennan, Adv. Space Res. 36(12), 2253 (2005)

C. Lathuillère, M. Menvielle, J. Lilensten, T. Amari, S.M. Radicella, Ann. Geophys. 20, 1081 (2002)

A. Lazarian, E.T. Vishnian, J. Cho, Astrophys. J. 603, 180 (2004)

J.L. Lean, J.M. Picone, J.T. Emmert, J. Geophys. Res. 114(A7), A07301 (2009). doi:10.1029/2009JA014285

J. Lemaire, M. Scherer, J. Geophys. Res. 76, 7479 (1971)

R.P. Lepping, J.A. Jones, L.F. Burlaga, J. Geophys. Res. 95, 11957 (1990)

R.P. Lepping et al., Sol. Phys. 204(1/2), 285 (2001)

J.A. Linker, Z. Mikić, Astrophys. J. 438, L45 (1995)

J.A. Linker, Z. Mikić, R. Lionello, P. Riley, T. Amari, D. Odstrcil, Phys. Plasmas 10(5), 1971 (2003)

B.W. Lites, A. Skumanich, V. Martínez Pillet, Astron. Astrophys. 333, 1053 (1998)

M. Lockwood, R. Stamper, M.N. Wild, Nature 399, 437 (1999)

B.C. Low, Sol. Phys. 167, 217 (1996)

B.C. Low, B. Fong, Y. Fan, Astrophys. J. 594, 1060 (2003)

J.G. Luhmann, Y. Li, C.N. Arge, P.R. Gazis, R. Ulrich, J. Geophys. Res. 107(A8), 1154 (2002). doi:10.1029/2001JA007550

H. Lundstedt, Adv. Space Res. 38, 862 (2006)

H. Lundstedt, Acta Geophys. 57(1), 31 (2009). doi:10.2478/s11600-008-0062-6

H. Lundstedt, L. Liszka, R. Lundin, R. Muscheler, Ann. Geophys. 24, 1 (2006)

B.R. Luo, C.C. Duan, Y. Cao, in Proc. Workshop on Solar Flares and Related Disturbances, ed. by E. Sagawa, M. Akioka (Hiraiso Solar Terrestrial Research Center, Communications Research Laboratory, Ibaraki, 1997), p. 130

R.M. MacQueen, J.A. Eddy, J.T. Gosling, E. Hildner, R.H. Munro, G.A. Newkirk, A.I. Poland, C.L. Ross, Astrophys. J. Lett. 187, L85 (1974)

M. Maksimovic, V. Pierrard, J.F. Lemaire, Astron. Astrophys. 324, 725 (1997)

W. Manchester IV, et al. J. Geophys. Res. 109/A1, A01102 (2004)

A.S. Mandal, P. Raychaudhuri, in Proc. 29th International Cosmic Ray Conference Pune, vol. 9, (2005), p. 123

G. Mann, Astron. Nachr. 326, 618 (2005)

D. Maravilla, A. Lara, J.F. Valdés Galicia, B. Mendoza, Sol. Phys. 203, 27 (2001)

G. Maris, A. Oncica, Sun Geospace, vol. 1 (2006). http://www.stil.bas.bg/IHY/forms/SUN_GEO200601.html

G. Maris, M.D. Popescu, D. Besliu, in Multi-Wavelength Investigations of Solar Activity, ed. by A.V. Stepanov, E.E. Benevolenskaya, A.G. Kosovichev. IAU Symposium, vol. 223 (Cambridge Univ. Press, Cambridge, 2004), p. 127

D.J. McComas, H.A. Elliott, N.A. Schwadron, J.T. Gosling, R.M. Skoug, B.E. Goldstein, Geophys. Res. Lett. 30(10), 24-1 (2003). doi:10.1029/2003GL017136

P.S. McIntosh, Sol. Phys. 125, 251 (1990). doi:10.1007/BF00158405

P.S. McIntosh, in Solar Interior Processes Suggested by Large-Scale Surface Patterns, The Solar Cycle, ed. by K.L. Harvey. ASP Conf. Proc., vol. 27 (1992), p. 14

P.S. McIntosh, R.J. Thompson, E.C. Willock, Nature 360, 322 (1992)

S.W. McIntosh, A.R. Davey, D.M. Hassler, J.D. Armstrong, W. Curdt, K. Wilhelm, G. Lin, Astrophys. J. 654(1), 650 (2007)

M. Messerotti, in The Dynamic Sun, Proc. of the Summerschool and Workshop held at the Solar Observatory Kanzelhöhe, Kärnten, Austria, August 30-September 10, 1999, ed. by A. Hanslmeier, M. Messerotti, A. Veronig. Astrophys. Space Sci. Lib., vol. 259 (Kluwer, Dordrecht, 2001), p. 69

M. Messerotti, in Proc. SOLSPA: The Second Solar Cycle and Space Weather Euroconference. ESA, vol. SP-477 (2002), p. 607

M. Messerotti, in Life in the Universe: From the Miller Experiment to the Search for Life on Other Worlds, ed. by J. Seckbach, J. Chela-Flores, T. Owen, F. Raulin (Springer, Berlin, 2005), p. 177

M. Messerotti, in The Future of Life and the Future of our Civilization, ed. by V. Burdyuzha (Springer, Berlin, 2006), p. 133

M. Messerotti, in Proc. Solar Activity: Exploration, Understanding and Prediction, ed. by H. Lundstedt. ESA CDROM, ESA Publications Division, Nordwijk (2007), p. 11

M. Messerotti, AIP Conf. Proc. vol. 1043 (2008), p. 277. doi:10.1063/1.2993661

M. Messerotti, Earth Moon Planets 104(1-4), 51 (2009). doi:10.1007/s11038-008-9265-8

M. Messerotti, J. Chela-Flores, in Space Weather: Research Towards Applications in Europe 2nd European Space Weather Week, ed. by J. Lilensten. Astrophys. Space Sci. Lib., vol. 344 (Springer, Amsterdam, 2007), p. 49

M. Messerotti, J. Chela-Flores, Acta Geophys. 57(1), 64 (2009). doi:10.2478/s11600-008-0082-2

R. Mewe, E.H.B. Gronenschild, G.H.J. van den Oord, Astron. Astrophys. Suppl. Ser. 62, 197 (1985)

R. Mewe, J.R. Lemen, G.H.J. van den Oord, Astron. Astrophys. Suppl. Ser. 65(3), 511 (1986)

Z. Mikić, J. Linker, Bull. Am. Astron. Soc. 31, 918 (1999) 
R.W. Miller, Lect. Notes Phys. 329, 107 (1989). doi:10.1007/3-540-51044-3_20

J.A. Miller et al., J. Geophys. Res. 10(A7), 14631 (1997)

Y.-J. Moon, G.S. Choe, H.S. Yun, Y.D. Park, J. Geophys. Res. 106(A12), 29951 (2001)

F. Moreno Insertis, T. Emonet, Astrophys. J. 472, L53 (1996)

M.D. Mundt, W.B. II Maguire, R.R.P. Chase, J. Geophys. Res. 96, 1705 (1991). doi:10.1029/90JA02150

P. Navarro-Peralta, A. Sanchez-Ibarra, Sol. Phys. 153, 169 (1994)

W.M. Neupert, V. Pizzo, J. Geophys. Res. 79, 3701 (1974)

G.M. Nita, D.E. Gary, L.J. Lanzerotti, D.J. Thomson, Astrophys. J. 570, 423 (2002). doi:10.1086/339577

G.M. Nita, D.E. Gary, L.J. Lanzerotti, Space Weather 2, S1 1005 (2004). doi:10.1029/2004SW000090

G. Noci, Sol. Phys. 28, 403 (1973)

G. Noci, Plasmas in the Universe, in Proc. International School of Physics 'Enrico Fermi', Course CXLII, Varenna, 6-16 July 1999, ed. by B. Coppi, A. Ferrari (IOS Press, Amsterdam, 2000), p. 227

Å. Nordlund, R.F. Stein, M. Asplund, Living Rev. Sol. Phys. 2 (2009). http://www.livingreviews.org/ lrsp-2009-2

Å. Nordlund et al., Astrophys. J. 392, 647 (1992)

V.N. Obridko, B.D. Shelting, Sol. Phys. 187, 185 (1999)

D. Odstrcil, Adv. Space Res. 32(4), 497 (2003)

M. Ossendrijver, Astron. Astrophys. Rev. 11, 287 (2003)

E.N. Parker, Astrophys. J. 122, 293 (1955)

E.N. Parker, Astrophys. J. 128, 664 (1958)

E.N. Parker, Space Sci. Rev. 144, 15 (2009). doi:10.1007/s11214-008-9445-x

C.E. Parnell, C.E. DeForest, H.J. Hagenaar, B.A. Johnston, D.A. Lamb, B.T. Welsch, Astrophys. J. 698(1), 75 (2009)

C.E. Parnell, C.E. DeForest, H.J. Hagenaar, B.A. Johnston, D.A. Lamb, B.T. Welsch, Astrophys. J. 698, 75 (2009)

S. Patsourakos, A. Vourlidas, Y.M. Wang, G. Stenborg, A. Thernisien, Sol. Phys. (2009). doi:10.1007/ s11207-009-9386-x

J. Pérez-Peraza, in Rayos Cósmicos 98, ed. by J. Medina et al. (Servicio de Publicaciones de la Universidad de Alcalá, Alcalá, 1998), p. 97

W.D. Pesnell, Sol. Phys. 252, 209 (2008). doi:10.1007/s11207-008-9252-2

E.R. Priest, T.G. Forbes, Magnetic Reconnection: MHD Theory and Applications (Cambridge University Press, Cambridge, 2000)

E.R. Priest, T.G. Forbes, Astron. Astrophys. Rev. 10, 313 (2002)

E.R. Priest, C.E. Parnell, S.F. Martin, Astrophys. J. 427, 459 (1994)

R. Qahwaji, T. Colak, Sol. Phys. 241(1), 195 (2007). doi:10.1007/s11207-006-0272-5

R. Qahwaji, T. Colak, M. Al-Omari, S. Ipson, Sol. Phys. 248(2), 471 (2008). doi:10.1007/s11207-007-9108-1

D.M. Rabin, Bull. Am. Astron. Soc. 210, 9205 (2007)

O. Regev, in Chaos and Complexity in Astrophysics (Cambridge University Press, Cambridge, 2006). doi: $10.2277 / 0521855349$

M. Rempel, M. Schüssler, M. Knölker, Astrophys. J. 691(1), 640 (2009). doi:10.1088/0004-637X/691/1/640

P.G. Richards, J.A. Fennelly, D.G. Torr, J. Geophys. Res. 99, 8981 (1994)

P.G. Richards, T.N. Woods, W.K. Peterson, Adv. Space Res. 37, 315 (2006). doi:10.1016/j.asr.2005.06.031

E. Robbrecht, D. Berghmans, Astron. Astrophys 425, 1097 (2004). doi:10.1051/0004-6361:20041302

I.I. Roussev, I.V. Sokolov, T.G. Forbes, T.I. Gombosi, M.A. Lee, J.I. Sakai, Astrophys. J. 605, L73 (2004)

I.I. Roussev et al., Astrophys. J. 595, L57 (2003)

B. Ruiz Cobo, J.C. del Toro Iniesta, Astrophys. J. 398(1), 375 (1992)

C.T. Russell (ed.), in The STEREO Mission. Space Sci. Rev., 1-4 (2008)

D.M. Rust, Space Sci. Rev. 34, 21 (1983)

D.A. Rust, A. Kumar, Sol. Phys. 155, 69 (1994)

A. Sainz-Dalda, L.R. Bellot-Rubio, Astron. Astrophys. 481, L21 (2008). doi:10.1051/0004-6361:20079115

A. Sanchez-Ibarra, Sol. Phys. 125, 125 (1990)

A. Sanchez-Ibarra, M. Barraza-Paredes, Report UAG-102. WDCA, Boulder, U.S.A. (1992)

K.H. Schatten, Geophys. Res. Lett. 32(21), L21106 (2005). doi:10.1029/2005GL024363

R. Schlichenmaier, Space Sci. Rev. 144, 213 (2009). doi:10.1007/s11214-008-9465-6

C.J. Schrijver, C. Zwaan, Solar and Stellar Magnetic Activity (Cambridge University Press, Cambridge, 2000)

C.J. Schrijver, A.M. Title, A.A. van Ballegooijen, H.J. Hagenaar, R.A. Shine, Astrophys. J. 487, 424 (1997)

R.J. Schunk, J.J. Sojka, J.V. Eccles, D. Thompson, AFRL Rep. AFRL-VS-HA-TR-98-0001. Air Force Res. Lab., Hanscom AFB, MA (1997)

R. Schwenn, A. dal Lago, E. Huttunen, W.D. Gonzalez, Ann. Geophys. 23, 1033 (2005) 
D. Shaw, in Proc. Fourth Annual Rocky Mountain Conference on Artificial Intelligence, ed. by J.H. Alexander. Denver, Co. (1989), p. 6

N.R. Sheeley Jr., R.A. Howard, M.J. Koomen, D.J. Michels, R. Schwenn, K.-H. Mühlhäuser, H. Rosenbauer, J. Geophys. Res. 90, 163 (1985)

K. Shibata, in Encyclopedia of Astronomy and Astrophysics, ed. by P. Murdin (Institute of Physics Publishing, Bristol, 2001), p. 11. http://eaa.iop.org/abstract/0333750888/2272

J.J. Sidorowich, in Proc. IEEE Int. Conf. on Acoustics, Speech, and Signal Processing 1992. ICASSP-92, vol. 4, (1992), p. 121. doi:10.1109/ICASSP.1992.226471

G.W. Simon, A.M. Title, N.O. Weiss, Astrophys. J. 561, 427 (2001)

S.K. Solanki, Astron. Astrophys. Rev. 11, 153 (2003)

D. Spadaro, S. Billotta, L. Contarino, P. Romano, F. Zuccarello, Astron. Astrophys. 425, 309 (2004)

E.A. Spiegel, Space Sci. Rev. 144, 25 (2009). doi:10.1007/s11214-008-9470-9

J.C. Sprott, Chaos and Time-Series Analysis (Oxford University Press, Oxford, 2003)

H.C. Spruit, Astron. Astrophys. 98, 155 (1981)

O.C. St. Cyr, J.T. Burkepile, A.J. Hundhausen, A.R. Lecinski, J. Geophys. Res. 104, 12493 (1999)

O.C. St. Cyr, R.A. Howard, N.R. Sheeley Jr., S.P. Plunkett, D.J. Michels, S.E. Paswaters, M.J. Koomen, G.M. Simnett, B.J. Thompson, J.B. Gurman, R. Schwenn, D.F. Webb, E. Hildner, P.L. Lamy, J. Geophys. Res. 105, 18169 (2000)

A.C. Sterling, H.S. Hudson, B.J. Thompson, D.M. Zarro, Astrophys. J. 532, 628 (2000)

J.M. Stone, M.L. Norman, Astrophys. J. Suppl. Ser. 80(2), 753 (1992)

M. Storini, M.Y. Hofer, in 9th European Meeting on Solar Physics: Magnetic Fields and Solar Processes, ed. by A. Wilson. ESA, vol. SP-448 (1999), p. 889

M. Storini, M.Y. Hofer, Geophys. Res. Abstracts 5, 08529 (2003a)

M. Storini, M.Y. Hofer, Report CNR/IFSI-2003-14. Istituto di Fisica dello Spazio Interplanetario, Roma, (2003b)

M. Storini, M.Y. Hofer, J. Sykora, Adv. Space Res. 38, 912 (2006)

P.A. Sturrock, Nature 211, 695 (1966)

L. Svalgaard, E.W. Cliver, Y. Kamide, Geophys. Res. Lett. 32, L01104 (2005)

K. Tapping, in Encyclopedia of Astronomy and Astrophysics, ed. by P. Murdin (Institute of Physics Publishing, Bristol, 2000), p. 2047

T. Tél, M. Gruiz, Chaotic Dynamics: An Introduction Based on Classical Mechanics (Cambridge University Press, Cambridge, 2006)

M. Ternullo, L. Contarino, P. Romano, F. Zuccarello, Astronom. Nachr. 327, 36 (2006)

J. Thalmann, T. Wiegelmann, Astron. Astrophys. 484, 2, 495 (2008)

A.F.R. Thernisien, R.A. Howard, A. Vourlidas, Astrophys. J. 652, 763 (2006)

R.J. Thompson, Sol. Phys. 148, 383 (1993)

B.J. Thompson, S.P. Plunkett, J.B. Gurman, J.S. Newmark, O.C. St. Cyr, D.J. Michels, J.-P. Delaboudinière, Geophys. Res. Lett. 25, 2461 (1998)

V.S. Titov, P. Démoulin, Astron. Astrophys. 351, 707 (1999)

S.M. Tobias, R. Soc. Lond. Philos. Tr. A 360, 2741 (2002)

S. Tobias, N.O. Weiss, V. Kirk, Mon. Not. R. Astron. Soc. 273(4), 1150 (1995)

W.K. Tobiska, J. Atmos. Terr. Phys. 53, 1005 (1991)

W.K. Tobiska, J. Geophys. Res. 98, 18, 879 (1993)

W.K. Tobiska, American Institute of Aeronautics and Astronautics, 0069, 1 (2005)

W.K. Tobiska, American Institute of Aeronautics and Astronautics 0453, 1 (2008)

W.K. Tobiska, S. Bouwer, in 2004 AGU Fall Meeting (2004), Abstract no. SA43B-01

W.K. Tobiska, S. Bouwer, Adv. Space Res. 37, 347 (2006)

W.K. Tobiska, S.D. Bouwer, in 2005 Ionospheric Effects Symposium. ed. by J.M. Goodman. JMG Associates (2005), p. 76

W.K. Tobiska, F.G. Eparvier, Sol. Phys. 177, 147 (1998)

W.K. Tobiska, T. Woods, F. Eparvier, R. Viereck, L. Floyd, D. Bouwer, G. Rottman, O.R. White, J. Atmos. Terr. Phys. 62, 1233 (2000)

T. Török, B. Kliem, V.S. Titov, Astron. Astrophys. 413, L27 (2003)

M.R. Torr, D.J. Torr, Geophys. Res. Lett. 6, 771 (1979)

M.R. Torr, D.J. Torr, J. Geophys. Res. 90, 6675 (1985)

Toth, et al., J. Geophys. Res. 110, A12226 (2005)

R. Tousey, Adv. Space Res. 13, 713 (1973)

D.K. Tripathi, V. Bothmer, H. Cremades, Astron. Astrophys. 422(1), 337 (2004)

V. Tritakis, H. Mavromichalaki, G. Giouvanellis, in Recent Advances in Astronomy and Astrophysics: 7 th International Conference of the Hellenic Astronomical Society, ed. by N. Solomos. AIP Conf. Proc., vol. 848 (2006), p. 154. doi:10.1063/1.2347972 
L.B. Tsirulnik, T.V. Kuznetsova, V.N. Oraevsky, Adv. Space Res. 20, 2369 (1997). doi:10.1016/ S0273-1177(97)00909-5

S. Tsuneta, Astrophys. J. 483, 507 (1997)

I.G. Usoskin, S.K. Solanki, G.A. Kovaltsov, Astron. Astrophys. 471(1), 301 (2007). doi:10.1051/ 0004-6361:20077704

R. Vainio, et al., Space Sci. Rev. (2009, this issue)

L. van Driel-Gesztelyi, in ESA SP-505: SOLMAG 2002, ed. by H. Sawaya-Lacoste. Magnetic Coupling of the Solar Atmosphere Euroconference Proc. (2002), p. 113

A. Vourlidas, D. Buzasi, R.A. Howard, E. Esfandiari, in ESA SP-506: Solar Variability: From Core to Outer Frontiers (2002), p. 91

M. Waldmeier, Sol. Phys. 70, 251 (1981)

Y.-M. Wang, S.H. Hawley, N.R. Sheeley Jr., Science 271(5248), 464 (1996). doi:10.1126/science. 271.5248 .464

J.-L. Wang, J.-C. Gong, S.-Q. Liu, G.-M. Le, J.-L. Sun, Chin. J. Astron. Astrophys. 2, 557 (2002)

H.P. Warren, Adv. Space Res. 37, 359 (2006) doi:10.1016/j.asr.2005.10.028

H.P. Warren, J.T. Mariska, J. Lean, J. Geophys. Res. 103, 12077 (1998)

D.F. Webb, B.V. Jackson, J. Geophys. Res. 95, 20641 (1990)

D.F. Webb, J.M. Davis, P.S. McIntosh, Sol. Phys. 92, 109 (1984)

D.F. Webb, B.V. Jackson, P. Hick, R. Schwenn, V. Bothmer, D. Reames, Adv. Space Res. 13(9), 971 (1993)

D.F. Webb, E.W. Cliver, N.U. Crooker, O.C. St. Cyr, B.J. Thompson, J. Geophys. Res. 105(A4), 7491 (2000)

D.F. Webb, et al., J. Geophys. Res. 111, A12101 (2006)

N.O. Weiss, M.J. Thompson, Space Sci. Rev. 144, 53 (2009). doi:10.1007/s11214-008-9435-Z

M.S. Wheatland, Sol. Phys. 203, 87 (2001)

M.S. Wheatland, Space Weather 3, S07003 (2005). doi:10.1029/2004SW000131

M.J. Wills-Davey, C.E. DeForest, J.O. Stenflo, Astrophys. J. 664, 556 (2007)

T.N. Woods, F.G. Eparvier, S.M. Bailey, P.C. Chamberlin, J. Lean, G.J. Rottman, S.C. Solomon, W.K. Tobiska, D.L. Woodraska, J. Geophys. Res. 110, 1312 (2005)

J.W. Woolley, P.K. Agarwal, J. Baker, Int. J. Num. Methods Fluids (2009). doi:10.1002/fld.2117

S.T. Wu, Space Sci. Rev. 32, 115 (1982)

Xie et al., J. Geophys. Res. 109, A03109 (2004). doi:10.1029/2003JA010226

T. Xu, J. Wu, Z.-S. Wu, Q. Li, Chin. J. Astron. Astrophys. 8, 337 (2008)

Y. Yatsuyanagi, T. Hatori, T. Kato, Progr. Theor. Phys., Suppl. 138, 714 (2000)

D.M. Zarro, A.C. Sterling, B.J. Thompson, H.S. Hudson, N. Nitta, Astrophys. J. 520, L139 (1999)

J. Zhang, Astrophys. J. 559, 452 (2001)

U. Ziegler, Astron. Astrophys. 435(2), 385 (2005)

J.B. Zirker (ed.), Coronal Holes and High Speed Wind Streams (Colorado Associated University Press, Boulder, 1977)

F. Zuccarello, V. Battiato, L. Contarino, P. Romano, D. Spadaro, L. Vlahos, Astron. Astrophys. 442, 661 (2005)

C. Zwaan, Sol. Phys. 100, 397 (1985) 